Canadian Journal of Fisheries and Aquatic Sciences

Canadian

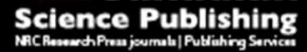

Journal canadien des sciences halieutiques et aquatiques

\title{
Examining non-stationarity in the recruitment dynamics of fishes using Bayesian change point analysis
}

\begin{tabular}{|c|c|}
\hline Journal: & Canadian Journal of Fisheries and Aquatic Sciences \\
\hline Manuscript ID & cjfas-2016-0177.R2 \\
\hline Manuscript Type: & Article \\
\hline Date Submitted by the Author: & 13-Sep-2016 \\
\hline Complete List of Authors: & $\begin{array}{l}\text { Perälä, Tommi; University of Helsinki, Department of Environmental } \\
\text { Sciences } \\
\text { Swain, Douglas; Department of Fisheries and Oceans, } \\
\text { Kuparinen, Anna; University of Helsinki, }\end{array}$ \\
\hline Keyword: & $\begin{array}{l}\text { change point, regime shift, ricker model, beverton-holt model, sequential } \\
\text { monte carlo }\end{array}$ \\
\hline
\end{tabular}




\section{Examining non-stationarity in the recruitment}

\section{2 dynamics of fishes using Bayesian change point}

3 analysis

4

5 Tommi A. Perälä ${ }^{*}$, Douglas P. Swain ${ }^{2}$, Anna Kuparinen ${ }^{1}$,

6

$7{ }^{1}$ Department of Environmental Sciences, P. O. Box 65, 00014 University of Helsinki,

$8 \quad$ Finland

$9{ }^{2}$ Department of Fisheries and Oceans Canada, Gulf Fisheries Centre, P.O. Box 5030,

10 Moncton, NB E1C 9B6, Canada

$11 *$ Corresponding author: tommi.perala@helsinki.fi

12 


\section{Abstract}

14 Marine ecosystems can undergo regime shifts, which result in non-stationarity in the

15 dynamics of the fish populations inhabiting them. The assumption of time-invariant

16 parameters in stock-recruitment models can lead to severe errors when forecasting renewal

17 ability of stocks that experience shifts in their recruitment dynamics. We present a novel

18 method for fitting stock-recruitment models using the Bayesian online change point

19 detection algorithm, which is able to cope with sudden changes in the model parameters.

20 We validate our method using simulations, and apply it to empirical data of four demersal

21 fishes in the southern Gulf of St. Lawrence. We show that all of the stocks have

22 experienced shifts in their recruitment dynamics that cannot be captured by a model which

23 assumes time-invariant parameters. The detected shifts in the recruitment dynamics result

24 in clearly different parameter distributions and recruitment predictions between the

25 regimes. The present study illustrates how stock-recruitment relationships can experience

26 shifts which, if not accounted for, can lead to false predictions about a stock's recovery

27 ability and resilience to fishing.

29 Keywords: Change point, Productivity, Regime shift, Reproduction, Ricker model,

30 Beverton-Holt model, Spawning stock, Sequential Monte Carlo 


\section{1. Introduction}

32 Reproduction forms a vital link between current and future generations. The sustainability

33 of fishing is largely attributable to the ability of a fish stock to renew itself such that it

34 maintains its abundance at a target level despite biomass removal by fishing (Hilborn

35 2005). The study of fish stock reproduction has largely focused on the relationship

36 between the reproducing component of the stock (i.e. the spawning stock) and the

37 recruitment (Hilborn and Walters 1992). Recruitment of a fish stock is typically predicted

38 using a curve that links spawning-stock biomass with recruit abundance, that is, the

39 stock-recruitment (S-R) relationship. Traditional formulations of this relationship are

40 provided by Ricker (Ricker 1954) and Beverton-Holt (Beverton and Holt 1957) S-R

41 models (but for a comprehensive list of models, see, e.g., Needle 2001). A characteristic of

42 both these models is that at low spawning stock levels recruitment grows steeply as a

43 function of spawning stock biomass, but the rate of increase reduces as spawning stock

44 biomass increases (Hilborn and Walters 1992).

46 Although S-R relationships form one of the cornerstones for fisheries stock assessments

47 and are widely used for projecting stock development under alternative fishing scenarios,

48 their applicability remains widely debated. Unexplained variations in recruitment have

49 long been known to be present in population dynamics (Hjort 1914). A recent meta-study

50 illustrates this by showing that recruitment and spawning stock biomass show no

51 correlation for $61 \%$ of 224 stocks recorded in the RAM legacy database (Szuwalski et al.

52 2014). In general, fish populations show great variability around the mean recruitment

53 predicted by S-R models, and the resulting large uncertainty in the estimates limits the

54 utility of these predictions. Variations in temperature, food availability, and predation 
55 pressure have been identified as key drivers of such environment-driven recruitment

56 variability (e.g. Brunel and Boucher 2007; Fauchald 2010; Olsen et al. 2011; Okamoto et

57 al. 2012), and it has been suggested that for some species environmental drivers might be a

58 more important source of recruitment variability than the spawning stock biomass

59 (Szuwalski et al. 2014). For example, in the North Sea about 45\% of Atlantic cod (Gadus

60 morhua) recruitment variability was found to be associated with temperature and food

61 availability, whereas only $10 \%$ of variability was attributable to spawning stock biomass

62 (Olsen et al. 2011). In principle, as long as environmental correlates can be identified, S-R

63 models can be modified to account for the additional sources of variation (for examples to

64 this end, see Hilborn and Walters 1992, Chen and Irvine 2001, Begg and Marteinsdottir

65 2002, Ciannelli et al. 2004, Stige et al. 2013). However, such approaches make the implicit

66 assumption that future recruitment can be predicted by observations made in the past, i.e.

67 that correlations established in the past are indicative of correlations in the future. If fish

68 population dynamics or the entire ecosystem shifts from one state into another such that

69 key productivity parameters and their covariates change, S-R models fitted to observations

70 before such a shift may not describe recruitment after the shift even if the correct

71 environmental correlates have been identified.

73 It has been increasingly recognized that marine ecosystems can undergo very rapid shifts

74 from one relatively stable state to another due to internal or external forcing (deYoung et

75 al. 2008, Vasilakopous and Marshall 2015). The time periods where the ecosystem stays in

76 a certain relatively stable state are often denoted as 'regimes', and the change from one

77 stable state to another is called a 'regime shift'. In fish stocks, the regime shifts may result

78 in changes in their population dynamics. For example, in the 1980 s, regime shifts in the 
79 North Sea and the Baltic Sea were followed by a decline in the recruitment of Atlantic cod

80 (Gadus morhua) (Beaugrand 2004, Alheit et al. 2005). It has also been shown using

81 surplus production models that shifts in marine fish stock productivity are very common.

82 Of the 230 fish stocks studied by Vert-pre et al. (2013), 69\% were best explained by a

83 model that took shifts in productivity into account.

84

85 In light of the observed regime shifts in fish stocks and their ecosystems, it seems likely

86 that the parameters of the S-R relationships can also experience shifts. The times when the

87 parameters change are often called change points. Such shifts may partially explain the

lack of correlation between spawning stock biomass and recruitment. Indeed, using

89 Bayesian inference, Munch and Kottas (2009) showed that the recruitment dynamics of

90 Japanese sardine (Sardinops melanostictus) is characterized by shifts between two

91 alternative regimes in Ricker model parameters. If a fish stock experiences notable shifts

92 in its recruitment dynamics, fitting S-R models using all available data may result in biases

93 in the mean recruitment and unnecessarily large estimates of uncertainty. In 27 of 38 North

94 Atlantic fish stocks studied using maximum-likelihood methods, the relationship between

95 recruitment and spawning stock biomass was not constant but instead was better explained

96 by a model allowing for a stepwise change in the S-R relationship (Ottersen et al. 2013).

97 While showing that S-R relationships can indeed show shifts, the currently applied

98 modelling approaches are limited in the sense of either considering only two alternative

99 regimes or by omitting uncertainty about the inferred timings of the shifts.

100

101 The objective of the present study is to challenge the commonly made assumption that the

102 parameters in S-R models are time-invariant and to investigate whether S-R dynamics 
103 show signals of regime shifts. To this end, we utilize the Bayesian online change point

104 detection algorithm (BOCPD; Adams and MacKay 2007, Perälä and Kuparinen 2015) and

105 expand the methodology to study shifts in the parameters of the Ricker and Beverton-Holt

106 S-R models. Previously, BOCPD has been used to infer change points in linear models

107 with conjugate priors. Here we show how to implement non-linear models with arbitrary

108 prior distributions within a BOCPD framework using Sequential Monte Carlo (SMC)

109 methods. To our knowledge, this has not been done before. We validate our method using

110 simulations, and explore the S-R dynamics of Atlantic cod (Gadus morhua), white hake

111 (Urophycis tenuis), American plaice (Hippoglossoides platessoides) and thorny skate

112 (Amblyraja radiata) in the southern Gulf of St. Lawrence marine ecosystem in Canada.

113 This study system is exceptionally well studied and data-rich, thus providing long time

114 series of the S-R dynamics of the four study species. Historically, Atlantic cod, American

115 plaice and white hake supported the largest fisheries for groundfish in the southern Gulf of

116 St. Lawrence, and thorny skate was the most abundant and widely distributed skate in this

117 ecosystem. Here, we investigate whether the S-R relationships of these species experience

118 non-stationarities by detecting shifts in the parameters of the S-R relationships, infer

119 timings of the parameter shifts (change points), and quantify the typical lengths and

120 magnitudes of the resulting 'recruitment regimes' as well as the associated changes in the

121 S-R model parameters. In addition to exploring shifts in the S-R model parameters, we

122 account for the uncertainties associated with the change points and regime specific

123 parameters, and propagate the uncertainties into predictions using the posterior predictive

124 distribution. 


\section{2. Material and methods}

\section{2.1. Implementation of shifts in the parameters of the stock-recruitment models}

129 Our model consists of a model for the length of the current regime and a model for the data

130 generating process. The regime length is modelled as a Markov process such that, during

131 the transition from a time step to the next, the regime length can only grow by one (the

132 current regime continues) or go to zero (a new regime is starting). The data generating

133 process is the S-R model. We explored the Ricker S-R model (Ricker 1954) and the

134 Beverton-Holt S-R model (Beverton and Holt 1957). In our analysis, we are interested in

135 sequentially inferring the posterior and smoothed probabilities of the length of the current

136 regime and the posterior probability distributions of the model parameters.

138 The Bayesian online change point detection algorithm (BOCPD; Adams and McKay 2007,

139 Perälä and Kuparinen 2015) detects sudden changes in the parameters of a data generating

140 process. The algorithm processes the data sequentially one time step at a time ('online'),

141 and at each time step a new sequential estimation run is started which ignores the data

142 from previous time steps. Assuming that there is a change point at some time step, one of

143 the runs will start at the correct time step and the idea is that it will obtain high posterior

144 probability because of its better predictive performance. The runs are identified in the

145 algorithm by the number of data points that have been used to update the run-specific

146 posterior distribution of the parameters. The auxiliary variable used to distinguish the runs

147 is called 'run length' and it is denoted by $r_{t} \in\{0, \ldots, t\}$. The algorithm infers the posterior

148 probability distribution of the parameters for all possible regime starting times (or

149 equivalently for all possible run lengths), and the run length posterior probabilities. This

150 means that at time step $t$ there are $t$ run-specific posterior probability distributions for 
151 the parameters together with the posterior probabilities of the $t$ run lengths. The full

152

154

posterior probability distribution of the parameters is a mixture distribution consisting of the run-specific posterior distributions and the mixture weights, i.e. the run length posterior probabilities. Similarly, the full posterior predictive distribution is a mixture of run-specific posterior predictive distributions where the weights are the run length posterior probabilities. In the following we will present the integral quantities constituting the algorithm.

\section{Run length posterior}

We assume that the data generating process depends on covariates (i.e. the spawning stock biomass) and write them explicitly as conditioning factors in the probability density functions. The conditional distrbution of the run length $r_{t}$ at time $t$ given the response variables $y_{1: t}=\left(y_{1}, \ldots, y_{t}\right)$ and covariates $S_{1: t}=\left(S_{1}, \ldots, S_{t}\right)$, i.e. the run length posterior distribution, can be formulated recursively (see supplementary material for derivation)

$$
p\left(r_{t} \mid y_{1: t}, S_{1: t}\right)=\sum_{r_{t-1}=0}^{t-1} \frac{p\left(r_{t} \mid r_{t-1}\right) p\left(y_{t} \mid y_{t-1}^{\left(r_{t-1}\right)}, S_{t-1}^{\left(r_{t-1}\right)}, S_{t}\right) p\left(r_{t-1} \mid y_{1: t-1}, S_{1: t-1}\right)}{p\left(y_{t} \mid y_{1: t-1}, S_{1: t-1}, S_{t}\right)}
$$

where $p\left(r_{t} \mid r_{t-1}\right)$ is the change point prior distribution that contains our prior belief of the probability of a shift. The underlying predictive model (UPM) $p\left(y_{t} \mid y_{t-1}^{\left(r_{t-1}\right)}, S_{t-1}^{\left(r_{t-1}\right)}, S_{t}\right)$ is the predictive density conditional on run length $r_{t-1}$, i.e. the predictive density using the latest $r_{t-1}$ observed response variables $y_{t-1}^{\left(r_{t-1}\right)}$ and the corresponding covariates $S_{t-1}^{\left(r_{t-1}\right)}$. For better readability, the following notation is adopted here and used throughout this paper: $y_{s}^{(r)}=y_{(s-r+1): s}$ and $S_{s}^{(r)}=S_{(s-r+1): s}$. The UPM is defined by the data-generating model, and it weights the probabilities of different run lengths by how well 
172 the predictive density associated with the given run predicts the new response variable $y_{t}$

173 given the new covariate value $S_{t}$. If the new response variable is 'far away' from any of

174 the predictive densities of the previous runs, the prior predictive density

$175 p\left(y_{t} \mid y_{t-1}^{(0)}, S_{t-1}^{(0)}, S_{t}\right)$ will dominate, which serves as an indicator for a start of a new

176 regime. The last term $p\left(r_{t-1} \mid y_{1: t-1}, S_{1: t-1}\right)$ is the run length posterior of the previous

177 time step $t-1$. The term in the denominator $p\left(y_{t} \mid y_{1: t-1}, S_{1: t-1}, S_{t}\right)$ is the normalization

178 factor. The summation is carried out for all possible run lengths from 0 (new regime) to

$179 t-1$ (the longest possible regime so far).

180

\section{Change point prior}

182 We define the change point prior probability or the prior probability of a shift using a

183 constant model

$$
p\left(r_{t} \mid r_{t-1}\right)=\left\{\begin{aligned}
\frac{1}{\lambda}, & \text { if } r_{t}=0 \\
1-\frac{1}{\lambda}, & \text { if } r_{t}=r_{t-1}+1 \\
0, & \text { otherwise }
\end{aligned}\right.
$$

184 where the probability of a change point is thus $\frac{1}{\lambda}$. The change point prior has non-zero

185 probability only for two cases: the run length grows by one, i.e., the previous regime

186 continues, or the run length goes to zero, i.e., a new regime starts. The change point prior

187 contains our a priori belief about the frequency of the regime shifts. We set $\lambda=10$, which

188 means that a priori we expect the shifts to happen on average every 10 years. We examine

189 the sensitivity of the results to the change point prior probability in the supplementary

190 material.

191 
192 Underlying predictive model

193 In this study, we detect change points in the parameters of the Ricker and Beverton-Holt

194 S-R models, which relate the number of recruits $\left(R_{t}\right)$ in a given year $t$ to the spawning

195 stock biomass $\left(S_{t}\right)$ that produced the recruits (Ricker 1954, Beverton and Holt 1957). We

196 model the logarithm of the ratio of the number of recruits and the spawning stock biomass

$197 y_{t}=\log \left(\frac{R_{t}}{S_{t}}\right)$ as a normally distributed random variable

$$
y_{t} \sim N\left(f\left(S_{t}\right), \delta_{t}\right)
$$

198 where the mean is given by the function $f\left(S_{t}\right)$, which depends on the S-R model choice,

199 and the standard deviation is the unknown parameter $\delta_{t}=\sigma / \sqrt{c_{t}}$, where $c_{t}$ is a known

200 constant proportional to the sampling intensity in year $t$. For example, in our application

201 to the southern Gulf of St. Lawrence (see section 2.3), the R and S data are based on catch

202 rates in an annual bottom-trawl survey. Sampling intensity in this survey since 1985 was

203 approximately 2.5 times the sampling intensity in earlier years; accordingly, we set $c_{t}=1$

204 when $t<1985$ and $c_{t}=2.5$ when $t \geq 1985$. Thus, $\sigma$ corresponds to the standard

205 deviation of the error before 1985 (when there was a change in vessel and survey

206 protocols).

207

208 The likelihood function of the parameters $(\alpha, \beta, \sigma)$ given a single response variable and

209 covariate pair $\left(y_{t}, S_{t}\right)$ can be thus written as

$$
L\left(\alpha, \beta, \sigma \mid y_{t}, S_{t}\right)=p\left(y_{t} \mid S_{t}, \alpha, \beta, \sigma\right)=\frac{1}{\sqrt{2 \pi} \delta_{t}} e^{-\frac{1}{2 \delta_{t}^{2}}\left(y_{t}-f\left(S_{t}\right)\right)^{2}} .
$$

210

211 Ricker stock-recruitment model 
212 The Ricker S-R model is usually presented as

$$
R_{t}=\alpha^{\mathrm{R}} S_{t} e^{-\beta^{\mathrm{R}}} S_{t}
$$

213 where the parameter $\alpha^{\mathrm{R}}$ is the slope of the function at the origin and it corresponds to the

214 maximum recruit production per unit of $S_{t}$ in the absence of density-dependent factors.

215 The parameter $\beta^{\mathrm{R}}$ describes the magnitude of the density dependency. The number of

216 recruits first grows as a function of the spawning stock biomass until the maximum

217 number of recruits or 'carrying capacity for the recruits' is reached at $S_{t}=\frac{1}{\beta^{\mathrm{R}}}$ after which

218 the number of recruits starts declining. The carrying capacity for the number of recruits is

219 then $k=\frac{\alpha^{\mathrm{R}}}{e \beta^{\mathrm{R}}}$. For the Ricker S-R model the mean in (5) is

$$
f\left(S_{t}\right)=\log \left(\alpha^{\mathrm{R}}\right)-\beta^{\mathrm{R}} S_{t}
$$

220

221

Beverton-Holt stock-recruitment model

222 The Beverton-Holt S-R model can be formulated as

$$
R_{t}=\frac{\alpha^{\mathrm{BH}} S_{t}}{1+\beta^{\mathrm{BH}} S_{t}}
$$

223 where $\alpha^{\mathrm{BH}}$ is the slope of the function at origin and $\beta^{\mathrm{BH}}$ controls the

224 density-dependence. Recruitment in the Beverton-Holt model approaches an asymptotic

225 value of $R_{\infty}=\frac{\alpha^{\mathrm{BH}}}{\beta^{\mathrm{BH}}}$ as the spawning stock biomass grows. When $S_{t}=\frac{1}{\beta^{\mathrm{BH}}}$ the

226 recruitment is $\frac{1}{2} R_{\infty}$. For the Beverton-Holt S-R model the mean in (5) is

$$
f\left(S_{t}\right)=\log \left(\alpha^{\mathrm{BH}}\right)-\log \left(1+\beta^{\mathrm{BH}} S_{t}\right)
$$

227

228 In the framework of BOCPD and the S-R models presented above, we want to make

229 inference about the S-R model parameters $\alpha$ and $\beta$, and the standard deviation of the 
230 error distribution $\sigma$ using the latest $r_{t}$ observed response variables $y_{t}^{\left(r_{t}\right)}$ and covariates

$231 S_{t}^{\left(r_{t}\right)}$. Bayesian inference is based on calculating the joint distribution of the S-R model

232 parameters and the standard deviation of the error distribution conditioned on the observed

233 response variables and covariates, $p\left(\alpha, \beta, \sigma \mid y_{t}^{\left(r_{t}\right)}, S_{t}^{\left(r_{t}\right)}\right)$, i.e. the posterior distribution of

234 the parameters given run length $r_{t}$ at time step $t$. The posterior distribution for each run

235 length $r_{t} \in\{1, \ldots, t\}$ is calculated recursively by sequential application of the Bayes' rule.

236 The recursion starts from a fixed prior distribution $p\left(\alpha, \beta, \sigma \mid y_{t-1}^{(0)}, S_{t-1}^{(0)}\right)=p(\alpha, \beta, \sigma)=$

$237 p(\alpha) p(\beta) p(\sigma)$, where the parameters are assumed a priori independent. The prior

238 distributions are provided as an input for the algorithm. The calculation of the posterior

239 distributions of the parameters is analytically intractable, and thus requires approximate

240 numerical methods. Due to the sequential nature of the BOCPD algorithm, we use

241 sequential Monte Carlo (SMC) or particle filter methods for infering the posterior

242 parameter distributions. However, particle filters suffer from particle degeneracy issues

243 when estimating static parameters. Thus, we use artificial evolution of the parameters to

244 overcome this issue (Liu and West, 2001). Furthermore, to calculate the run length

245 posterior, the posterior predictive density (i.e. the probability density of a new response

246 variable $y_{t+1}$ conditional on a new covariate $S_{t+1}$ and the $r_{t}$ latest response variables

247 and covariates) $p\left(y_{t+1} \mid y_{t}^{\left(r_{t}\right)}, S_{t}^{\left(r_{t}\right)}, S_{t+1}\right)$ needs to be evaluated. The evaluation of the

248 posterior predictive density involves 3-dimensional integrals, which can be solved

249 approximately within the SMC framework.

250

251 Sequential Monte Carlo algorithm 
252 The run-specific posterior distributions are analytically intractable and need to be 253 calculated using approximate numerical methods. We inferred the posterior distributions 254 using a sequential Monte Carlo algorithm. The number of particles was set to $N=$ 255 100000. To avoid particle degeneracy, the particle locations were resampled at each time 256 step using the idea of artificial evolution of parameters (Liu and West, 2001), so that the 257 number of effective particles after each time step was at least 50000. The artificial 258 evolution of the parameters was accomplished by moving the particles around in the 259 parameter space by drawing new particle locations from multivariate normal kernels 260 whose means are shifted from the particle locations towards the sample mean of the 261 particle locations. The magnitude of the shift is governed by a smoothing parameter $2620<h<1$. The smoothing parameter was chosen arbitrarily, and since the results seemed 263 reasonable, its role was not examined further nor was it tuned in any way. The resampling 264 was quite efficient, and it was usually needed to be done only once per time step. The 265 SMC algorithm works as follows. We only present the update for a single time step. The 266 update is carried out similarly at each time step (excluding step 0 , which is carried out only 267 before a sequential estimation run starts).

2680 ) Set the smoothing parameter $h=0.1$ and calculate $a=\sqrt{1-h^{2}}$. Draw $N$ 269 parameter vectors (particle locations) $\theta_{0}^{(j)}=\left(\alpha^{(j)}, \beta^{(j)}, \sigma^{(j)}\right), j=1, \ldots, N$ from

270 the prior and set the corresponding particle weights to $w_{0}^{(j)}=\frac{1}{N}$.

271 Repeat 1-10 until the number of effective particles $\left(N_{t}^{\text {eff }}\right)$ is at least 5000: 
1) Take the natural logarithm of the parameter vectors from the previous time step $\vartheta_{t-1}{ }^{(j)}=\log \left(\theta_{t-1}^{(j)}\right)$ to avoid negative values for the non-negative parameters later on.

2) Calculate the sample mean and covariance matrix from the log-transformed particle locations $\mu_{t}=\mathrm{E}\left(\vartheta_{t-1}\right)$ and $\Sigma_{\mathrm{t}}=\operatorname{Cov}\left(\vartheta_{t-1}\right)$.

3) Calculate the means of the kernel locations in log-space as

$$
m_{t}^{(j)}=a \vartheta_{t-1}^{(j)}+(1-a) \mu_{t}, j=1, \ldots, N
$$

4) Transform back to normal space: $\xi_{t}^{(j)}=\exp \left(m_{t}^{(j)}\right)$.

5) Calculate weights proportional to the product of the likelihood function evaluated at the kernel means and the weights at the previous time step

$$
\omega_{t}^{(j)} \propto w_{t-1}^{(j)} L\left(\xi_{t}^{(j)} \mid y_{t}, S_{t}\right), \quad j=1, \ldots, N
$$

281 6) Sample $N$ indices from a multinomial distribution according to the weights

$$
I_{t}^{(i)} \sim \text { Multinomial }\left(\{1, \ldots, N\},\left\{\omega_{t}^{(1)}, \ldots, \omega_{t}^{(N)}\right\}\right), \quad i=1, \ldots, N
$$

7) Draw new particle locations in the log-space from a multivariate normal distribution (and check that they belong to the support of the prior distributions, otherwise draw again)

$$
\vartheta_{t}^{(i)} \sim \operatorname{MVN}\left(m_{t}^{\left(I_{t}^{(i)}\right)}, h^{2} \Sigma_{\mathrm{t}}\right), i=1, \ldots, N
$$

8) Transform back to original space to get the new particle locations $\theta_{t}^{(i)}=$ $\exp \left(\vartheta_{t}^{(i)}\right)$

9) Calculate the new particle weights proportional to the ratio of the likelihoods evaluated at the kernel means and at the new particle locations 


$$
w_{t}^{(i)} \propto \frac{L\left(\xi_{t}^{\left(I^{(i)}\right)} \mid y_{t}, S_{t}\right)}{L\left(\theta_{t}^{(i)} \mid y_{t}, S_{t}\right)}
$$

289

10) Calculate the number of effective particles

$$
N_{t}^{\mathrm{eff}}=\frac{1}{\sum_{i=1}^{N}\left(w_{t}^{(i)}\right)^{2}}
$$

290

$291 \quad$ Algorithm outputs

292 The output of the algorithm consists of the posterior run length probabilities and the

293 posterior distributions of the parameters for each run. The full posterior distribution of the

294 parameters is a weighted sum of the run-specific posterior distributions of the parameters

295 where the weights are the posterior probabilities of the run lengths $r_{t}$ (3) (see

296 supplementary material for derivation). Similarly, the posterior predictive density of a new

297 response variable $y_{t+1}$ given a new covariate value $S_{t+1}$ is a weighted sum of the regime

298 specific posterior predictive densities. The posterior predictive density is an integral

299 quantity when predicting the recruitment in the future.

300

301 Identifying recruitment regimes

302 The algorithm provides the run length posterior probabilities which can be used as an early

303 indicator of a change point. If the posterior probability that the run length is one is high, it

304 means that the older runs do not explain the new data point well, which suggests that a

305 shift in the parameters has happened. However, high posterior probability can also result if

306 the most recent data point is an outlier caused for example by a gross error in the survey 
307 process. In retrospect, it is also possible to calculate the smoothed run length probabilities

$308 p\left(r_{\tau} \mid y_{1: t}, S_{1: t}\right)$ for any $\tau<t$ recursively using the following equations (Särkkä 2013):

$$
\begin{gathered}
p\left(r_{\tau+1} \mid y_{1: \tau}, S_{1: \tau}\right)=\sum_{r_{\tau}=0}^{\tau} p\left(r_{\tau+1} \mid r_{\tau}\right) p\left(r_{\tau} \mid y_{1: \tau}, S_{1: \tau}\right) \\
p\left(r_{\tau} \mid y_{1: t}, S_{1: t}\right)=p\left(r_{\tau} \mid y_{1: \tau}, S_{1: \tau}\right) \sum_{r_{\tau+1}=0}^{\tau+1} \frac{p\left(r_{\tau+1} \mid r_{\tau}\right) p\left(r_{\tau+1} \mid y_{1: t}, S_{1: t}\right)}{p\left(r_{\tau+1} \mid y_{1: \tau}, S_{1: \tau}\right)} .
\end{gathered}
$$

309 The smoothed run length probabilities are not affected by single outliers. After observing

310 the entire data $\left(y_{1: T}, S_{1: T}\right)$ we can update our belief about the change points by examining

311 the smoothed run length probabilities. The algorithm is probabilistic, and we could choose

312 any number of criteria to divide the data into regimes. We use the smoothed run length

313 probabilities to find the most likely segmentation of the data, which is described next.

315 The most likely segmentation of data

316 We divide the data into regimes by calculating the most likely segmentation of data based

317 on the smoothed run length probabilities. The segmentation is an ordered set of run

318 lengths. An example for a dataset of length 10 could be $(1,2,3,4,5,6,1,2,3,4)$. Here, the

319 first 6 data points belong to the first regime, and the last 4 data points belong to the second

320 regime.We go through all possible such segmentations so that the maximum number of

321 regimes is 5 . We look only at the cases where the minimum length of a regime is 3 years,

322 except for the first and the last regime, since the data cannot determine when the first

323 regime started or the last regime ended. The most likely segmentation is found by

324 maximizing the product of the smoothed run length probabilities of a segmentation. In the

325 above example the product would be $\prod_{i=1}^{6} p\left(r_{i}=i\right) \prod_{i=1}^{4} p\left(r_{6+i}=i\right)$. 


\section{Choosing prior distributions}

328 For the algorithm to perform optimally, there are some considerations related to the 329 parameter prior distributions. Firstly, since the posterior probability of the start of a new

330 run depends on the value of the prior predictive density evaluated at the new observation,

331 the prior should be a proper probability distribution. Secondly, the value of the prior

332 predictive density should be high enough for all the observations compared to the posterior

333 predictive densities in order for the algorithm to yield enough probability for the new runs.

334 Thirdly, we want to assume that all observations are about as likely based on the prior

335 predictive density, since a priori we do not have any reason to assume otherwise. We also

336 want the observations to be about equally likely for both S-R models. Finally, since the

337 S-R models can be parameterized in several different ways, we do not want the inference

338 to depend on the particular parameterization. To accomplish the above-mentioned goals,

339 we build our prior distributions using the Jeffreys' method (Jeffreys, 1964). The Jeffreys'

340 priors depend on the likelihood function for the parameters, and are thus different for each

341 model. They are invariant to the choice of model parameterization, and produce flat prior

342 predictive distributions. Such priors have been formulated for the Ricker and

343 Beverton-Holt S-R models (Millar, 2002). However, these priors are improper, and thus do

344 not suit our needs. Our solution to this problem is to constrain the priors to a predefined

345 range of the parameters. The priors we use for the Ricker model are

$$
\begin{aligned}
& p\left(\alpha^{R}\right) \propto \frac{1}{\alpha^{\mathrm{R}}}, \quad \alpha^{\mathrm{R}} \in\left[\alpha_{\mathrm{up}}^{\mathrm{R}}, \alpha_{\mathrm{low}}^{\mathrm{R}}\right], \\
& p\left(\beta^{\mathrm{R}}\right) \propto 1, \quad \beta^{\mathrm{R}} \in\left[\beta_{\mathrm{low}}^{\mathrm{R}}, \beta_{\mathrm{up}}^{\mathrm{R}}\right], \\
& p\left(\sigma^{\mathrm{R}}\right) \propto \frac{1}{\sigma^{\mathrm{R}}}, \quad \sigma^{\mathrm{R}} \in\left[\sigma_{\mathrm{low}}^{\mathrm{R}}, \sigma_{\mathrm{up}}^{\mathrm{R}}\right] .
\end{aligned}
$$


346 For the Beverton-Holt model the priors are

$$
\begin{gathered}
p\left(\alpha^{\mathrm{BH}}\right) \propto \frac{1}{\alpha^{\mathrm{BH}}}, \quad \alpha^{\mathrm{BH}} \in\left[\alpha_{\mathrm{low}}^{\mathrm{BH}}, \alpha_{\mathrm{up}}^{\mathrm{BH}}\right], \\
p\left(\beta^{\mathrm{BH}}\right) \propto V\left(g\left(\beta^{\mathrm{BH}}\right)\right)^{\frac{1}{2}}, \quad \beta^{\mathrm{BH}} \in\left[\beta_{\text {low }}^{\mathrm{BH}}, \beta_{\mathrm{up}}^{\mathrm{BH}}\right], \\
p\left(\sigma^{\mathrm{BH}}\right) \propto \frac{1}{\sigma^{\mathrm{BH}}}, \quad \sigma^{\mathrm{BH}} \in\left[\sigma_{\mathrm{low}}^{\mathrm{BH}}, \sigma_{\mathrm{up}}^{\mathrm{BH}}\right],
\end{gathered}
$$

347 where $V\left(g\left(\beta^{\mathrm{BH}}\right)\right)=\frac{1}{T-1} \sum_{t}\left(g_{t}\left(\beta^{\mathrm{BH}}\right)-\frac{1}{T} \sum_{t} g_{t}\left(\beta^{\mathrm{BH}}\right)\right)^{2}$ is the sample variance operator,

348 and $g_{t}\left(\beta^{\mathrm{BH}}\right)=\frac{S_{t}}{1+\beta^{\mathrm{BH}} S_{t}}, t=1, \ldots, T$. The upper and lower limits were chosen so that the 349 ranges would contain all the plausible values for the parameters. The $\alpha$ parameter, which 350 represents the slope of the S-R curve at the origin was restricted so that the minimum and

351 the maximum of $R / S$ in the data fell into the interval. For the $\beta$ parameter, we chose the

352 limits so that the resulting carrying capacity $k$ in the Ricker model and the asymptotic

353 value $R_{\infty}$ in the Beverton-Holt model would fall into an interval where we believe these

354 quantities could be. In addition, the ranges were still widened so that on average each

355 observation for every species and for both S-R models was about equally likely based on

356 the prior predictive density. The upper and lower limits for the parameter priors are listed

357 in Table 1. Since the choice for the support of the parameters is subjective, we examine the

358 robustness of the results to different choices for the supports. The description of the

359 sensitivity analyses is presented in the supplementary material.

360

361 Missing data 
362 In case there is missing data for some years, we predict the run length posterior

363 distribution for those years using the one-step prediction equation (11). The run-specific

364 posterior distributions of parameters are assumed not to change during the years of missing

365 data. However, a new estimation run is also started during these years, to account for the

366 possibility of a regime shift during the missing year.

367

368 2.2. Simulation setup

369 To validate our method we generated simulated data using the Beverton-Holt and Ricker

370 stock-recruit models. We set the length of the simulation time series $T=40$, which

371 roughly corresponds to the length of our empirical time series. We drew randomly the

372 number of regimes $n^{r}$ from the set $\{2,3,4\}$. Next, we divided the time interval $\{1, \ldots, T\}$

373 into $n^{r}$ regimes with regime lengths $l_{i}^{r}, i=1, \ldots, n^{r}$ randomly chosen from $\{5,6, \ldots, 20\}$

374 so that the sum of the regime lengths $\sum_{i=1}^{n^{r}} l_{i}^{r}=T$. Next, we generated the regime specific

375 parameters such that every other change in $\alpha$ was positive and every other was negative.

376 The magnitudes of the relative changes were drawn from $U(0.5,4)$ for the positive

377 changes and from $U(-0.8,-0.2)$ for the negative changes with the constraint that the

378 new $\alpha \in[0.1,5]$. For simplicity for the first regime $\alpha^{\mathrm{R}}=\alpha^{\mathrm{BH}}=1$ in each simulated

379 data set and the density dependence parameters $\beta^{\mathrm{R}}=7.4 \cdot 10^{-9}$ and $\beta^{\mathrm{RH}}=14 \cdot 10^{-9}$

380 were constant during the whole simulation data set. The SSB values were drawn from

$381 U\left(0,3 \cdot 10^{8}\right)$, and the corresponding $\log \left(\frac{R}{S}\right)$ values were drawn from $N(f(S), \sigma)$. We

382 tested four different noise levels $\sigma \in\{0.3,0.4,0.5,0.6\}$. For each level of noise, we

383 generated 100 data sets. Overall, we had thus 400 simulated time series of $(S, R)$ pairs

384 for both S-R models. 
385 We fitted the S-R models to the simulated data using BOCPD, and for comparison using

386 models with time-invariant parameters. The models with time-invariant parameters were

387 fitted using Markov Chain Monte Carlo methods. For each simulated data set, we

388 calculated the proportion of time steps when the true parameter values were inside the

389 estimated $68 \%$ and $95 \%$ posterior central probability intervals (CPI) and the root mean

390 squared error (RMSE) of the posterior median estimate. We calculated similar scores for

391 the prediction ability of the models at each time step by comparing the true recruitment to

392 the posterior predictive distribution of the previous year given the true spawning stock

393 biomass. We also calculated how many of the change points were found correctly, how

394 many times they were found within two time steps of their actual occurrence, and how

395 many times the change point was completely missed by our method. We also calculated

396 the number of false alarms, i.e., number of change points reported by our method when

397 there actually was none.

399 2.3. Empirical S-R data

400 To explore non-stationarities in S-R dynamics of Atlantic cod, thorny skate, American

401 plaice, and white hake (hereafter denoted as cod, skate, plaice, and hake, respectively), we

402 use indices of recruit abundance and spawning stock biomass of these fishes in the

403 southern Gulf of St. Lawrence (sGSL). Data on population abundance and age and size

404 composition were obtained for these species by a bottom-trawl survey conducted by the

405 Canadian Department of Fisheries and Oceans (DFO) each September since 1971. This

406 survey follows a stratified-random design, with stratification based on depth and 
407 geographic area (Fig. S1). Sampling stations are allocated to strata in rough proportion to 408 stratum area. The standard fishing procedure was a 30 -min tow at 3.5 knots in all years. 409

410 A change in survey gear occurred in 1985 and the survey vessel changed in 1985, 1992 411 and 2004. Comparative fishing experiments were conducted to calibrate the old and new 412 vessels and gear. When a change in fishing efficiency was detected for a particular species, 413 catches by the earlier vessel/gear were adjusted to be equivalent to those by the new 414 vessel/gear (Benoît and Swain 2003a; Benoît 2006). An uncalibrated vessel conducted the 415 survey in 2003, and some strata were not sampled. Thus, we omitted the data for this year. 416 Consequently, two years have missing data for each stock. One is 2003 and the other one 417 is the year in which the recruits are assumed to be produced by the spawning stock of 418 2003. For white hake, the estimated change in fishing efficiency in 1985 was not 419 significant, though statistical power was low due to its restricted distribution. Recent 420 population modelling indicated a change in fishing efficiency for this species similar to the 421 non-significant effect estimated by comparative fishing (Swain et al. 2015). Catch rates of 422 this species prior to 1985 were adjusted to account for this change in fishing efficiency.

424 Fishing during the survey was conducted during daylight hours only (07:00 - 19:00) prior 425 to 1985 and 24-h per day since then. Diel differences in catchability were assessed based 426 on paired day and night survey tows conducted at the same locations and on analysis of the $427 \quad 1985-2001$ survey data controlling statistically for annual and spatial variation in catch 428 rates. Estimated species-specific diel effects were similar between approaches, time 429 periods and vessels, and were comparable to results from studies in other areas (Benoit and 430 Swain 2003b). When diel differences in catchability occurred, night catches were adjusted 
431 to be equivalent to day catches in order to maintain a consistent time series (Benoît and

432 Swain 2003a; Benoît 2006). The sampling intensity of the surveys also changed in 1985.

433 Before $1985,64-108$ tows were conducted annually (mean = 70.6) and since 1985 the

434 number of tows has increased to $132-244($ mean $=180.8)$. The survey provides data on the

435 mean weight of individuals in each age class $w_{a, t}$, the proportion of mature individuals in

436 each age class $m_{a, t}$, and the mean number of individuals per tow in each age class $n_{a, t}$.

438 The estimate of the number of recruits in year $t\left(R_{t}\right)$ is calculated as the mean number of

439 individuals of age $a=a^{R}$ per tow, multiplied by the number of trawlable units in the

440 survey area $(U=1729346)$, where $a^{R}$ is the assumed age of recruitment. The ages of

441 recruitment used for the study species are given in Table 1.

$$
R_{t}=N_{a^{R}}=n_{a^{R}} U
$$

442 where $n_{a^{R}}$ is the stratified mean number of recruits per standard tow in year $t$ and $N_{a^{R}}$

443 is the survey estimate of recruit abundance ("trawlable" abundance) in year $t$. The

444 estimate of the spawning stock biomass, which produced the recruits, is calculated as

$$
S_{t}=\sum_{a=0}^{a_{\max }} w_{a, t-a^{R}} m_{a, t-a^{R}} N_{a, t-a^{R},}
$$

445 where $w_{a, t}$ is the estimate of the mean weight of individuals in age class $a$ in the

446 beginning of year $t, m_{a, t}$ is the estimated proportion of mature individuals in age class $a$

447 in year $\mathrm{t}$, and $N_{a, t}$ is the estimate of the number of individuals in age class $a$ in year $t$.

448 The mean weight at age in the beginning of the year $t$ is calculated as the geometric mean

449 of the September mean weight at age of the same cohort in years $t-1$ and $t$. Survey 
450 indices of recruit abundance and spawner biomass are given in Table S1 along with

451 measures of their uncertainty.

452

\section{3. Results}

454 The methods ability to detect the change points in the simulated data depended on the 455 magnitude of the change in $\alpha$ and the level of noise. The smaller the change in $\alpha$ and the 456 higher the noise level the more difficult it was to detect the change points. (Figs. 1, S5).

457 The number of false alarms was positively correlated with the noise level. In the

458 Beverton-Holt model simulations, the number of false alarms were 10, 35, 59 and 71,

459 when the noise levels were 0.3, 0.4, 0.5 and 0.6, respectively. For the Ricker model

460 simulations, the number of false alarms were 15, 29, 44 and 50. To put these numbers in

461 perspective, the total number of change points in the Beverton-Holt and Ricker simulations 462 were 774 and 821 , respectively.

463 The estimated posterior distributions for the parameters were much closer to the true 464 parameter values for the BOCPD model than for the model with time-invariant parameters 465 based on the frequency with which the true parameter value was inside the $68 \%$ and $95 \%$ 466 posterior CPIs (Fig. 2 \& S6). The performance of the methods got worse as the level of 467 noise got higher.

468 In addition, the medians of the parameter posterior marginal distributions produced by 469 BOCPD were consistently closer to the true parameter values than those produced by the 470 time-invariant model (Table 2.). 
471 The BOCPD model predictions were also better than the predictions of the constant

472 parameter model, both based on the frequency with which the next year's recruitment was

473 inside the CPIs of the posterior predictive distribution, and the RMSE of the median

474 estimates (Fig. 2 \& S6, Table 2).

475 In our empirical data, we found shifts in the parameters of both of the S-R models in all

476 four study species. The number of detected regimes based on the most likely segmentation

477 of the data ranged from two to four, and the lengths of the regimes for which both the start

478 and end years were identified ranged from 4 to 21 years (Fig. 3). The shifts radically

479 changed the marginal posterior distributions of the parameters (Figs. 4, S2, and S3). The

480 shifts were mainly driven by changes in $\alpha$, while $\beta$ remained relatively constant.

481 Because of this, the changes in carrying capacity, $k$, and the asymptotic maximum, $R_{\infty}$

482 reflect the changes in the maximum recruitment per unit of SSB, $\alpha$ (Fig. 5). The

483 parameter estimates for $\alpha^{\mathrm{R}}$ and $\alpha^{\mathrm{BH}}$ are almost identical, which shows that the method

484 is not very sensitive to the choice of the S-R model in the studied stocks. This is also seen

485 in the timings of the change points based on the most likely segmentation of the data (Fig.

486 3). Uncertainty related to the regime-specific parameters in the beginning of a new regime

487 was also reflected as an increase in the standard deviation parameter, $\delta_{t}$, immediately

488 after the shifts (Fig. S3). The changes in the parameters also resulted in clearly different

489 medians of the posterior predictive distributions of $R_{t}$ at the end of the detected regimes

490 (Fig. 6). The medians of the posterior predictive distributions of $\log \left(\frac{R_{t}}{S_{t}}\right)$ are shown in

$491 \quad$ Figure S4.

492 
493 For cod, we found shifts in 1976, 1991 and 2013 using the Ricker model (Fig. 3a). The

494 posterior median estimates of $\alpha^{\mathrm{R}}$ at the end of the four resulting regimes were 0.42 ,

$4951.31,0.31$, and 2.89 , respectively. In terms of relative change in $\alpha^{\mathrm{R}}$ the magnitude of

496 the shifts were $212 \%,-76.6 \%$, and $839 \%$. Using the Beverton-Holt model, the shifts

497 occurred in 1991 and 2013 (Fig. 3b), and the posterior median estimates of $\alpha^{\text {BH }}$ at the

498 end of the three resulting regimes were $1.30,0.35$, and 3.01 , respectively. In terms of

499 relative change in $\alpha^{\mathrm{BH}}$ the magnitude of the shifts were $-72.7 \%$, and $749 \%$.

500

501 The shifts found for hake occurred in 1979 and 1997 for both S-R models (Fig. 3c-d). The

502 shifts were driven by changes in $\alpha$ (Fig. 4), although the density dependence parameter $\beta$

503 also tended to be higher for the third regime than for the earlier regimes based on the

504 Ricker model (Fig. S2). The posterior median estimates of $\alpha^{\mathrm{R}}$ at the end of the three

505 resulting regimes were $2.37,0.71$ and 1.92 , respectively. This resulted in relative

506 change in $\alpha^{\mathrm{R}}$ of $-69.8 \%$ and $169 \%$. The corresponding posterior median estimates of

$507 \alpha^{\mathrm{BH}}$ were $2.95,0.84$, and 2.05 , which amount to relative changes of $-71.4 \%$ and $508143 \%$.

509

510 Plaice experienced a shift in 1982 when analyzed using the Ricker model (Fig. 3e). The

511 posterior median estimates of $\alpha^{\mathrm{R}}$ at the end of the two resulting regimes were 4.73 and

5122.36 , respectively. In terms of relative change, the magnitude of this shift was $-50 \%$.

513 Using the Beverton-Holt model, we found shifts in 1982 and 1986 (Fig. 3f). The posterior

514 median estimates of $\alpha^{\mathrm{BH}}$ at the end of the three resulting regimes were $4.46,1.27$, and 
515 2.70. The magnitude of the shifts in terms of relative change were thus $-71.4 \%$ and $516110 \%$.

518 The data point of 1984 in the skate time series seemed to be an outlier, and we removed it

519 from the data set before running the analysis. The shifts for skate as identified by our 520 change point detection scheme occurred in 1988, 1999 and 2006 using both S-R models

521 (Fig. 3g-h). The posterior median estimates of $\alpha^{\mathrm{R}}$ at the end of the four regimes were $0.27,0.72,0.47$, and 1.98 , respectively. Translated into relative changes the magnitude

523 of the shifts were $169 \%,-34.8 \%$, and $324 \%$. The posterior median estimates of $\alpha^{\mathrm{BH}}$

524 were $0.26,0.83,0.50$, and 2.13 . The relative changes between regimes were thus

$525222 \%,-40 \%$, and $329 \%$.

526

527 The sensitivity analyses showed that increasing the change point prior probability to 0.2

528 resulted in more change points for each stock. On the other hand, when using smaller

529 change point prior probability some of the change points were not detected (Table S2).

530 However, change points were found for each stock using both S-R models with $\lambda=20$.

531 Most notably, even with $\lambda=10000$ the two change points in 1991 and 2013 were found

532 for cod with both S-R models (Fig. S10). This indicates that there is amble evidence of at

533 least two change points in the data. Moreover, as $\lambda$ increased, the parameter posterior

534 marginal distributions showed less year to year variation (Fig. S11), however the

535 distributions at the end of the regimes did not differ much, which is also seen as almost

536 identical medians of the posterior predictive distributions of $R_{t}$ at the end of the detected

537 regimes (Fig. S12). The results were even more robust to widening the support of the

538 parameters. In some cases, one less change point was found, whereas in some cases one 
539 more change point was found (Table S2). Only in the case of plaice, the change of the

540 support affected the timing of the shifts, when the width of the support of $\alpha^{\mathrm{BH}}$ was

541 doubled. In general, the widening of the supports did not affect the parameter posterior

542 marginal distributions notably (Fig. S14), with the exception that in cases where the data

543 carried little information about the density dependece parameter $\beta$, the uncertainty about

$544 \beta$ was increased as the support of $\beta$ was increased (Fig. S15). The resulting medians of

545 the posterior predictive distributions, however, differed very little (Fig. S16).

546

\section{4. Discussion}

548 All four of the studied stocks showed shifts in their S-R parameters during the years

549 1973-2014 (Fig. 3). In comparison with the constant model, which assumes time-invariant

550 parameters, it is clear that the BOCPD predictive distributions at the end of the regimes fit

551 the data much better than the constant model (Fig. 6). This is because the constant

552 parameter model gives equal weight to all observations, whereas the BOCPD model tends

553 to give more weight to the most recent observations when there is evidence of a recent

554 change point. Furthermore, the constant parameter model consistently produces smaller

555 uncertainty in the parameter estimates (Fig. 4), such that as more data accumulates the

556 uncertainty in the constant parameter model estimates is reduced. This prevents the

557 estimate from changing much when a shift in the parameter value happens, and is most

558 apparent in the posterior marginal density of $\alpha$ parameters at the beginning of new

559 regimes. When a shift occurs, the BOCPD posterior median estimate rapidly shifts to a

560 new level. The posterior median estimate of the constant parameter model also tends to

561 shift toward the new level, but this shift occurs much more slowly and the new level may 
562 not be reached even 20 years after the shift (e.g. the third regime for the Ricker cod

563 model).

564

565 In the light of the findings of the present study, the commonly assumed stationarity in S-R

566 relationships may often not be valid. Our study showed that shifts in S-R dynamics were

567 common among the four fish species examined, and in the Gulf of St. Lawrence, they can

568 change the level of maximum recruit production per unit of SSB by a factor of eight.

569 While the present study was limited to one marine ecosystem, indications of

570 non-stationarities in the parameters of S-R models have been previously documented in the

571 North Sea (Olsen et al. 2011) and the Pacific Ocean (Munch and Kottas 2009). The present

572 study goes deeper by exploring the nature of temporally shifting dynamics and by

573 presenting novel and flexible methodology. In the following, we discuss our findings with

574 respect to changes seen in the cod, hake, skate and plaice stock dynamics in the Gulf of St.

575 Lawrence, in order to explore potential causes and pathways of shifts. Secondly, we

576 compare our modelling approach to previous attempts to account for temporal changes in

577 S-R dynamics and sketch future directions towards accounting for parameter shifts in

578 fisheries stock assessments.

579

580 Regime shifts are often defined as relatively sudden changes between alternate and

581 persistent (commonly 10-30 years) ecosystem states, involving multiple variables and wide

582 spatial scales (de Young et al. 2008, Jiao 2009, Möllmann et al. 2015). These shifts are

583 often attributed to decadal climate-ocean oscillations which move the ecosystem between

584 two alternate stable states (Jiao 2009). In fact, some scientists reserve the term "marine

585 ecosystem regime shift" to cases linked to climatic forcing (Beamish et al. 2004). 
586 However, fishing is now also recognised as an important driver of marine ecosystem

587 regime shifts (Möllman et al. 2008, Jiao 2009). For example, direct and indirect effects of

588 harvesting appear to be important drivers of a "regime shift" in the composition of the

589 marine fish community in the southern Gulf of St. Lawrence, from a community

590 dominated by large-bodied fishes to one dominated by small-bodied fishes (Benoît and

591 Swain 2008).

592

593 This study focusses on "regime shifts" in the recruitment dynamics of single species within

594 an ecosystem. In most cases, the identified regimes are "persistent", lasting for an average

of 16-17 years (omitting regimes ending within 10 years of the start of the time series and

596 those starting within 10 years of the end of the time series). They are also consistent with

597 the timing of fishing-induced changes in this ecosystem. A high productivity regime was

598 identified for cod beginning in the mid-1970s and lasting throughout the 1980s (Fig. 4).

599 This coincides with a collapse in the biomass of pelagic fishes in this ecosystem due to

600 overfishing in the 1960s and early 1970s. These fishes appear to have a negative impact on

601 cod recruitment success through predation on the early-life stages of cod (Swain and

602 Sinclair 2000). Pelagic fish biomass recovered in the 1980s, and cod recruitment rates

603 returned to more normal levels in the 1990s. The recruitment rate of White hake has been

604 exceptionally high since the mid-1990s (Swain et al. 2015). This is interpreted as very high

605 survival of small hake since the mid-1990s. A similar pattern of improved survival of

606 small fish is seen throughout the marine fish community of the southern Gulf during this

607 period, and may reflect a release from predation following the collapse of large demersal

608 fish in this ecosystem (Benoît and Swain 2008). The recent period of exceptionally high 
609 recruitment rates of hake corresponds with the positive shift in $\alpha$ in 1997 (Fig. 4). Based

610 on population modelling results, Morin et al. (2013) suggested that the S-R relationship of

611 plaice has been stationary since 1976 . However, they also noted that the established S-R

612 relationship might not describe the dynamics of the stock adequately in a more productive

613 period prior to 1976 . Our study is in line with these conclusions, providing no evidence for

614 a shift in the R-S relationship of this stock since 1982 or 1986 depending on the S-R model

615 used. Consistent with the suggestions of Morin et al. (2013), our results indicate a shift

616 from a more productive to a less productive regime in 1982 (Fig. 4). It has been reported

617 that the productivity of Thorny skate at early life history stages increased from the 1970s

618 to the 1990s and remained high since then (Swain et al. 2013). Swain et al. (2013) could

619 not distinguish between effects due to increased recruitment rate versus increased survival

620 of post-recruit juveniles. Based on our study (Fig. 4), high productivity at early life stages

621 appears to have been mainly due to high survival of post-recruit juveniles in the 1990s and

622 early 2000s (when our estimated S-R parameters changed little), whereas high recruitment

623 success may have been the main contributing factor since the mid-2000s (when a shift to a

624 higher productivity regime is estimated by our model).

625

626 Regimes are often modelled as alternating between two stable states (e.g. Steele 1996,

627 Munch and Kottas 2009). This is not necessarily the case for "real" systems (Steele 1996),

628 particularly when there is an interplay between fishery and climate impacts (Jiao 2009).

629 Our method places no restriction on the number of distinct regimes. It can be used when

630 the system oscillates between two alternate states but is not restricted to this case. In our

631 hake example, estimated recruitment productivity shifted from a high level in the 1970s to 
632 a low level, and then back to a high level since the mid-1990s. However, the recent state of

633 high recruitment success for hake is not a return to the earlier state; both the ecosystem and 634 the hake population differed greatly between these two periods (Benoît and Swain 2008,

635 Swain et al. 2016).

636

637 The smoothed probabilities of the start of new regimes (Fig. 3) and the parameter posterior 638 marginal densities (Fig. 4) reveal an interesting synchronicity in the timings of the shifts

639 between species. Atlantic cod is the dominant predatory fish in this ecosystem. Cod

640 biomass declined from the mid-1950s to the mid-1970s due to overfishing (Swain and

641 Benoit, 2015). Recruitment productivity of cod then increased dramatically following the

642 collapse of pelagic fish, potential predators of their early life history stages. This resulted

643 in the rapid recovery of the cod stock due to the production of a series of strong year

644 classes from the mid-1970s to the early 1980s. Cod recovery coincided with declines in the 645 recruitment productivity of hake and plaice, marked by regime shifts in 1979 and 1982, 646 respectively. These shifts in recruitment productivity may result from increased predation

647 by large cod on pre-recruit juveniles of hake and plaice and/or increased competition

648 between these early juveniles and small cod.

650 In our study, we have used bottom trawl survey indices of the spawning stock biomass and 651 number of recruits to avoid any pitfalls related to using stock assessment outputs as data in 652 our analysis (Brooks and Deroba, 2015). However, we did not include any uncertainty 653 estimates of the indices in our analysis but simply assumed that the standard deviation of 654 the log-transformed R/S ratios was proportional to the sampling intensity and inferred the 655 noise parameters from the data. Moreover, the changes in survey gear and protocol in 1985 
656 and vessel in 1985, 1992 and 2004 might have been misinterpreted as regime changes by

657 our method. In general, the identified S-R shifts did not coincide with the timing of survey

658 changes. However, for cod there is a change point (1991) near 1992 in both the Ricker and

659 Beverton-Holt model analyses, for plaice there is a change point (1986) near 1985 in the

660 Beverton-Holt analysis, and for skate there are change points (1988 and 2006) that are

661 close to the survey changes in 1985 and 2005 in both the Ricker and Beverton-Holt

662 analyses. We examined this further, comparing results for thorny skate with and without

663 adjustment for the changes in the survey. (Effects of survey changes were greatest for

664 thorny skate.) The estimated timing of change points was the same using both adjusted and

665 unadjusted survey data (Fig. S7), though the scale of predicted recruit abundance differed,

666 matching the difference in scale between the two data sets (Fig. S8 and S9). In this case at

667 least, change point detection was robust to changes in fishing efficiency in the survey,

668 whether adjusted for or not.

669

670 Earlier studies modelling recruitment regimes have been limited to two regimes (Munch

671 and Kottas 2009, Ottersen et al. 2013), whereas in the BOCPD model the maximum

672 number of regimes does not have an upper bound other than what is imposed by the length

673 of the observation time series. Thus, direct comparison to the previous methods was not

674 possible. It would be interesting, however, to expand the other methods to handle multiple

675 change points, and to explore other methods for modelling time-varying parameters in S-R

676 models for comparison. Here, we assumed that the parameters change suddenly but in

677 some cases, it could be more realistic to assume that the parameters change gradually.

678 
679 Unlike maximum likelihood methods (Ottersen et al. 2013), BOCPD explicitly takes into 680 account uncertainty in both the parameter estimates and the timings of the shifts, and these 681 uncertainties are further propagated into predictions about future recruitment via the 682 posterior predictive distribution. Our method also takes into account the change in the 683 level of noise in the data due to change in sampling intensity, and the method is able to 684 handle years of missing data. Moreover, the earlier studies focused only on the Ricker 685 model, whereas we also applied our method to the Beverton-Holt model. We chose the 686 Ricker and Beverton-Holt S-R models for this study because of their popularity. However, 687 the presented framework easily accommodates any S-R model that can be fitted using 688 sequential Bayesian estimation. Furthermore, we explicitly state our prior assumptions

689 about the residuals in the S-R models, and calculate the posterior distributions of the 690 standard deviation parameters (Fig. S3). Moreover, our model can be also extended to take 691 into account environmental correlates by directly modifying the S-R model to incorporate 692 environmental effects as has been done earlier (e.g. Chen and Irvine 2001). Furthermore, 693 the parameters describing the strengths of the environmental effects can also be assumed 694 piecewise constant as we have done with $\alpha$ and $\beta$ in this study to allow shifts in these 695 parameters.

696

697 For most of the stocks, the posterior median and 68\% central probability interval of the 698 density dependence parameter $\beta$ do not differ much from those of the prior distribution. 699 We have identified two possible reasons for this. Firstly, most of the data points have been 700 collected when the stocks have been at low density and thus there was little information on 701 density dependence in the data. Secondly, the effects of ecosystem regime shifts may be 702 more likely to be transmitted to the S-R process through the $\alpha$ parameter than through the 
$703 \beta$ parameter. The $\beta$ parameter is used to model density-dependent feedbacks on

704 recruitment success (e.g., cannibalism, effects of overcrowding and competition). These

705 effects can certainly be influenced by environmental changes (e.g., decreased abundance

706 of prey for adults, resulting in increased cannibalism; Hjermann et al. 2007). However,

707 direct effects of regime shifts (e.g., of changes in temperature or the abundance of prey,

708 competitors or predators of larvae and early juveniles) would be transmitted to the

709 recruitment process through the $\alpha$ parameter, unless incorporated directly as additional

710 terms in the recruitment model.

711

712 The model can produce a high posterior probability for a shift based on only one

713 observation that is not well explained by the posterior predictive distribution of the

714 previous regime. However, the posterior probabilities cannot tell if the latest observation is

715 the first observation from a new regime or an outlier produced by e.g. sampling error.

716 When more data is collected in the coming years, the smoothed run length probabilities

717 will retrospectively show which was the case. An outlier would be identified by finding a

718 huge discrepancy between the posterior and smoothed probabilities of a new run in the

719 particular year. In addition, even if the new observation actually comes from a new regime,

720 the regime-specific parameter estimates of the S-R relationship have to be very uncertain

721 since they are based only on one observation, and are heavily affected by the prior

722 distribution of the parameters. As an example of this, we point to the last two years in the

723 case of cod. Although the shift in 2013 seems obvious based on the smoothed run length

724 probabilities (Fig. 3), the posterior parameter estimates (Fig. 4), and by just looking at the

725 data (Fig. 6), it is highly questionable that the maximum recruit production per unit of

726 spawning stock biomass in 2014 is really more than eight times what it was in 2012. It 
727 may well be that in both 2013 and 2014 surveys the recruitment was overestimated and/or

728 the spawning biomass underestimated. However, the small amount of data available for the

729 regime starting in 2013 results in high uncertainty in the carrying capacity (Fig. 5), and a

730 higher standard deviation (Fig. S3).

731

732 Even though the BOCPD modelling framework is theoretically sound, additional evidence

733 of the inferred shifts may be seen in the tendency of the constant parameter model

734 estimates to approach the BOCPD model estimates after a shift. This gives us even more

735 confidence stating that recruitment shifts have occurred for the stocks examined in this

736 study, and also that there is little reason to assume that shifts in S-R relationship would not

737 also happen in the future.

738

739 The assumption of time-invariant parameters is common when modelling complex

740 biological systems. However, as pointed out in this study, some parameters may well be

741 constant within some limited time range, but experience sudden and relatively strong

742 changes. In these cases, using constant model parameters may lead to serious over- or

743 underestimation of key population parameters and their uncertainty, subsequently resulting

744 in erroneous conclusions about the status of the population and forecasts of its future

745 development.

746

747 The stock-recruitment relationship plays a critical role in determining fisheries

748 management targets and limits (e.g., Shepherd 1982) and in population projections of

749 future biomass. Thus, the scientific advice provided to fisheries management would be

750 expected to be improved if it took into account effects of regime shifts on stocks (e.g., 
751 King et al. 2015). However, success depends on the ability to reliably identify the timing

752 and extent of regime shifts (e.g., Basson 1999, King and McFarlane 2006). One approach

753 has been to link a component of productivity (e.g., recruitment) to an environmental

754 variable such as temperature and make projections or estimate reference points based on

755 this relationship (Punt et al. 2014). Pitfalls of this approach include the possibility that

756 these relationships are spurious, break down over time or change over time. A second

757 approach attempts to identify regimes based on deviations (e.g., in annual recruitment)

758 from the previous average value (e.g., STARS, Rodionov and Overland 2005) or

759 potentially from model predictions (e.g., BOCPD).

760

761 The utility of incorporating information on recruitment regimes in fisheries management

762 can be examined using Management Strategy Evaluation (MSE; Punt et al. 2014). In many

763 cases evaluated using MSE, incorporating effects of regime shifts in management

764 procedures failed to improve management performance (Punt et al. 2014; King et al.

765 2015). Reasons included high uncertainty in environmental and recruitment measurements

766 (King et al. 2015), failure to detect regimes when they were present or detecting them

767 when they were not (e.g., A'mar et al. 2009), and misidentifying regime timing combined

768 with noisy estimates of management reference points due to the reduced sample size

769 within regimes (Szuwalski and Punt 2013). However, use of BOCPD as the algorithm to

770 detect regime shifts may improve performance due to its robust accounting of uncertainty

771 and its probabilistic designation of regime shifts. Until information on regime shifts can be

772 more reliably integrated directly into stock assessment models and estimation of biological

773 reference points, King et al. (2015) suggest using this information in a supporting role. For

774 example, biomass projections could be made assuming below average, average and above 
775

776

777

778

779

780

781

782

783

784

785

786

787

788

789

790

791

792

793

794

795

796

797

798

average recruitment rates and advice based on these scenarios could be summarized along with associated risks. In this case, BOCPD could be used to assign relative probabilities to the various scenarios.

As pointed out by many fisheries scientists, establishing S-R relationships that are useful in fisheries sciences is a difficult endeavor (Hilborn and Walters 1992, Subbey at al. 2014). As the present study illustrates, incorporating the assumption of recruitment regimes in the S-R models will bring us a step closer to understanding drivers of recruitment. However, while recruitment is one of the most essential aspects of fish population dynamics, other parts of the population dynamics could also experience shifts in the sense described in this paper. Bayesian online change point detection offers a theoretically sound modular framework for regime shift modelling to be used with any kind of a model relevant to fish population dynamics.

\section{Supplementary material}

The following supplementary material is available on the journal Web site. Section A:

Derivations of the recursive computation of the run length posterior distribution and the full posterior distribution of the parameters; Section B: Description of the prior sensitivity analyses; Section C: Additional figures; Section D: Additional tables.

\section{Acknowledgements}

This study was funded by the Academy of Finland (through a grant to AK) and by the National Science and Engineering Research Council of Canada (through a disvovery grant to AK). The authors would also like to thank anonymous referees for helpful comments. 


\section{References}

800 Adams, R. P., and MacKay, D. J. C. 2007. Bayesian online changepoint detection.

801 Technical report, University of Cambridge, Cambridge, UK.

802 Alheit, J., Möllmann, C., Dutz, J., Kornilovs, G., Loewe, P., Mohrholz, V., and Wasmund,

803 N. 2005. Synchronous ecological regime shifts in the central Baltic and the North

804 Sea in the late 1980s. ICES Journal of Marine Science: Journal du Conseil, 62(7):

$805 \quad 1205-1215$.

806 A'mar, Z. T., Punt, A. E., and Dorn, M. W. 2009. The impact of regime shifts on the

807 performance of management strategies for the Gulf of Alaska walleye pollock

808 fishery. Canadian Journal of Fisheries and Aquatic Sciences, 66(12): 2222-2242.

809 Basson, M. 1999. The importance of environmental factors in the design of management

$810 \quad$ procedures. ICES Journal of Marine Science: Journal du Conseil, 56(6):

$811 \quad 933-942$.

812 Beamish, R. J., Benson, A.J., Sweeting, R.M., and Neville, C. M. 2004. Regimes and the

813 history of the major fisheries off Canada's west coast. Progress in Oceanography,

60(2): $355-385$.

815 Beaugrand, G. 2004. The North Sea regime shift: evidence, causes, mechanisms and

816 consequences. Progress in Oceanography, 60(2): 245-262.

817 Begg, G. A., and Marteinsdottir, G. 2002. Environmental and stock effects on spawning

818 origins and recruitment of cod Gadus morhua. Marine Ecology Progress Series,

$819 \quad 229: 263-277$.

820 Benoit, H. P. 2006. Standardizing the southern Gulf of St. Lawrence bottom-trawl survey

821 time series: results of the 2004-2005 comparative fishing experiments and other 
822

823

824

825

826

827

828

830

831

832

833

834

836

837

838

839

840

841

842

844

recommendations for the analysis of the survey data. DFO Canadian Science Advisory Secretariat Research Document, 2006/008.

Benoît, H. P., and Swain, D. P. 2003a. Standardizing the southern Gulf of St. Lawrence bottom-trawl survey time series: adjusting for changes in research vessel, gear and survey protocol. Canadian Technical Report of Fisheries and Aquatic Sciences, $2505,95 \mathrm{pp}$.

Benoît, H. P., and Swain, D. P. 2003b. Accounting for length and depth-dependent diel variation in catchability of fish and invertebrates in an annual bottom-trawl survey. ICES Journal of Marine Science, 60: 1298-1317.

Benoit, H. P., and Swain, D. P. 2008. Impacts of environmental change and direct and indirect harvesting effects on the dynamics of a marine fish community. Canadian Journal of Fisheries and Aquatic Sciences, 65(10): 2088-2104.

Beverton, R. J. H., and Holt, S. J. 1957. On the dynamics of exploited fish populations. Fisheries Investigation Series 2, volume 19, UK Ministry of Agriculture. Fisheries, and Food, London, UK.

Brooks, E. N., and Deroba, J. J. 2015. When "data" are not data: the pitfalls of post-hoc analyses that use stock assessment model output. Canadian Journal of Fisheries and Aquatic Sciences, 72(4), 634-641.

Brunel, T., and Boucher, J. 2007. Long-term trends in fish recruitment in the north-east Atlantic related to climate change. Fisheries Oceanography, 16: 336-349.

Chen, D. G., and Irvine, J. R. 2001. A semiparametric model to examine stock recruitment relationships incorporating environmental data. Canadian Journal of Fisheries and Aquatic Sciences, 58(6): 1178-1186. 
845 Ciannelli, L., Chan, K. S., Bailey, K. M., and Stenseth, N. C. 2004. Nonadditive effects of

846 the environment on the survival of a large marine fish population. Ecology, 85(12),

$847 \quad 3418-3427$.

848 deYoung, B., Barange, M., Beaugrand, G., Harris, R., Perry, R. I., Scheffer, M., and

849 Werner, F. 2008. Regime shifts in marine ecosystems: detection, prediction and

$850 \quad$ management. Trends in Ecology and Evolution, 23(7): 402-409.

851 Fauchald, P. 2010. Predator-prey reversal: a possible mechanism for ecosystem hysteresis

$852 \quad$ in the North Sea? Ecology, 91(8): 2191-2197.

853 Hilborn, R. 2005. Are sustainable fisheries achievable? In Marine conservation biology:

854 the science of maintaining the sea's biodiversity. pp. 247-259. Island Press,

$855 \quad$ Washington, DC.

856 Hilborn, R., and Walters, C. J. 1992. Quantitative fisheries stock assessment: choice,

857 dynamics and uncertainty. Reviews in Fish Biology and Fisheries, 2(2): 177-178.

858 Hjermann, D. Ø., Bogstad, B., Eikeset, A. M., Ottersen, G., Gjøsæter, H., and Stenseth, N.

859 C. 2007. Food web dynamics affect Northeast Arctic cod recruitment. Proceedings

$860 \quad$ of the Royal Society of London B: Biological Sciences, 274(1610), 661-669.

861 Hjort, J. 1914. Fluctuations in the great fisheries of northern Europe viewed in the light of

862 biological research. ICES.

863 Jeffreys, H. 1946. An invariant form for the prior probability in estimation problems. In

864 Proceedings of the Royal Society of London A: Mathematical, Physical and

$865 \quad$ Engineering Sciences, 186(1007), 453-461.

866 Jiao Y. 2009. Regime shift in marine ecosystems and implications for fisheries

867 management, a review. Rev. Fish Biol. Fish., 19, 177-191. 
868 King, J. R., and McFarlane, G. A. 2006. A framework for incorporating climate regime 869 shifts into the management of marine resources. Fisheries Management and $870 \quad$ Ecology, 13(2): 93-102.

871 King, J. R., McFarlane, G. A., and Punt A. E. 2015. Shifts in fisheries management: adapting to regime shifts. Philosophical Transactions of the Royal Society B: Biological Sciences, 370(1659).

Liu, J., and West, M. 2001. Combined parameter and state estimation in simulation-based filtering. In Sequential Monte Carlo methods in practice pp. 197-223. Springer

Millar, R. B. 2002. Reference priors for Bayesian fisheries models. Canadian Journal of Fisheries and Aquatic Sciences, 59(9), 1492-1502.

Morin, R., Swain, D. P., and LeBlanc, S. G. 2013. A biomass Limit Reference Point for the NAFO 4T American plaice (Hippoglossoides platessoides) fishery. DFO Canadian Science Advisory Secretariat Research Document. 2012/108. ii + 37 p.

Möllmann, C., Müller-Karulis, B., Kornilovs, G., and St John, M. A. 2008. Effects of climate and overfishing on zooplankton dynamics and ecosystem structure: regime shifts, trophic cascade, and feeback loops in a simple ecosystem. ICES Journal of Marine Science, 65(3): 302-310

Möllmann, C., Folke, C., Edwards, M., and Conversi, A. 2015. Marine regime shifts around the globe: theory, drivers and impacts. Phil. Trans. R. Soc. B, 370(1659).

Munch, S. B., and Kottas, A. 2009. A Bayesian modeling approach for determining 889 productivity regimes and their characteristics. Ecological Applications, 19(2):

890 $527-537$. 
891 Needle, C. L. 2001. Recruitment models: diagnosis and prognosis. Reviews in Fish Biology $892 \quad$ and Fisheries, 11(2): 95-111.

893 Okamoto, D. K., Schmitt, R. J., Holbrook, S. J., and Reed, D. C. 2012. Fluctuations in 894 food supply drive recruitment variation in a marine fish. Proceedings of the Royal $895 \quad$ Society of London, Series B, 279: 4542-4550.

896 Olsen, E. M., Ottersen, G., Llope, M., Chan, K-S., Beaugrand, G., and Stenseth, N-C.

897 2011. Spawning stock and recruitment in North Sea cod shaped by food and

898 climate. Proceedings of the Royal Society of London, Series B, 278: 504-510.

899 Ottersen, G., Stige, L. C., Durant, J. M., Chan, K. S., Rouyer, T. A., Drinkwater, K. F., and 900 Stenseth, N. C. 2013. Temporal shifts in recruitment dynamics of North Atlantic 901 fish stocks: effects of spawning stock and temperature. Marine Ecology Progress

$902 \quad$ Series, 480: 205-225.

903 Perälä, T., and Kuparinen, A. 2015. Detecting regime shifts in fish stock dynamics.

$904 \quad$ Canadian Journal of Fisheries and Aquatic Sciences, 72(11): 1619-1628.

905 Punt, A. E., A’mar, T., Bond, N. A., Butterworth, D. S., de Moor, C. L., De Oliveira, J. A.

906 A., Haltuch, M. A., Hollowed, A. B., and Szuwalski, C. 2014. Fisheries

907 management under climate and environmental uncertainty: control rules and

908 performance simulation. ICES Journal of Marine Science: Journal du Conseil, $909 \quad 71(8): 2208-2220$.

910 Ricker, W. E. 1954. Stock and recruitment. Journal of the Fisheries Board of Canada, $911 \quad 11(5): 559-623$.

912 Rodionov, S., and Overland, J. E. 2005. Application of a sequential regime shift detection 913 method to the Bering Sea ecosystem. ICES Journal of Marine Science: Journal du $914 \quad$ Conseil, 62(3): 328-332. 
915 Särkkä, S. 2013. Bayesian filtering and smoothing. Cambridge University Press.

916 Shepherd, J. G. 1982. A versatile new stock-recruitment relationship for fisheries, and the

917 construction of sustainable yield curves. Journal du Conseil, 40: 67-75.

918 Steele, J. H. 1996. Regime shifts in fisheries management. Fisheries Research, 25(1):

$919 \quad 19-23$.

920 Stige, L. C., Hunsicker, M. E., Bailey, K. M., Yaragina, N. A., and Hunt Jr, G. L. 2013.

921 Predicting fish recruitment from juvenile abundance and environmental indices.

922 Marine Ecology Progress Series, 480: 245-261.

923 Subbey, S., Devine, J. A., Schaarschmidt, U., and Nash, R. D. 2014. Modelling and

924 forecasting stock-recruitment: current and future perspectives. ICES Journal of

925 Marine Science: Journal du Conseil, 71(8): 2307-2322.

926 Swain, D.P., and Benoît, H.P. 2015. Extreme increases in natural mortality prevent

927 recovery of collapsed fish populations in a Northwest Atlantic ecosystem. Marine

$928 \quad$ Ecology Progress Series, 519: 165-182.

929 Swain, D. P., and Sinclair, A. F. 2000. Pelagic fishes and the cod recruitment dilemma in

930 the Northwest Atlantic. Canadian Journal of Fisheries and Aquatic Sciences, 57:

$931 \quad 1321-1325$.

932 Swain, D. P., Jonsen, I. D., Simon, J. E. and Davies, T. D. 2013. Contrasting decadal

933 trends in mortality between large and small individuals in skate populations in

934 Atlantic Canada. Canadian Journal of Fisheries and Aquatic Sciences, 70(1):

$935 \quad 74-89$.

936 Swain, D.P., Savoie, L., and Cox, S.P. 2016. Recovery potential assessment of the

937 Southern Gulf of St. Lawrence Designatable Unit of White Hake (Urophycis tenuis 

Mitchill), January 2015. DFO Can. Sci. Advis. Sec. Res. Doc. 2016/045.

939 vii +109 p.

940 Szuwalski, C. S., and Punt, A. E. 2013. Fisheries management for regime-based ecosystems: a management strategy evaluation for the snow crab fishery in the eastern Bering Sea. ICES Journal of Marine Science: Journal du Conseil, 70(5): $955-967$.

944 Szuwalski, C. S., Vert-Pre, K. A., Punt, A. E., Branch, T. A., and Hilborn, R. 2014.

945 Examining common assumptions about recruitment: a meta-analysis of recruitment 946 dynamics for worldwide marine fisheries. Fish and Fisheries.

947 Vasilakopoulos, P., and Marshall, C. T. 2015. Resilience and tipping points of an exploited

948 fish population over six decades. Global change biology, 21(5): 1834-1847.

949 Vert-pre, K.A., Amoroso, R.O., Jensen, O.P., and Hilborn, R. 2013. Frequency and 950 intensity of productivity regime shifts in marine fish stocks. Proceedings of the $951 \quad$ National Academy of Sciences. 110(5): 1779-1784.

952 
953 Table 1. Model parameters for the study species: Atlantic cod (Gadus morhua), white

954 hake (Urophycis tenuis), American plaice (Hippoglossoides platessoides), and thorny skate

955 (Amblyraja radiata).

\begin{tabular}{l|c|c|c|c|c|c|c|c|c|c|c|c|c|} 
Species & $a_{R}$ & $\alpha_{\mathrm{low}}^{\mathrm{R}}$ & $\alpha_{\mathrm{up}}^{\mathrm{R}}$ & $\beta_{\mathrm{low}}^{\mathrm{R}}$ & $\beta_{\mathrm{up}}^{\mathrm{R}}$ & $\sigma_{\mathrm{low}}^{\mathrm{R}}$ & $\sigma_{\mathrm{up}}^{\mathrm{R}}$ & $\alpha_{\mathrm{low}}^{\mathrm{BH}}$ & $\alpha_{\mathrm{up}}^{\mathrm{BH}}$ & $\beta_{\mathrm{low}}^{\mathrm{BH}}$ & $\beta_{\mathrm{up}}^{\mathrm{BH}}$ & $\sigma_{\mathrm{low}}^{\mathrm{BH}}$ & $\sigma_{\mathrm{up}}^{\mathrm{BH}}$ \\
\hline \hline cod & 3 & 0.1 & 4 & $5 \cdot 10^{-9}$ & $10 \cdot 10^{-9}$ & 0.3 & 1.5 & 0.1 & 4 & $10 \cdot 10^{-9}$ & $20 \cdot 10^{-9}$ & 0.3 & 1.5 \\
\hline hake & 2 & 0.1 & 6 & $30 \cdot 10^{-9}$ & $200 \cdot 10^{-9}$ & 0.3 & 1.5 & 0.1 & 6 & $60 \cdot 10^{-9}$ & $400 \cdot 10^{-9}$ & 0.3 & 1.5 \\
\hline plaice & 4 & 0.2 & 6 & $10 \cdot 10^{-9}$ & $20 \cdot 10^{-9}$ & 0.3 & 1.5 & 0.2 & 6 & $20 \cdot 10^{-9}$ & $40 \cdot 10^{-9}$ & 0.3 & 1.5 \\
\hline skate & 2 & 0.1 & 4 & $400 \cdot 10^{-9}$ & $2000 \cdot 10^{-9}$ & 0.3 & 1.5 & 0.1 & 4 & $800 \cdot 10^{-9}$ & $4000 \cdot 10^{-9}$ & 0.3 & 1.5
\end{tabular}

956

957 Table 2. RMSE of the posterior median estimates of parameters $(\widehat{\alpha}, \widehat{\beta}$ and $\widehat{\sigma})$, derived

958 quantities $\left(\widehat{R_{\infty}}\right.$ and $\left.\widehat{K}\right)$, and the median of the posterior predictive distribution $\left(\widehat{R_{t+1}}\right)$ for

959 different noise levels $(\sigma \in\{0.3,0.4,0.5,0.6\})$ for the change point model and constant

960 model. The three reported numbers are the $16^{\text {th }}$ percentile, the median, and the $84^{\text {th }}$

961 percentile, respectively. The units for $\widehat{\beta}$ are $\frac{10^{-9}}{\mathrm{~kg}}$, and for $\widehat{R_{t+1}}$ they are $10^{6}$ of

962 individuals.

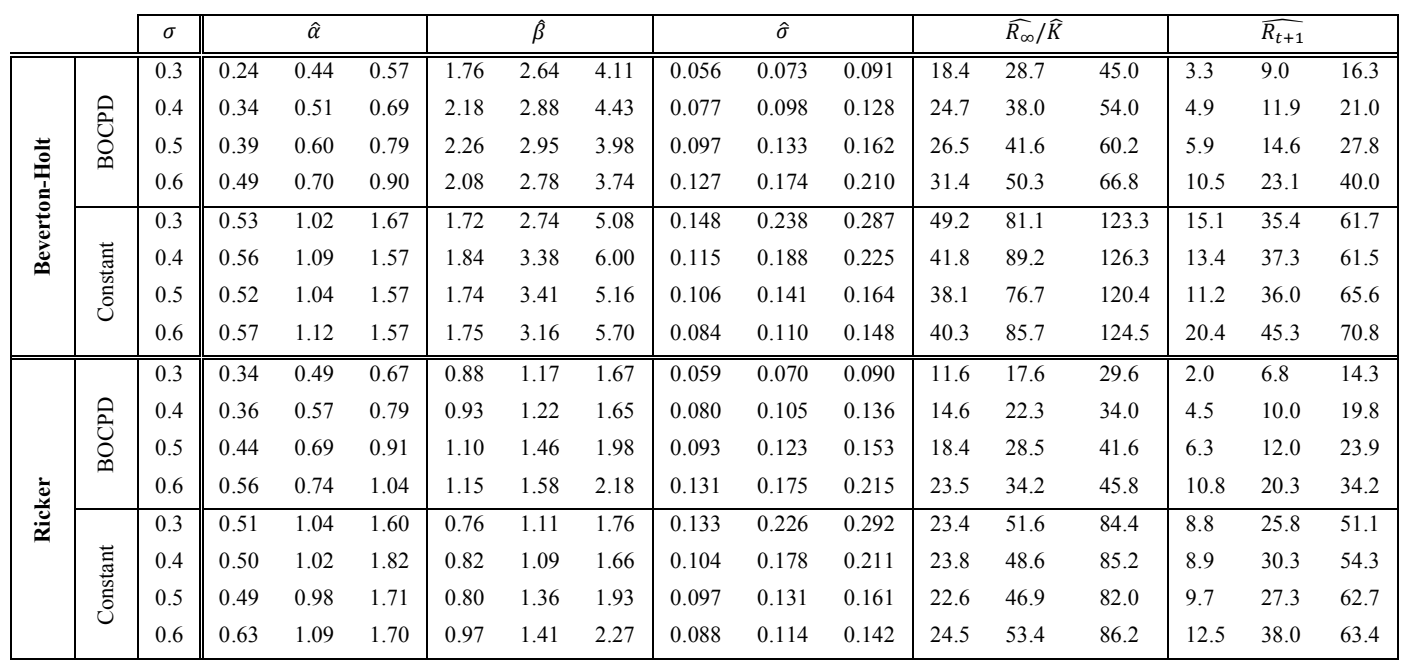

963

964 


\section{Figure legends}

966

967 Figure 1. The results of the change point detection accuracy in the Beverton-Holt model

968 simulations for the four noise levels $(\sigma \in\{0.3,0.4,0.5,0.6\})$. The vertical axis shows the

969 number of change points and the horizontal axis shows the absolute change in $\alpha^{\mathrm{BH}}$

970 parameter before and after the change point. Black color denotes the change points that

971 were inferred correctly, gray color denotes those change points that were detected but with

972 an error of \pm 1 or \pm 2 time steps, and white denotes the rest of the change points that we

973 considered as missed by our method. The corresponding figure for the Ricker model

974 simulations is found in the supplementary material.

975

976 Figure 2. Empirical cumulative distribution functions illustrating the accuracy of the

977 parameter estimates in the Beverton-Holt model simulations. The vertical axis shows the

978 proportion of time steps within a simulation case for which the true parameter value was

979 within the posterior central probability interval (CPI), whereas the horizontal line shows

980 the proportion of all the simulation cases. The dashed line denotes the BOCPD model and

981 the solid line denotes the model with time-invariant parameters. Different noise levels

$982(\sigma \in\{0.3,0.4,0.5,0.6\})$ are denoted with increasing darkness of the curves. The originally

983 staircase-like curves were smoothed to clarify patterns.

985 Figure 3. The smoothed run length probability distributions for the study species: Atlantic

$986 \operatorname{cod}$ (Gadus morhua), white hake (Urophycos tenuis), American plaice (Hippoglossoides

987 platessoides) and thorny skate (Amblyraja radiata). The left and right column show the

988 run length probabilities when the UPM is the Ricker model and Beverton-Holt model, 
989 respectively. The red lines show the most likely segmentation of the data based on the 990 smoothed run length probabilities.

991

992 Figure 4. The sequential update of the posterior marginal densities of the maximum 993 number of recruits per unit of spawning stock biomass for the study species. Output for the

994 Ricker $\left(\alpha^{\mathrm{R}}\right)$ and Beverton-Holt $\left(\alpha^{\mathrm{BH}}\right)$ models are shown in left and right columns,

995 respectively. The colored solid lines denote the posterior median and the shaded areas

996 indicate the $68 \%$ central posterior probability interval. The regimes as identified using the

997 most likely segmentation of the data are denoted by the change in the color of the shading.

998 The black lines indicate the posterior median and $68 \%$ posterior probability interval of the

999 constant parameter model. The supplementary material includes figures for the posterior

1000 marginal densities of the density dependence parameter $\beta$ (Fig. S3), and the standard

1001 deviation parameter $\delta_{t}$ (Fig. S4).

1002

1003 Figure 5. The sequential update of the posterior marginal densities of the carrying capacity

1004 for recruits $k=\frac{\alpha^{\mathrm{R}}}{e \beta^{\mathrm{R}}}$ in the Ricker model and the asymptotic maximum number of recruits

$1005 R_{\infty}=\frac{\alpha^{\mathrm{BH}}}{\beta^{\mathrm{BH}}}$ in the Beverton-Holt model. Medians and $68 \%$ central probability intervals are

1006 shown. Regimes are denoted with different colors. Constant parameter model fit is shown 1007 in black.

1008

1009 Figure 6. Medians of the posterior predictive distributions of $R_{t}$ for the Ricker and

1010 Beverton-Holt S-R models using BOCPD (solid colored lines) and the constant parameter 
1011 model (dashed black line). The data points belonging to each regime are colored using the 1012 same color as the median lines.

1013 
1014 Fig. 1
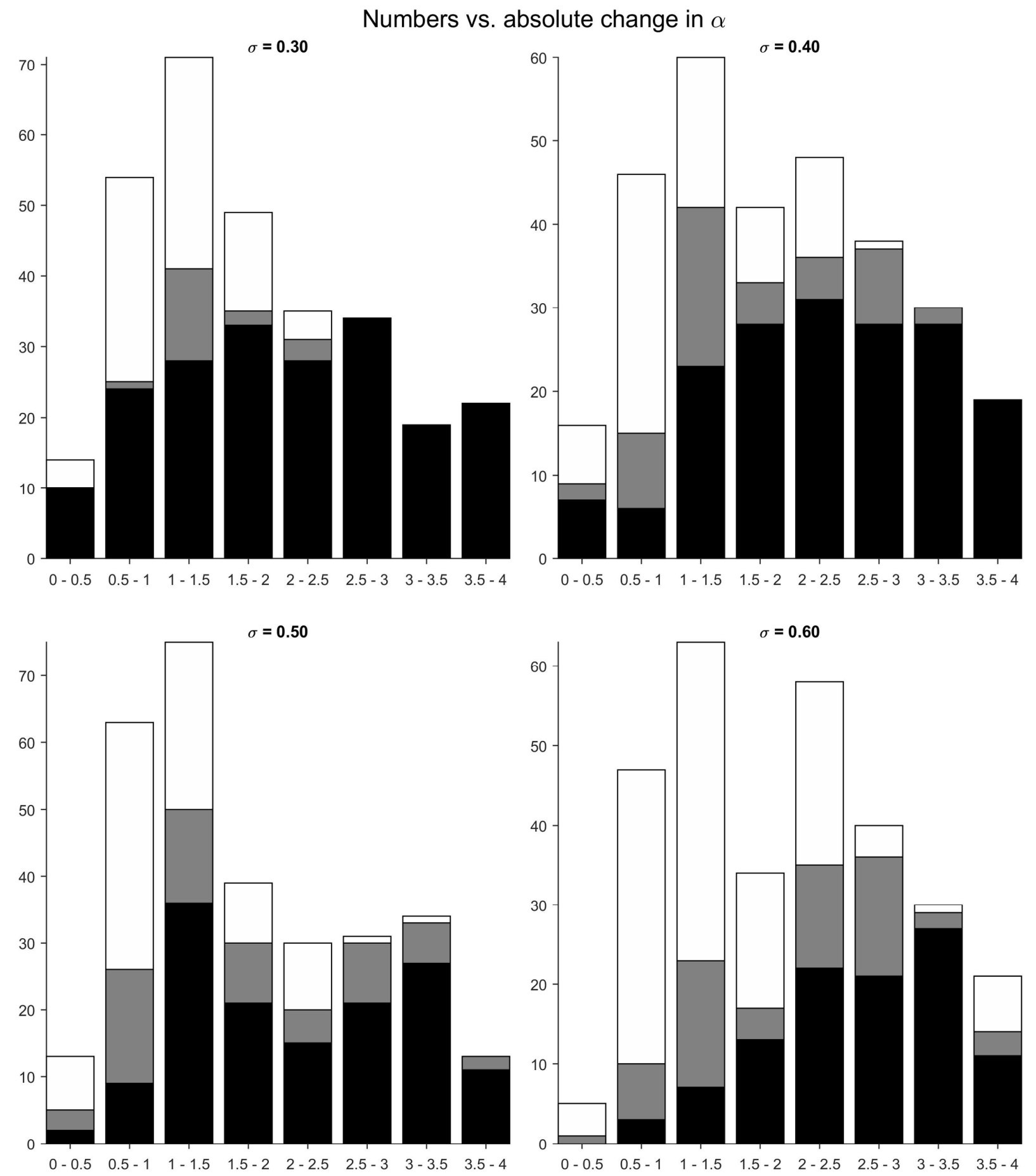

1015

1016 
1017 Fig. 2
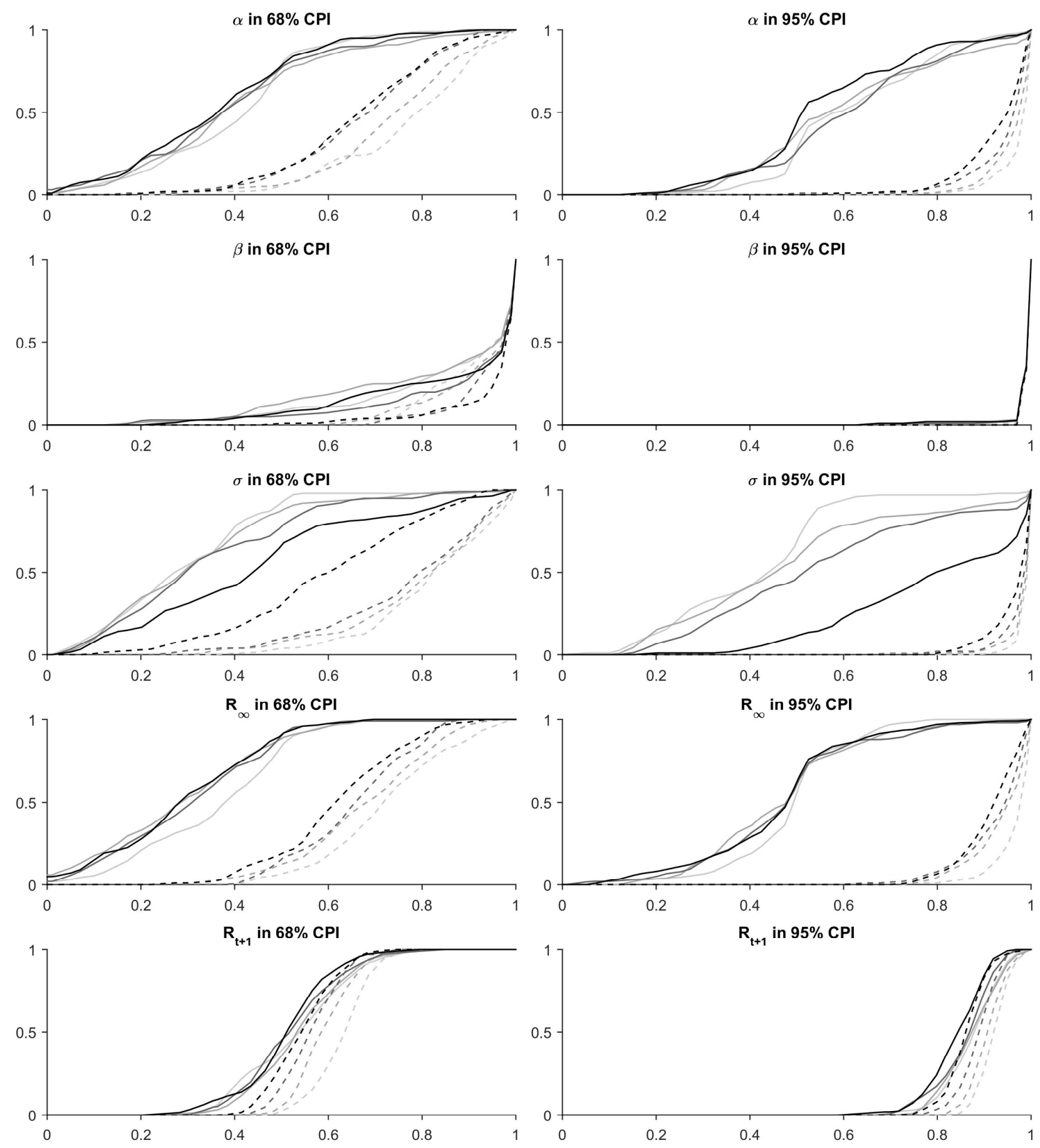

1018

1019 
1020 Fig. 3

1021

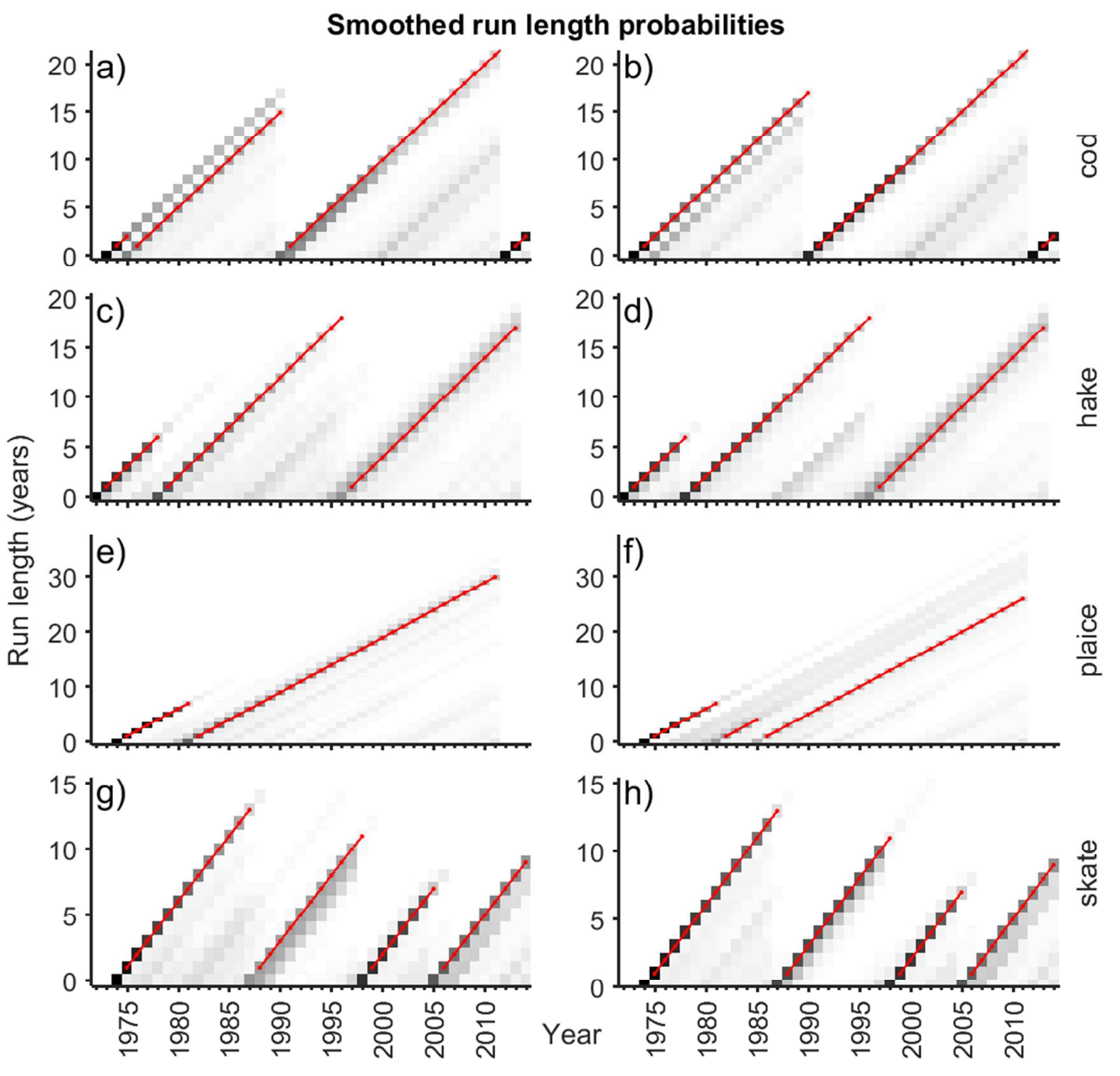

1022 

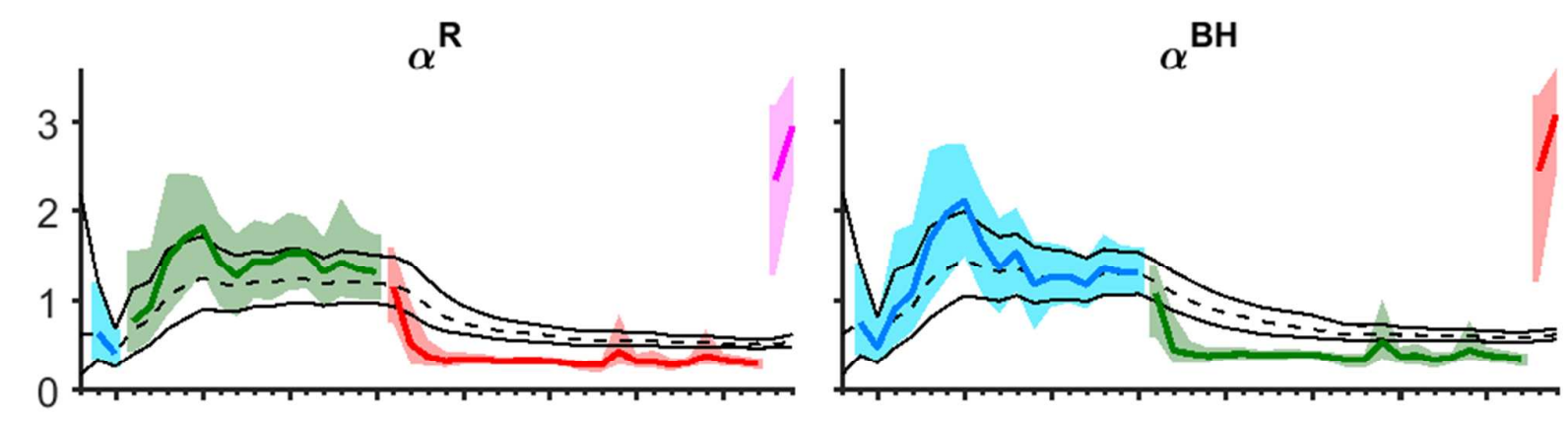

응
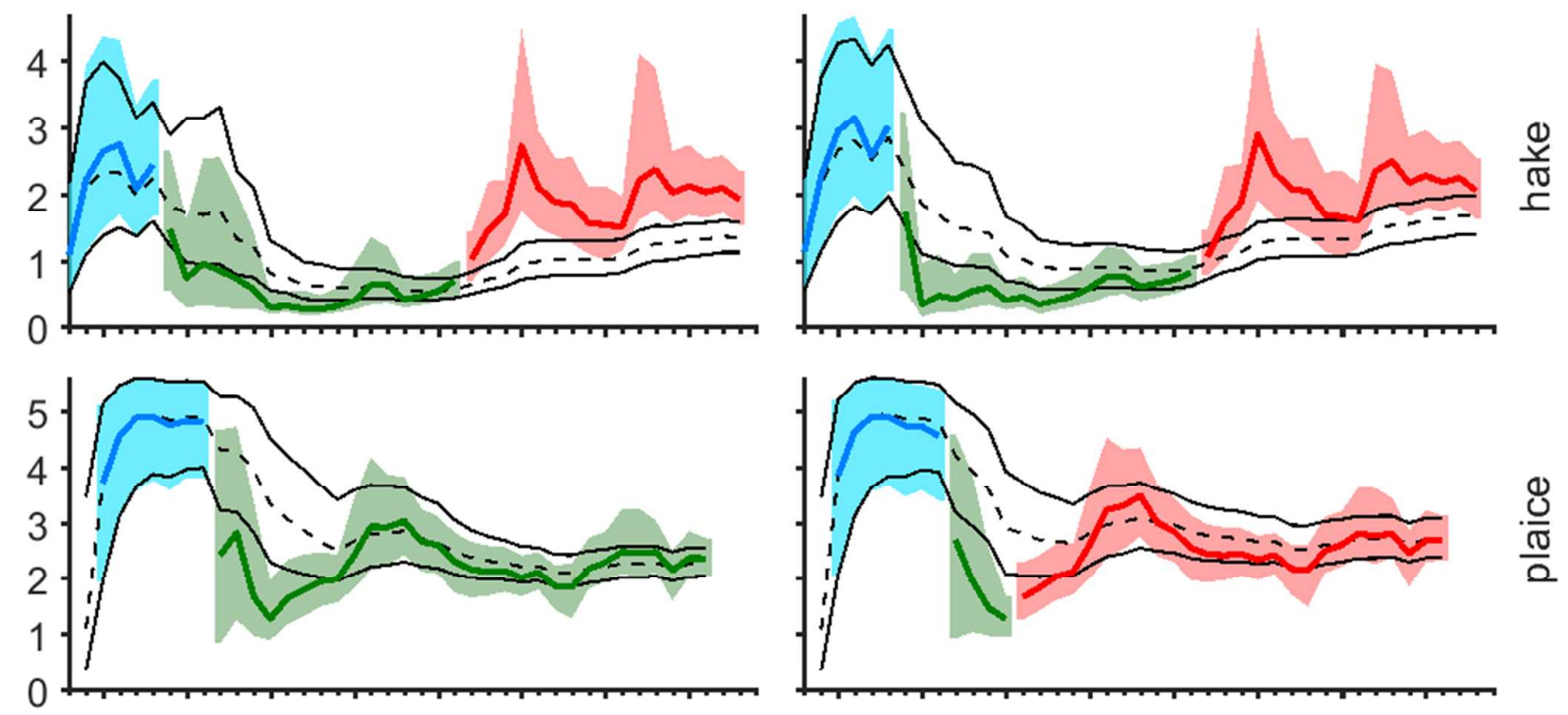

1024

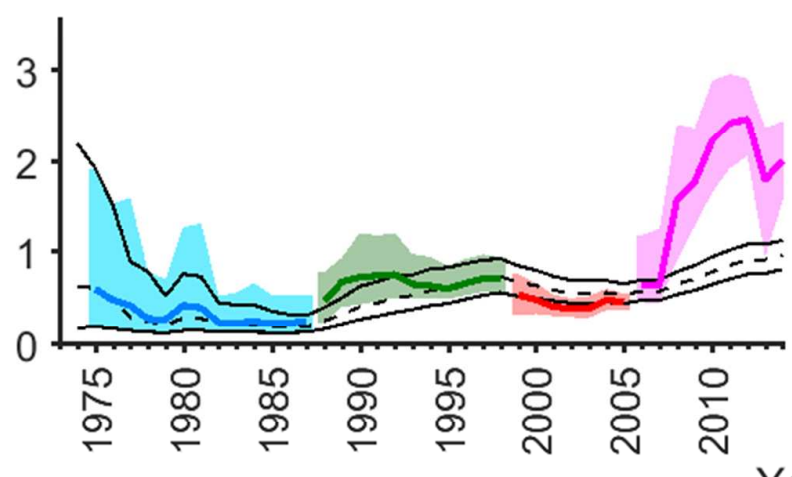

1025 

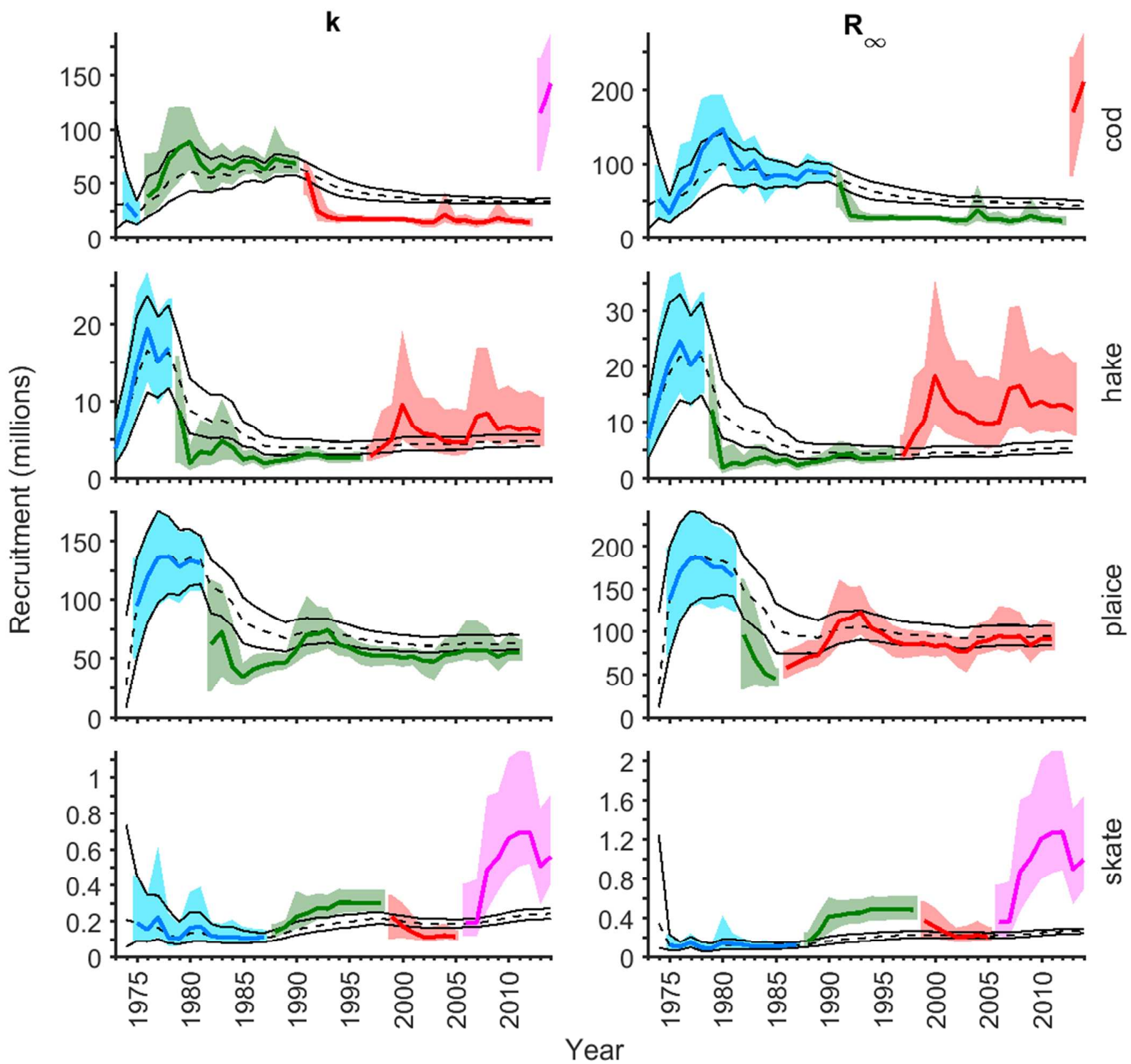

1027 
1028 Fig. 6

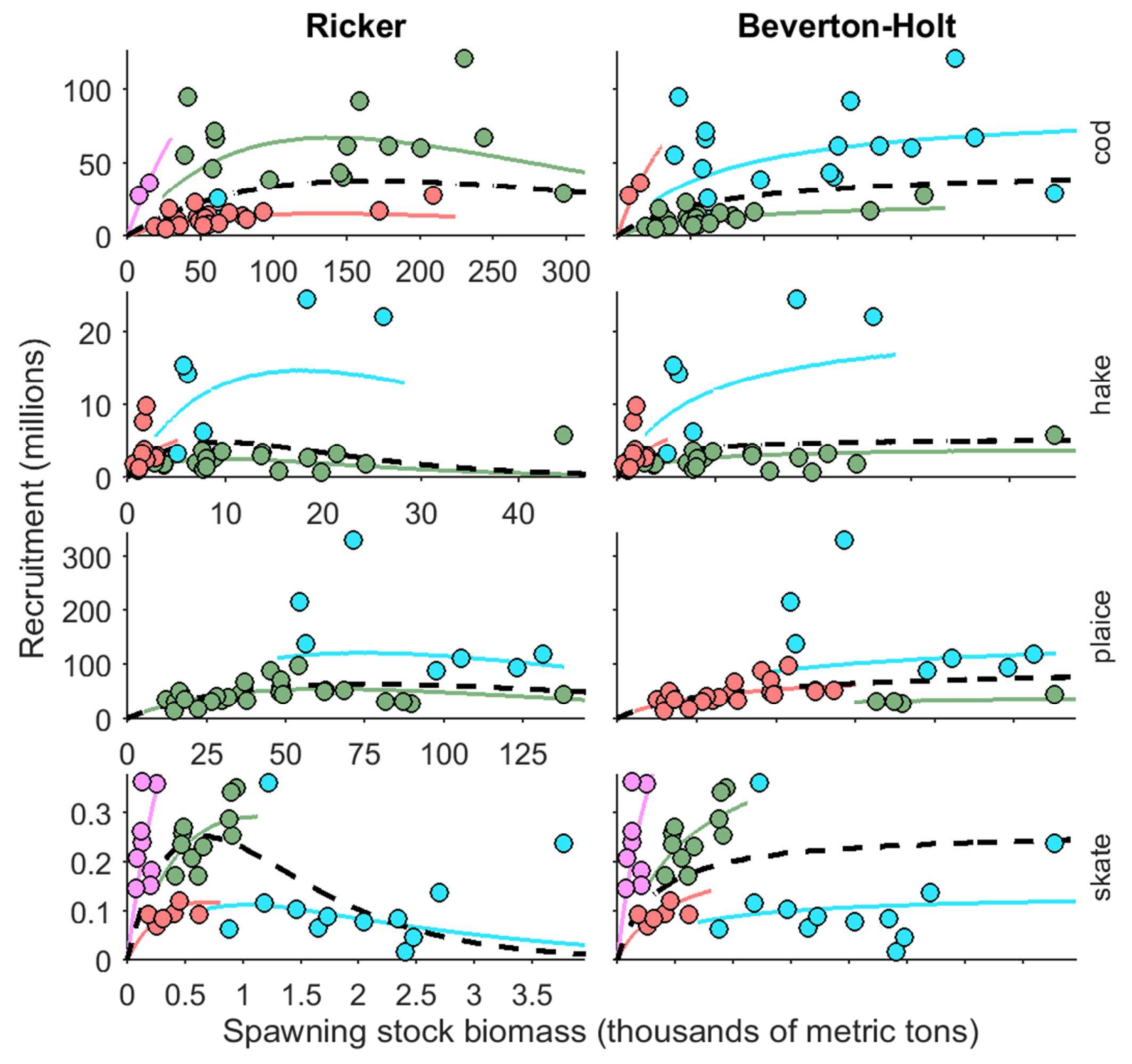

1029 


\section{Examining non-stationarity in the recruitment}

2 dynamics of fishes using Bayesian change point

3 analysis

$4 \quad$ Supplementary material

5 Tommi A. Perälä ${ }^{*}$, Douglas P. Swain ${ }^{2}$, Anna Kuparinen ${ }^{1}$,

6

$7{ }^{1}$ Department of Environmental Sciences, P. O. Box 65, 00014 University of Helsinki,

8 Finland

$9{ }^{2}$ Department of Fisheries and Oceans Canada, Gulf Fisheries Centre, P.O. Box 5030,

10 Moncton, NB E1C 9B6, Canada

$11 *$ Corresponding author: tommi.perala@helsinki.fi 


\section{Section A: Derivations}

13 The recursive computation of the run length posterior distribution

$$
\begin{aligned}
& p\left(r_{t} \mid y_{1: t}, S_{1: t}\right)=\frac{p\left(r_{t}, y_{1: t} \mid S_{1: t}\right)}{p\left(y_{1: t} \mid S_{1: t}\right)}=\frac{1}{p\left(y_{1: t} \mid S_{1: t}\right)} \sum_{r_{t-1}=0}^{t-1} p\left(r_{t}, r_{t-1}, y_{t}, y_{1: t-1} \mid S_{t}\right) \\
& =\frac{1}{p\left(y_{1: t} \mid S_{1: t}\right)} \sum_{r_{t-1}=0}^{t-1} p\left(r_{t}, y_{t} \mid r_{t-1}, y_{1: t-1}, S_{1: t}\right) p\left(r_{t-1}, y_{1: t-1} \mid S_{1: t}\right) \\
& =\frac{1}{p\left(y_{1: t} \mid S_{1: t}\right)} \sum_{r_{t-1}=0}^{t-1} p\left(r_{t} \mid r_{t-1}, y_{1: t-1}, S_{1: t}\right) p\left(y_{t} \mid r_{t-1}, y_{1: t-1}, S_{1: t}\right) p\left(r_{t-1}, y_{1: t-1} \mid S_{1: t-1}\right) \\
& =\frac{1}{p\left(y_{1: t} \mid S_{1: t}\right)} \sum_{r_{t-1}=0}^{t-1} p\left(r_{t} \mid r_{t-1}\right) p\left(y_{t} \mid y_{\left(t-r_{t-1}\right):(t-1)}, S_{\left(t-r_{t-1}\right):(t-1)}\right) p\left(r_{t-1}, y_{1: t-1} \mid S_{1: t-1}\right) \\
& =\sum_{r_{t-1}=0}^{t-1} \frac{p\left(r_{t} \mid r_{t-1}\right) p\left(y_{t} \mid y_{t-1}^{\left(r_{t-1}\right)}, S_{t-1}^{\left(r_{t-1}\right)}\right) p\left(r_{t-1} \mid y_{1: t-1}, S_{1: t-1}\right) p\left(y_{1: t-1} \mid S_{1: t-1}\right)}{p\left(y_{1: t} \mid S_{1: t}\right)} \\
& =\sum_{r_{t-1}=0}^{t-1} \frac{p\left(r_{t} \mid r_{t-1}\right) p\left(y_{t} \mid y_{t-1}^{\left(r_{t-1}\right)}, S_{t-1}^{\left(r_{t-1}\right)}\right) p\left(r_{t-1} \mid y_{1: t-1}, S_{1: t-1}\right)}{p\left(y_{t} \mid y_{1: t-1}, S_{1: t-1}, S_{t}\right)}
\end{aligned}
$$


15 The posterior density function of the parameters

$$
\begin{aligned}
p\left(\alpha, \beta, \sigma \mid y_{1: t}, S_{1: t}\right) & =\sum_{r_{t}=1}^{t} p\left(\alpha, \beta, \sigma, r_{t} \mid y_{1: t}, S_{1: t}\right) \\
& =\sum_{r_{t}=1}^{t} p\left(r_{t} \mid y_{1: t}, S_{1: t}, r_{t}>0\right) p\left(\alpha, \beta, \sigma \mid r_{t}, y_{1: t}, S_{1: t}\right) \\
& =\sum_{r_{t}=1}^{t} p\left(r_{t} \mid y_{1: t}, S_{1: t}, r_{t}>0\right) p\left(\alpha, \beta, \sigma \mid y_{\left(t-r_{t}+1\right): t}, S_{\left(t-r_{t}+1\right): t}\right) \\
& =\sum_{r_{t}=1}^{t} p\left(r_{t} \mid y_{1: t}, S_{1: t}, r_{t}>0\right) p\left(\alpha, \beta, \sigma \mid y_{t}^{\left(r_{t}\right)}, S_{t}^{\left(r_{t}\right)}\right) .
\end{aligned}
$$




\section{Section B: Sensitivity analyses}

18 We tested how sensitive the results are for different change point prior probabilities and for

19 different choices for the supports of the parameters that were fixed when choosing the prior

20 distributions for the parameters. We will explain the sensitivity analyses below.

\section{The change point prior}

22 We rerun the analysis on all stocks and for both the Ricker and the Beverton-Holt models.

23 We explored eight choices for the prior probabilities $1 / \lambda$, namely, the cases where the

24 hazard parameter $\lambda=\{5,10,20,100,200,1000,2000,10000\}$. These choices cover a

25 broad range of choices from change point being a very common event (on average every 5

26 years) to an extremely rare event (on average every 10000 years) a priori.

27 The medians of the posterior predictive distributions of $R_{t}$ at the end of the regimes for cod 28 using the Ricker model look identical for all the cases where $\lambda>10$ (Fig. S10). For the case

29 where $\lambda=10$, one extra regime was found, and for the case where $\lambda=5$ two extra regimes

30 were found. Since three regimes where found even with the change point prior probability of

31 one in ten thousand years, it seems there is strong evidence that at least three regimes exist

32 for cod during this time period.

33 The smoothed run length probabilities show more difference so that with a higher prior

34 probability for a change point other runs than the dominant one also obtain more probability.

35 However, the change points in 1991 and 2013 where found in all cases (Fig. S11).

36 The sequential update of the parameter posterior marginal densities shows that the smaller the

37 change point prior probability the less year to year variation in the parameter posterior 
38 distributions (for $\alpha$ see Fig. S12). However, the actual parameter posterior marginal

39 distributions at the end of the regimes differed very little.

40 Other species and tests conducted using the Beverton-Holt model showed similar tendencies, 41 and thus we have only included figures for cod using the Ricker model. The results for all 42 cases are summarized in Table S2. The table lists all the change points detected in any of the sensitivity analysis runs, and the change points detected in a particular run are marked with an ' $\mathrm{X}$ '. In summary, it seems the method is quite robust regarding the change point prior and most of the change points found in the actual analyses where also found with a lot smaller

46 prior probability.

\section{The support of the parameter priors}

48 Since, the choice for the support of the parameters was quite arbitrary, we also tested how it would affect the results if the width of the supports was doubled for some or all of the parameters. We tested all seven possibilities where the support for any of the parameters was doubled, the support for any two parameters was doubled, and the support for all three parameters was doubled. The wider supports used in the tests are reported in Table S3.

Since we had already restricted the parameter supports so that the correct parameter values seemed to be inside those supports, the only effect this test had was to give a lower value for the prior predictive density. For cod using the Ricker model the effect was that when two or more parameters had double supports, one less regime was found. Otherwise the resulting medians of the posterior predictive distributions at the end of the regimes were quite similar

58 (Fig. S13). However, as shown by the smoothed run length probabilities, the differences in 59 the run length distribution were minimal (Fig. S14), and the shifts in 1991 and 2013 were 
60 found in all of the cases. The posterior marginal densities for $\alpha$ look almost identical (Fig.

$61 \mathrm{~S} 15)$. For the density dependence parameter $\beta$ the posterior marginal densities look more

62 different when the support of $\beta$ was doubled (Fig. S16). The higher uncertainty reflects the

63 fact that the data carried only little evidence about the density dependence, and the higher

64 prior uncertainty resulted in higher posterior uncertainty throughout the time series. The

65 sensitivity analyses for other stocks and using the Beverton-Holt model yielded similar

66 results. Mostly, the sensitivity tests did not affect the number or timing of the change points,

67 but for hake using the Ricker model one more change point was found in few cases, and for

68 plaice a new change point replaced the old ones in few cases (Table S2). All in all, the

69 method was quite robust regarding the support of the parameters. 


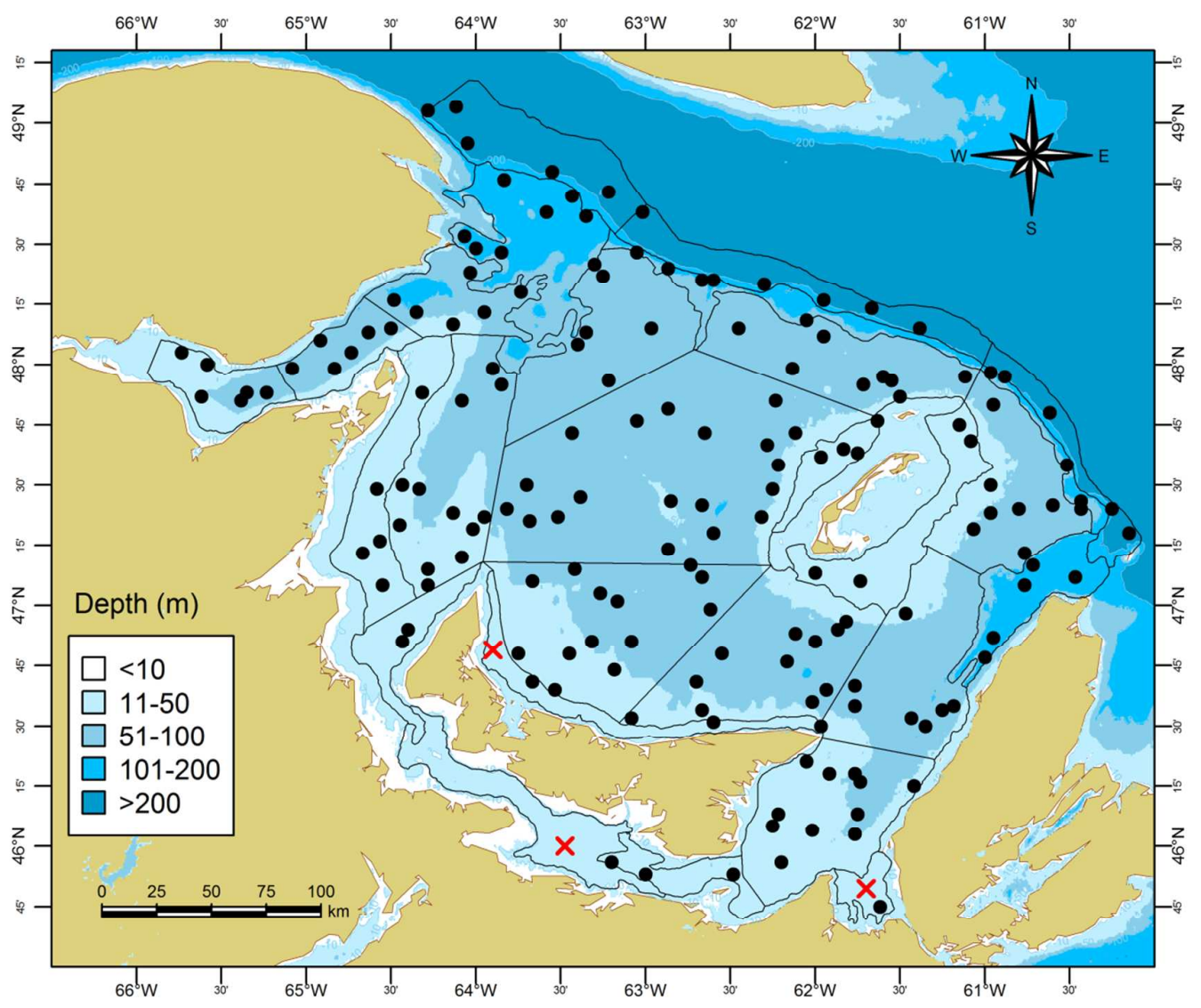

72 Figure S1. Stratification used in the September bottom-trawl survey of the southern Gulf of

73 St. Lawrence. Circles show the locations of fishing stations in the 1989 survey. " $X$ " indicates

74 three strata added in 1984 and omitted from analyses of the survey data to maintain a

75 consistent survey area. 

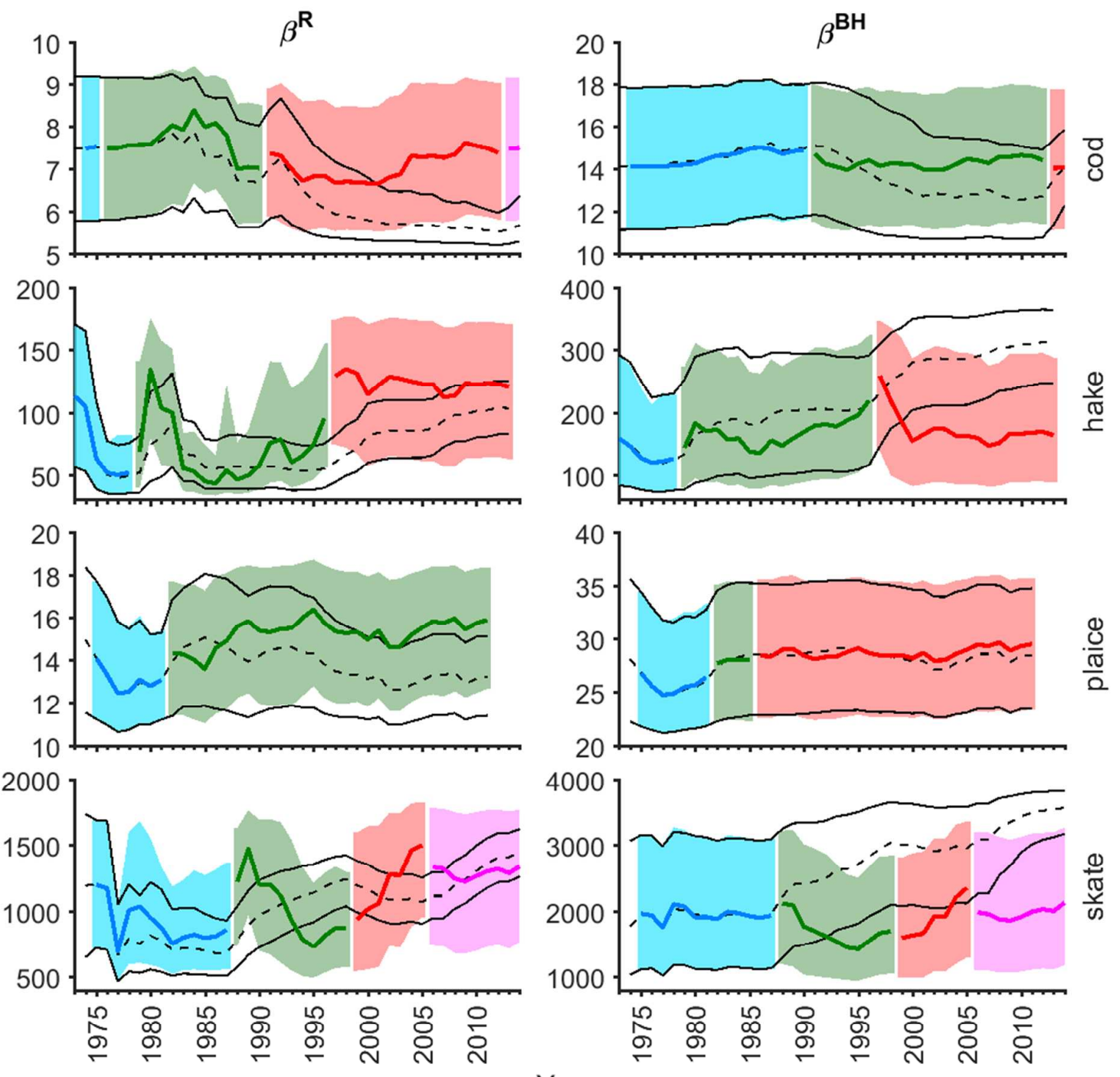

76 Figure S2. The sequential update of the posterior marginal densities of the density

77 dependence parameters $\beta^{\mathrm{R}}$ of the Ricker model and $\beta^{\mathrm{BH}}$ of the Beverton-Holt model. The

78 vertical axis has units $\frac{10^{-9}}{\mathrm{~kg}}$. Medians and $68 \%$ central probability intervals are shown.

79 Regimes are denoted with different colors. The stationary model fit is shown in black. 

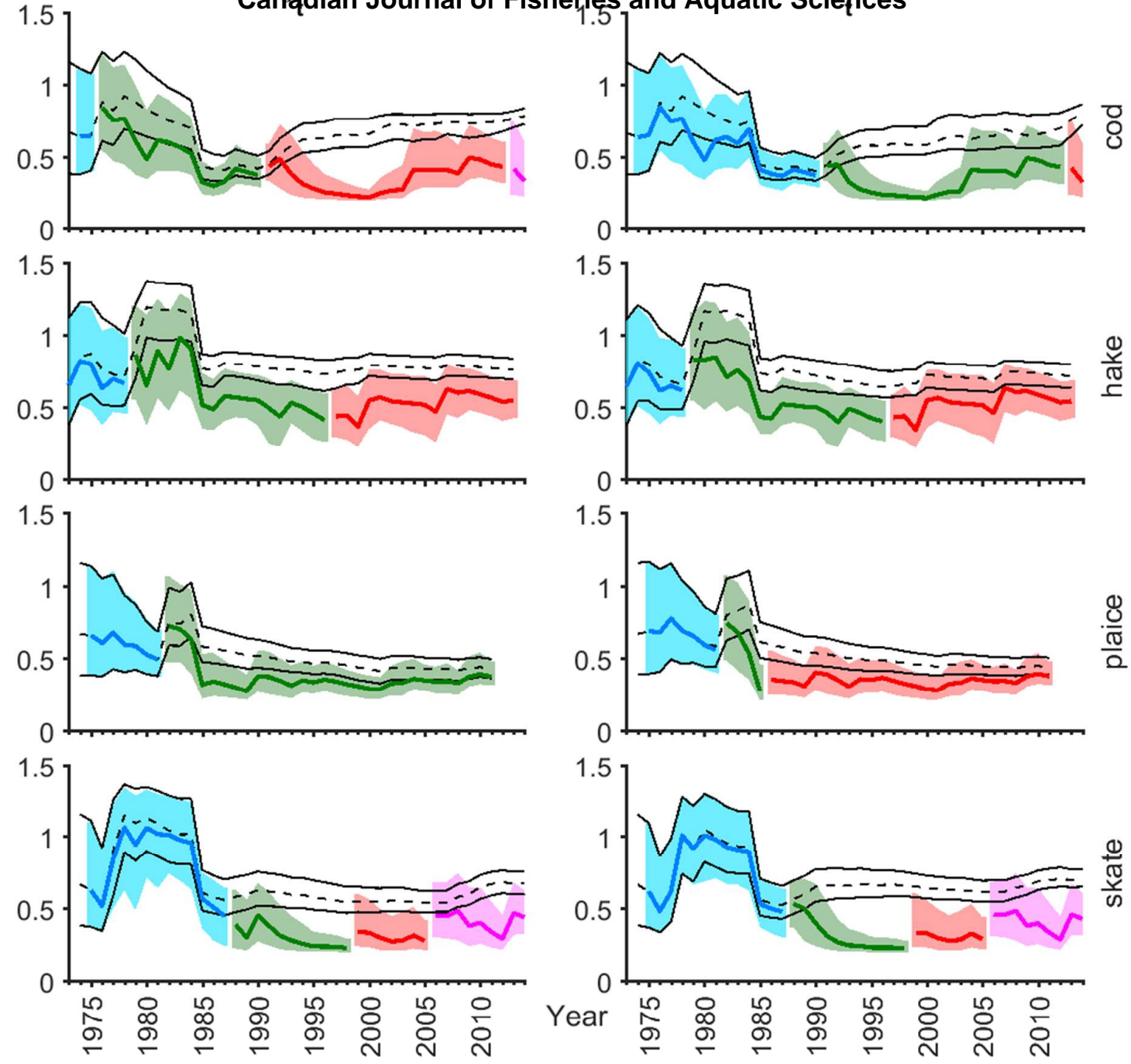

81 Figure S3. The sequential update of the posterior marginal densities of the standard deviation

82 parameters $\delta_{t}^{\mathrm{R}}$ of the Ricker model and $\delta_{t}^{\mathrm{BH}}$ of the Beverton-Holt model. Medians and

$8368 \%$ central probability intervals are shown. Regimes are denoted with different colors. The

84 constant parameter model fit is shown in black. 
Ricker
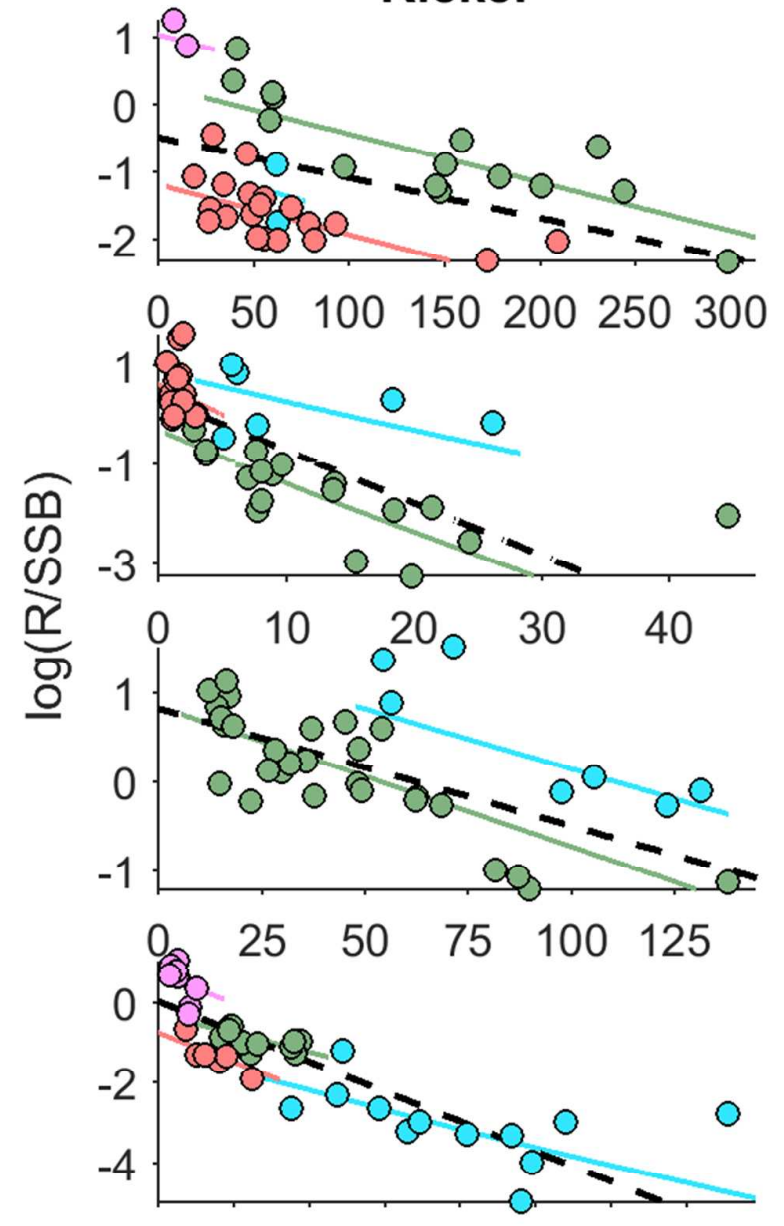

$\begin{array}{llllllll}0 & 0.5 & 1 & 1.5 & 2 & 2.5 & 3 & 3.5\end{array}$ Spawning stock biomass (thousands of metric tons)

85 Figure S4. Medians of the posterior predictive distributions of $\log \left(\frac{R}{S}\right)$ at the end of the

86 regimes for the study species. Regimes are denoted with different colors. The constant

87 parameter model fit is shown in black. 

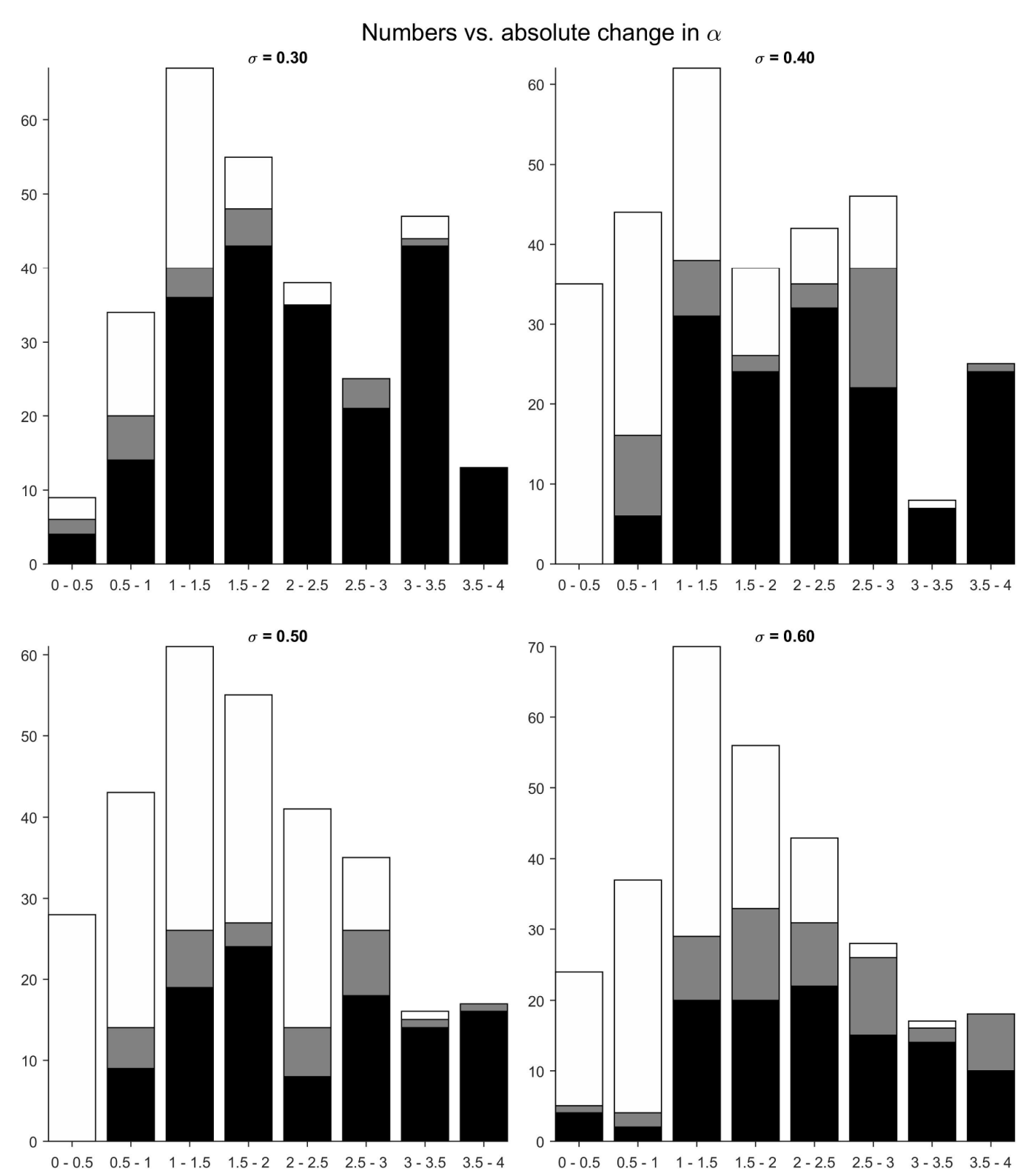

Figure S5. The results of the change point detection accuracy in the Ricker model

90 simulations for the four noise levels $(\sigma \in\{0.3,0.4,0.5,0.6\})$. The vertical axis shows the

91 number of change points and the horizontal axis shows the absolute change in $\alpha^{\mathrm{R}}$ parameter

before and after the change point. Black color denotes the change points that were inferred

93 correctly, gray color denotes those change points that were detected but with an error of \pm 1 or

$94 \pm 2$ time steps, and white denotes the rest of the change points that we considered as missed

95 by our method. 

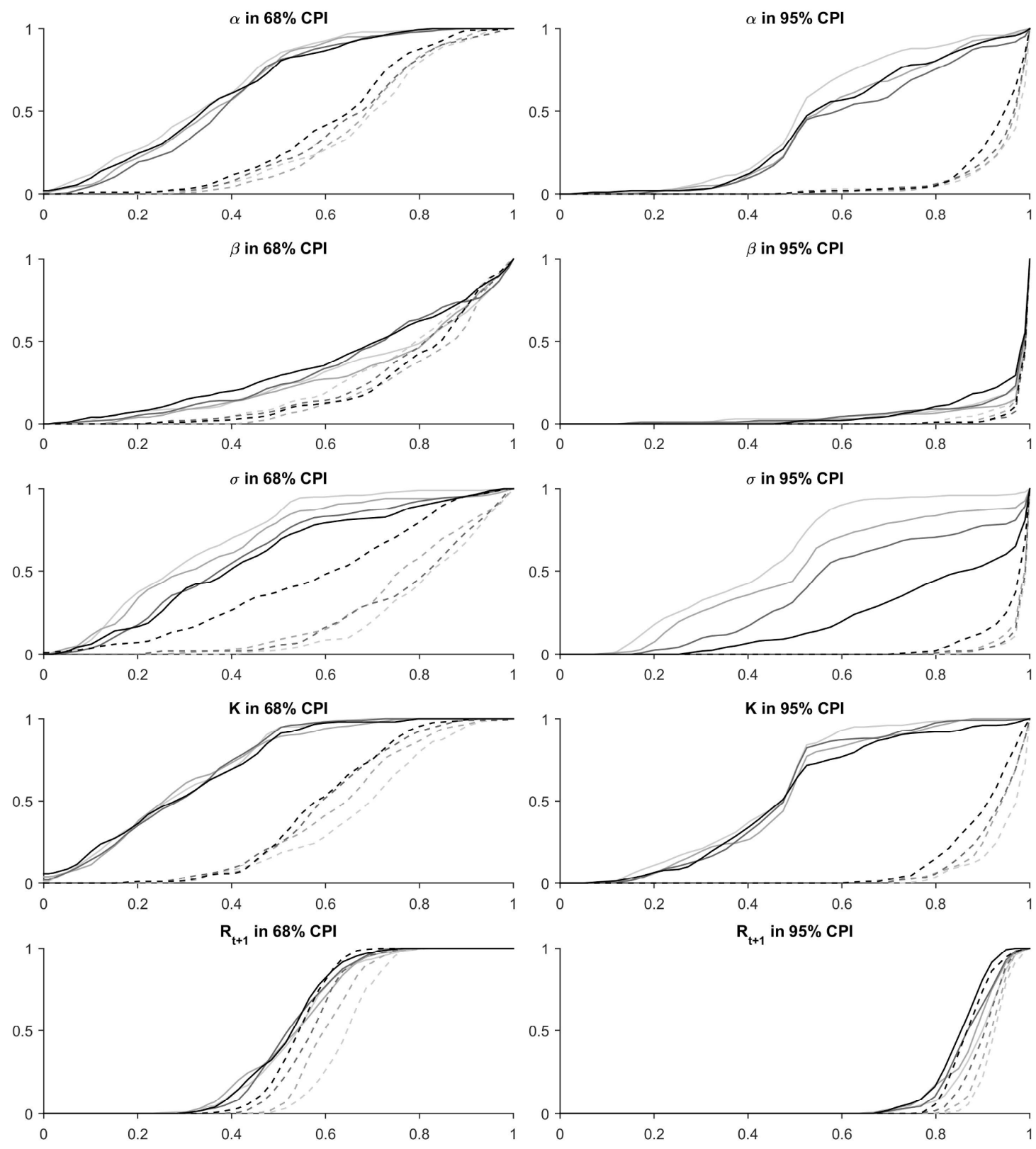

96 Figure S6. Empirical cumulative distribution functions illustrating the accuracy of the

97 parameter estimates in the Ricker model simulations. The vertical axis shows the proportion

98 of time steps within a simulation case for which the true parameter value was within the

99 posterior central probability interval (CPI), whereas the horizontal line shows the proportion

100 of all the simulation cases. The dashed line denotes the BOCPD model and the solid line 
101 denotes the model with time-invariant parameters. Different noise levels

$102(\sigma \in\{0.3,0.4,0.5,0.6\})$ are denoted with increasing darkness of the curves. The originally

103 staircase-like curves were smoothed to clarify patterns. 


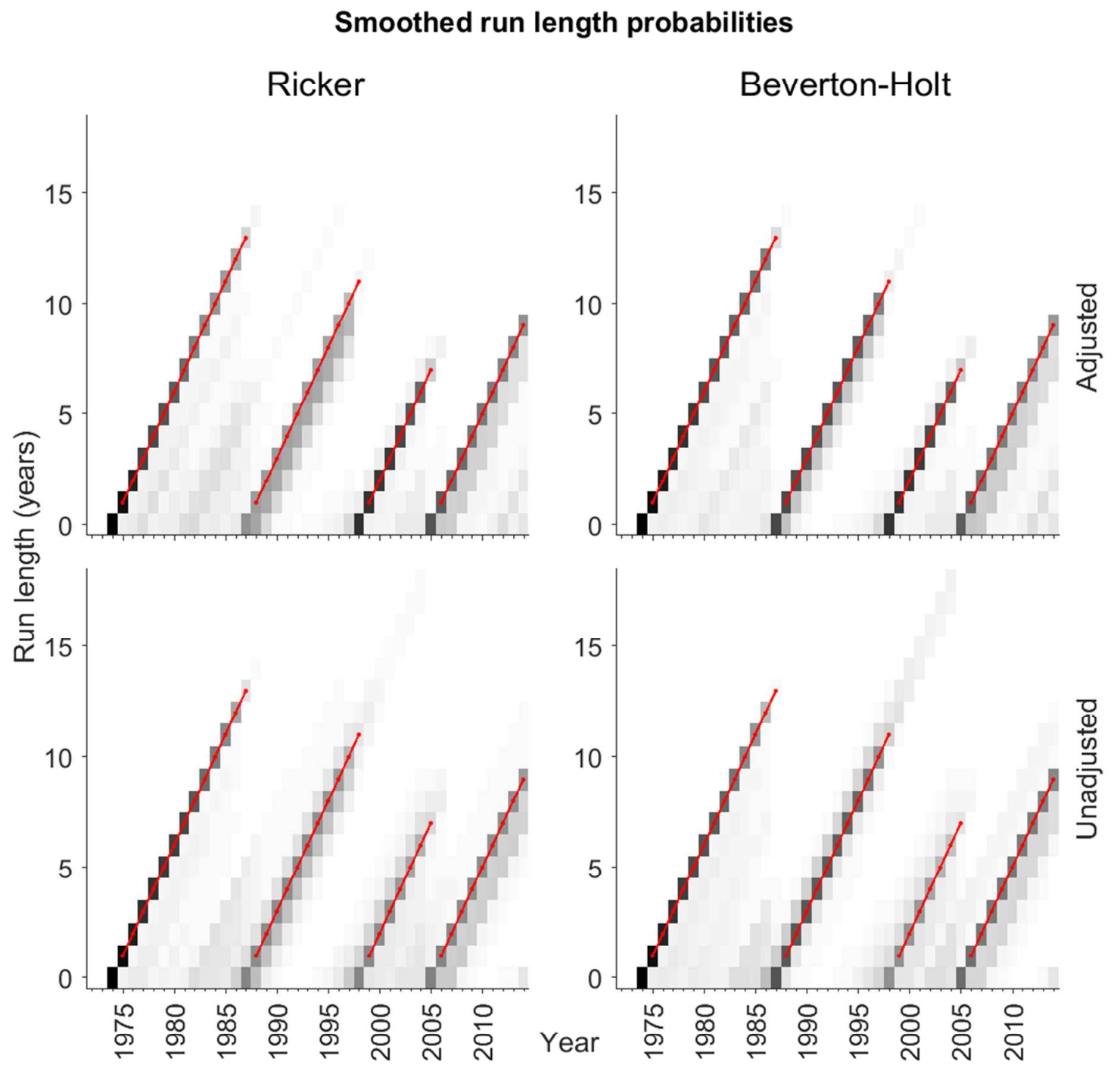

104 Figure S7. The smoothed run length probability distributions for the adjusted and unadjusted

105 data for thorny skate (Amblyraja radiata). The red lines show the most likely segmentation of 106 the data based on the smoothed run length probabilities. 


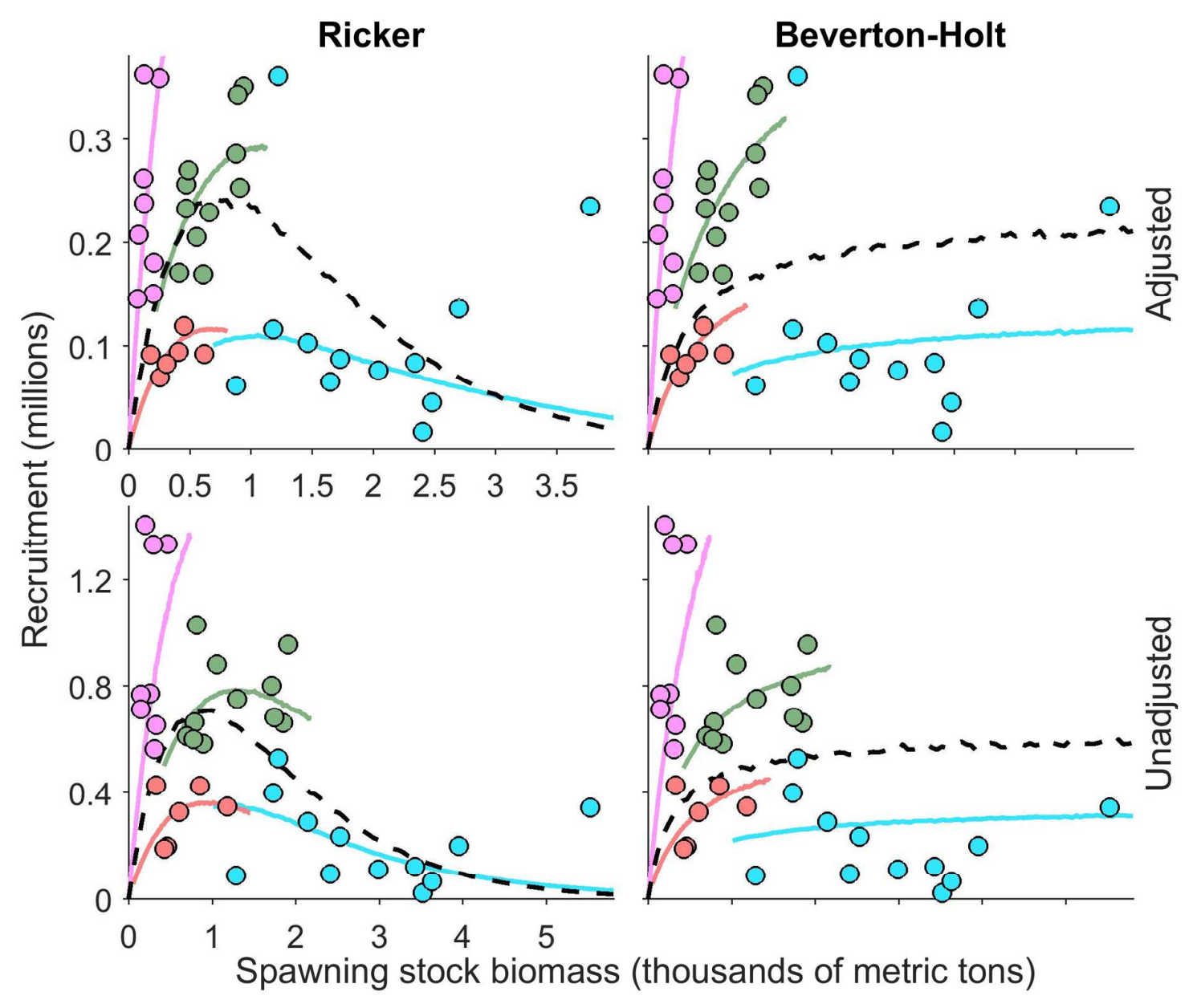

108 Figure S8. Medians of the posterior predictive distributions of $R_{t}$ for the Ricker and

109 Beverton-Holt S-R models for the adjusted and unadjusted data for thorny skate. 

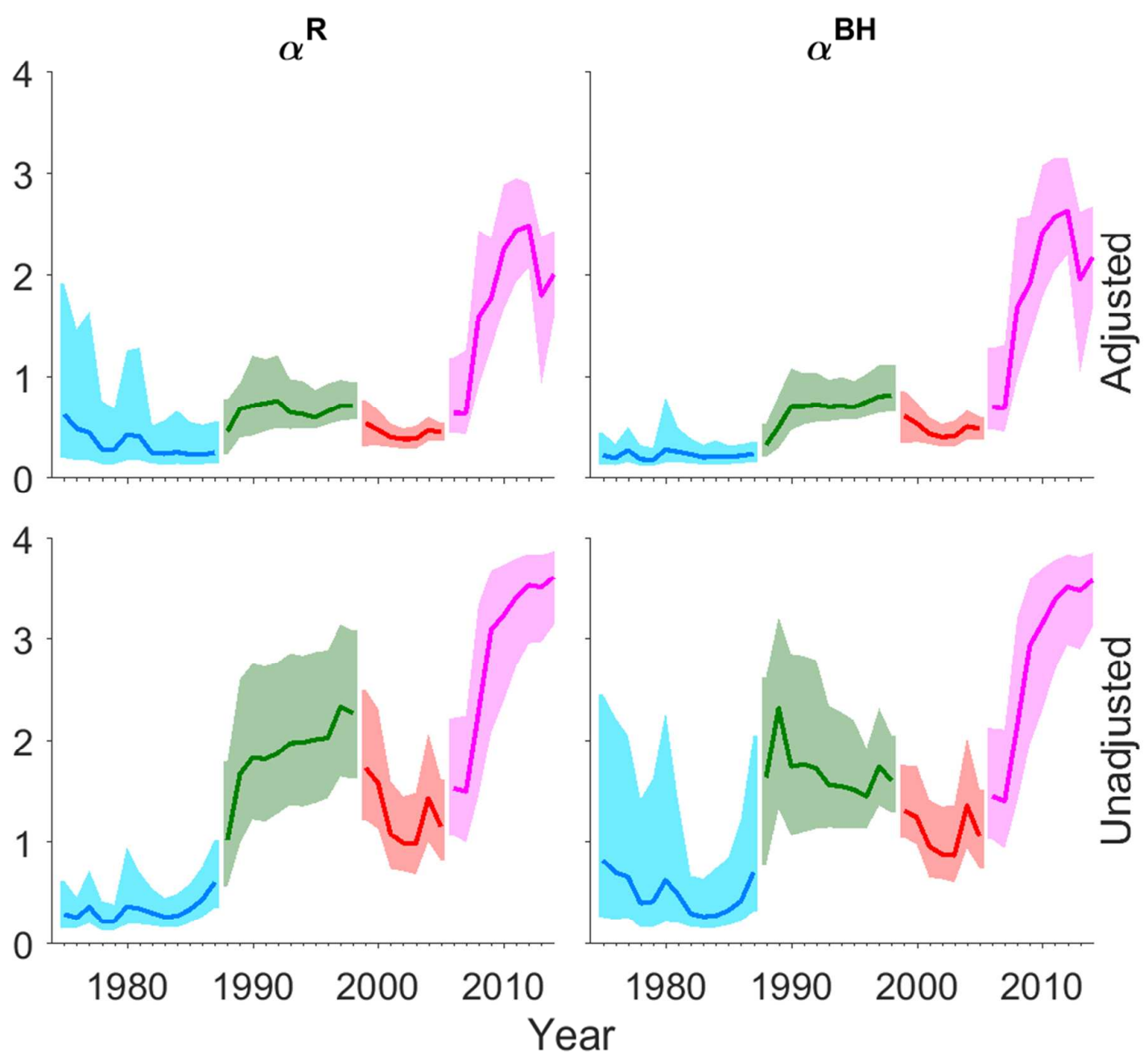

111 Figure S9. The sequential update of the posterior marginal densities of the maximum number

112 of recruits per unit of spawning stock biomass $\alpha$ for the adjusted and unadjusted data for

113 thorny skate for the study species. Output for the Ricker $\left(\alpha^{\mathrm{R}}\right)$ and Beverton-Holt $\left(\alpha^{\mathrm{BH}}\right)$

114 models are shown in left and right columns, respectively. The colored solid lines denote the

115 posterior median and the shaded areas indicate the $68 \%$ central posterior probability interval.

116 The regimes as identified using the most likely segmentation of the data are denoted by the

117 change in the color of the shading. 


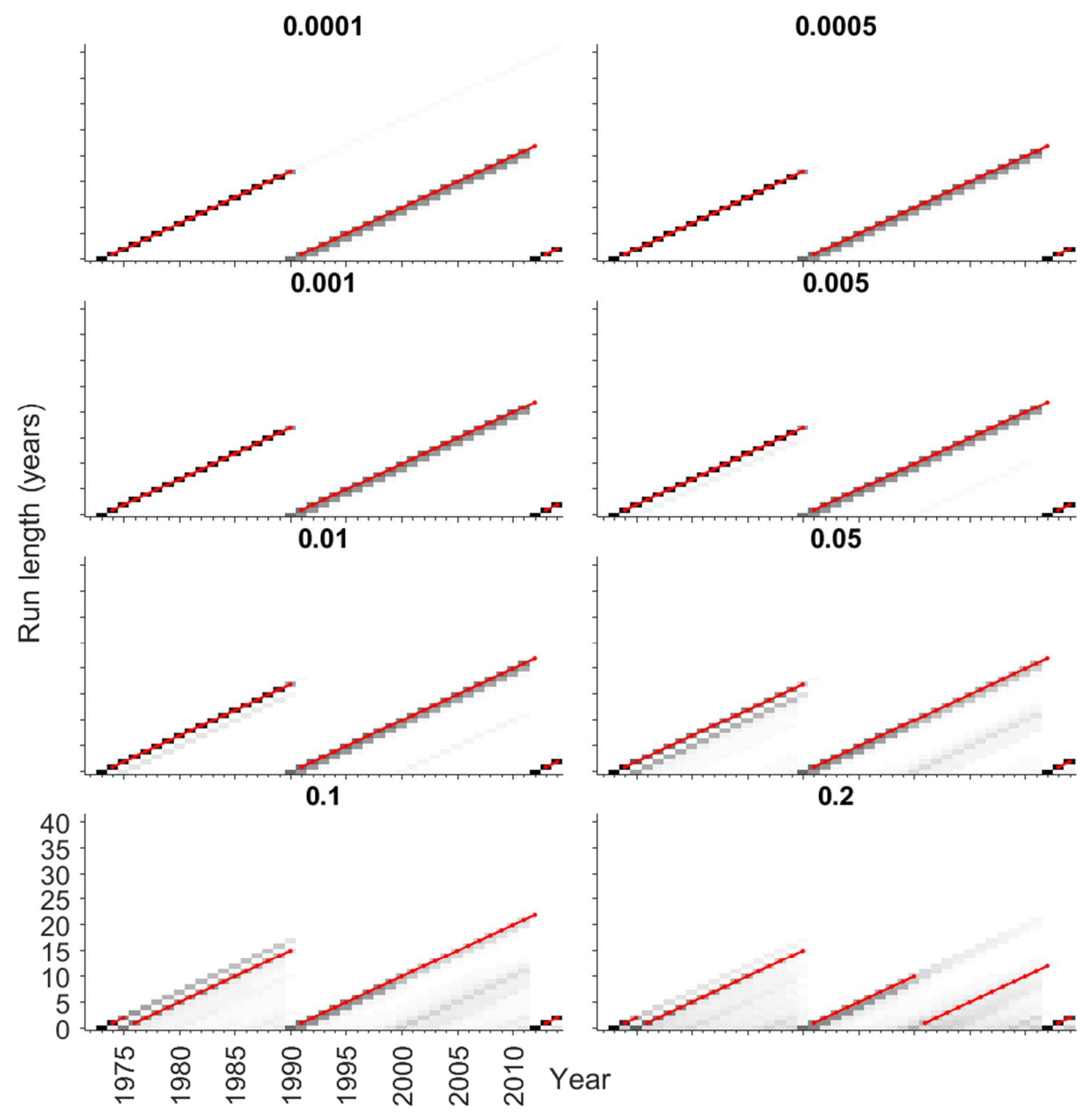

118 Figure S10. The smoothed run length probability distributions for different change point

119 prior probabilities (shown on top of the panels) for cod using the Ricker S-R model. The red

120 lines show the most likely segmentation of the data based on the smoothed run length

121 probabilities. 
0.0001

0.0005
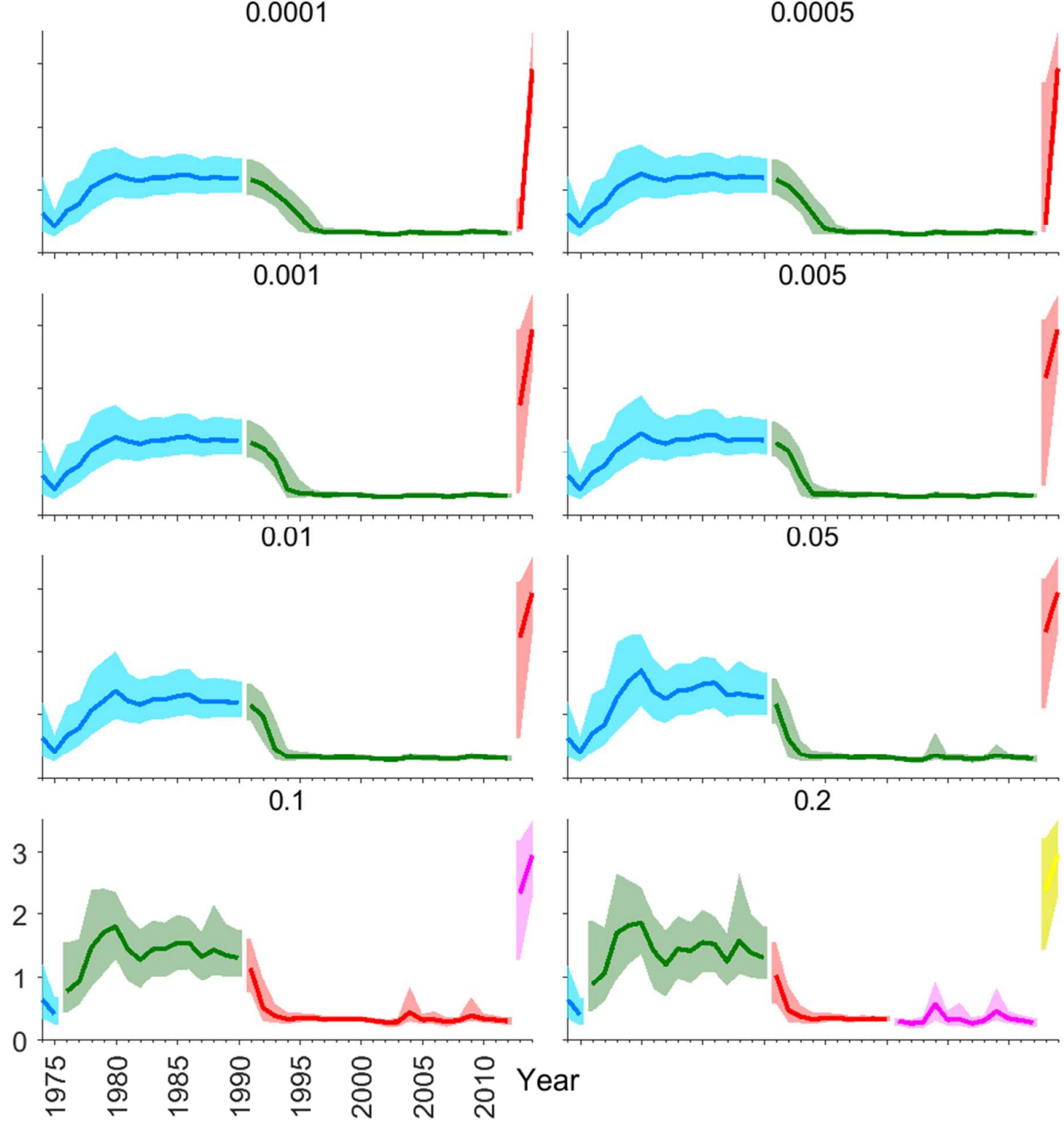

123 Figure S11. The sequential update of the posterior marginal densities of the maximum

124 number of recruits per unit of spawning stock biomass $\alpha$ for different change point prior

125 probabilities (shown on top of the panels) for cod using the Ricker S-R model. The colored

126 solid lines denote the posterior median and the shaded areas indicate the $68 \%$ central

127 posterior probability interval. The regimes as identified using the most likely segmentation of

128 the data are denoted by the change in the color of the shading. 


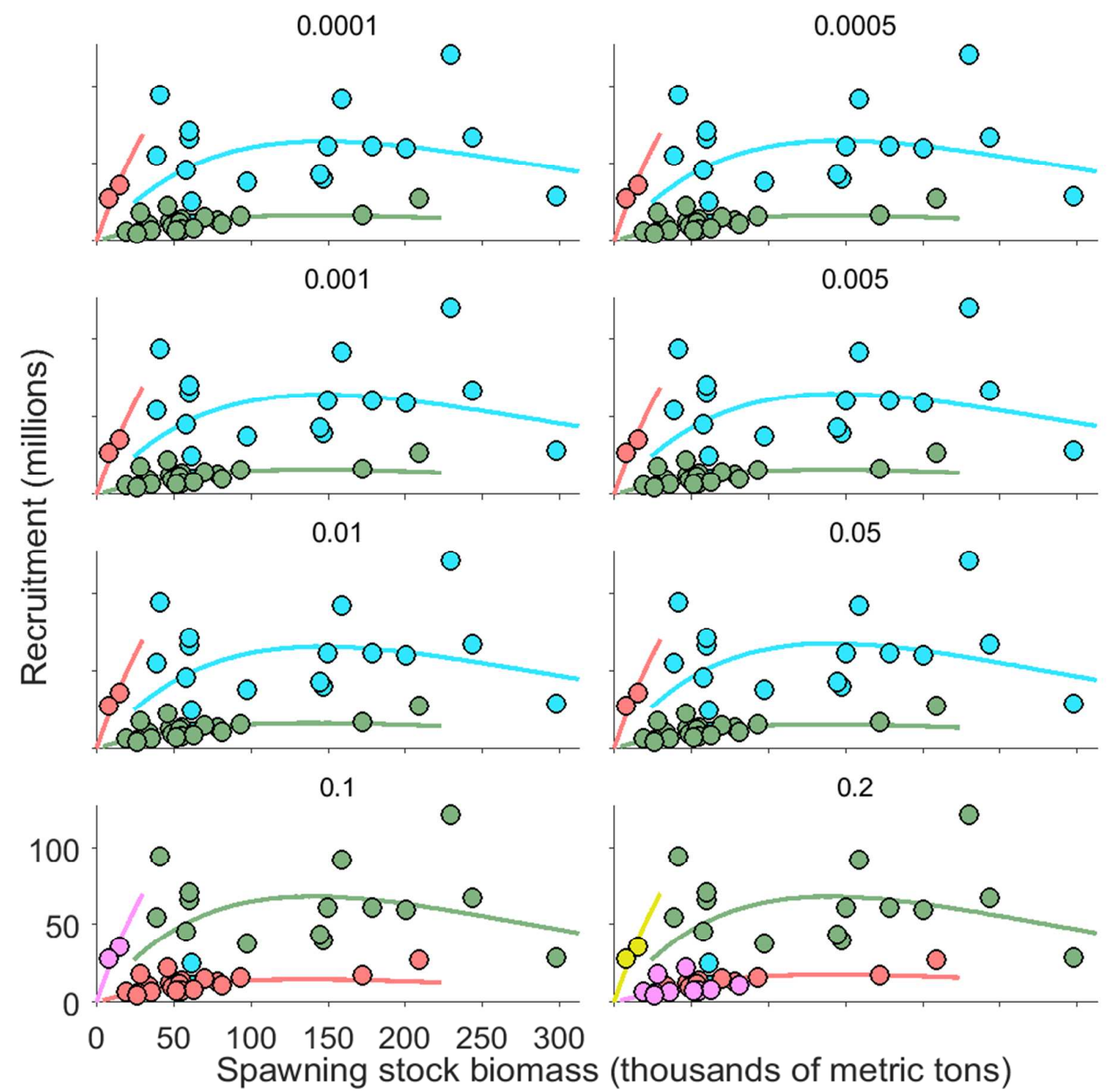

129 Figure S12. Medians of the posterior predictive distributions of $R_{t}$ at the end of the detected

130 regimes for different change point prior probabilities (shown on top of the panels) for cod

131 using the Ricker S-R model. 


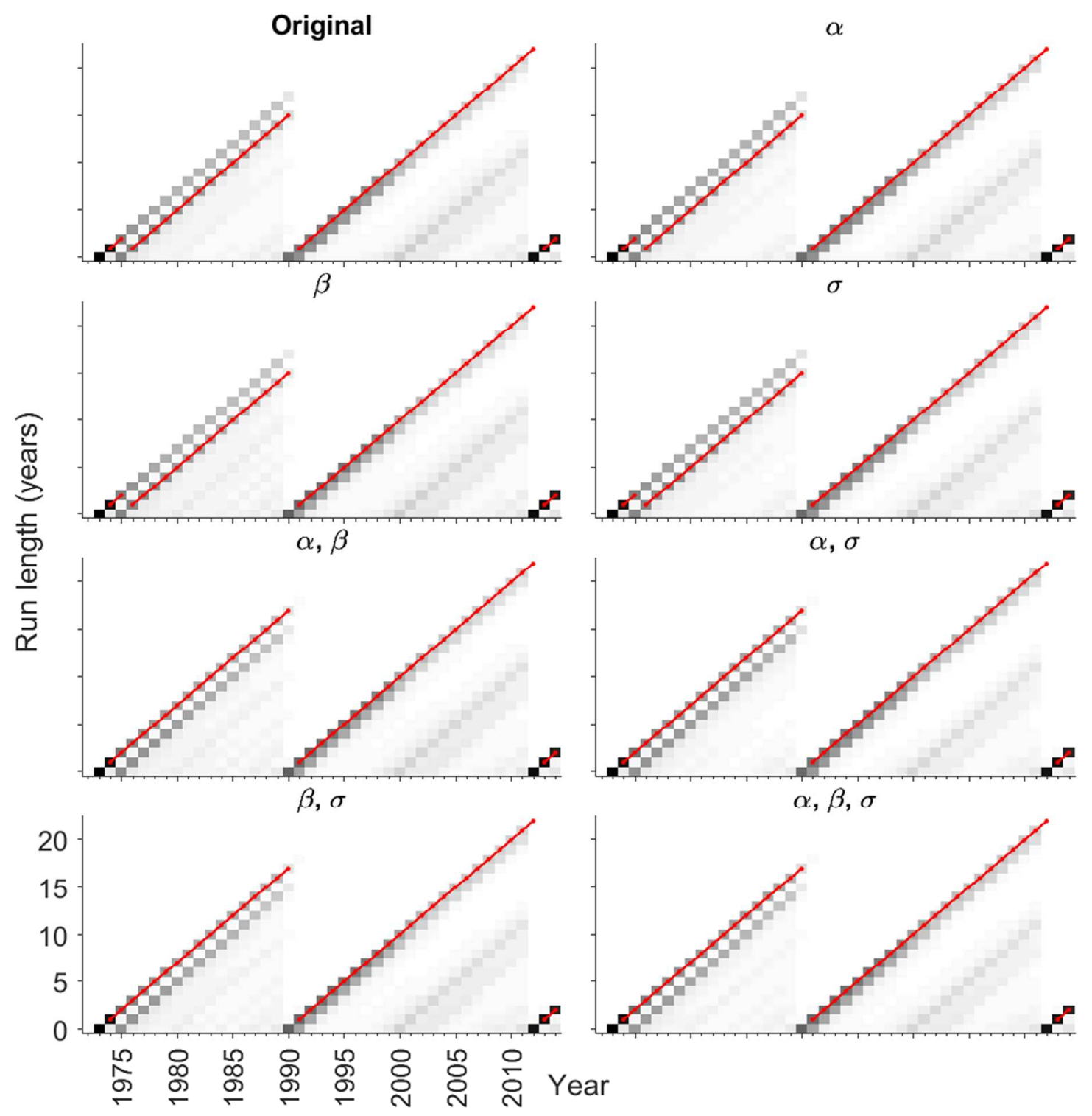

133 Figure S13. The smoothed run length probability distributions for different parameter prior

134 supports for cod using the Ricker S-R model. The symbols above the panels indicate for

135 which parameter the width of the support was doubled. The red lines show the most likely

136 segmentation of the data based on the smoothed run length probabilities. 


\section{Original}
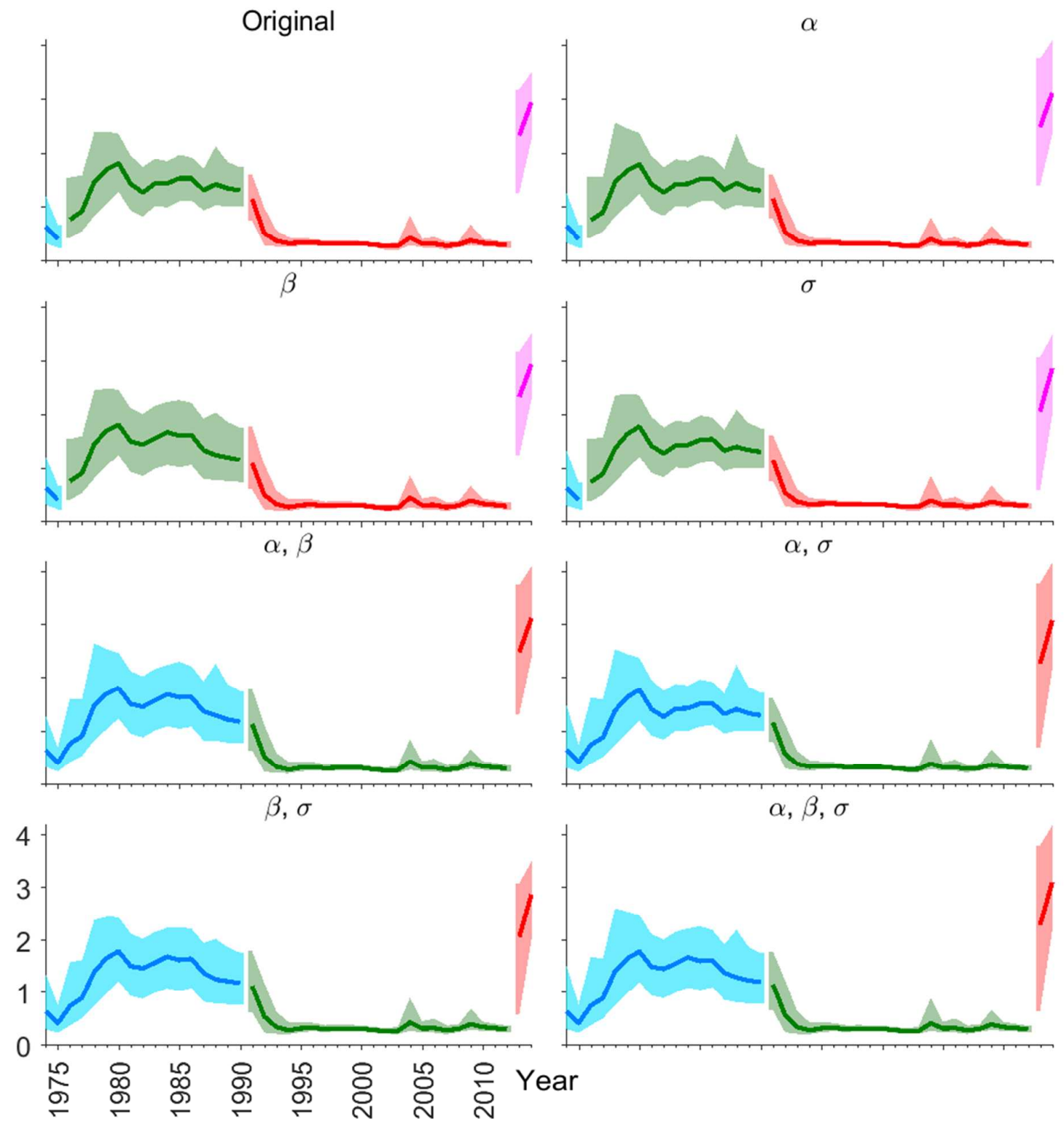

138 Figure S14. The sequential update of the posterior marginal densities of the maximum

139 number of recruits per unit of spawning stock biomass $\alpha$ for different parameter prior

140 supports for cod using the Ricker S-R model. The symbols above the panels indicate for

141 which parameter the width of the support was doubled. The colored solid lines denote the

142 posterior median and the shaded areas indicate the $68 \%$ central posterior probability interval.

143 The regimes as identified using the most likely segmentation of the data are denoted by the

144 change in the color of the shading. 

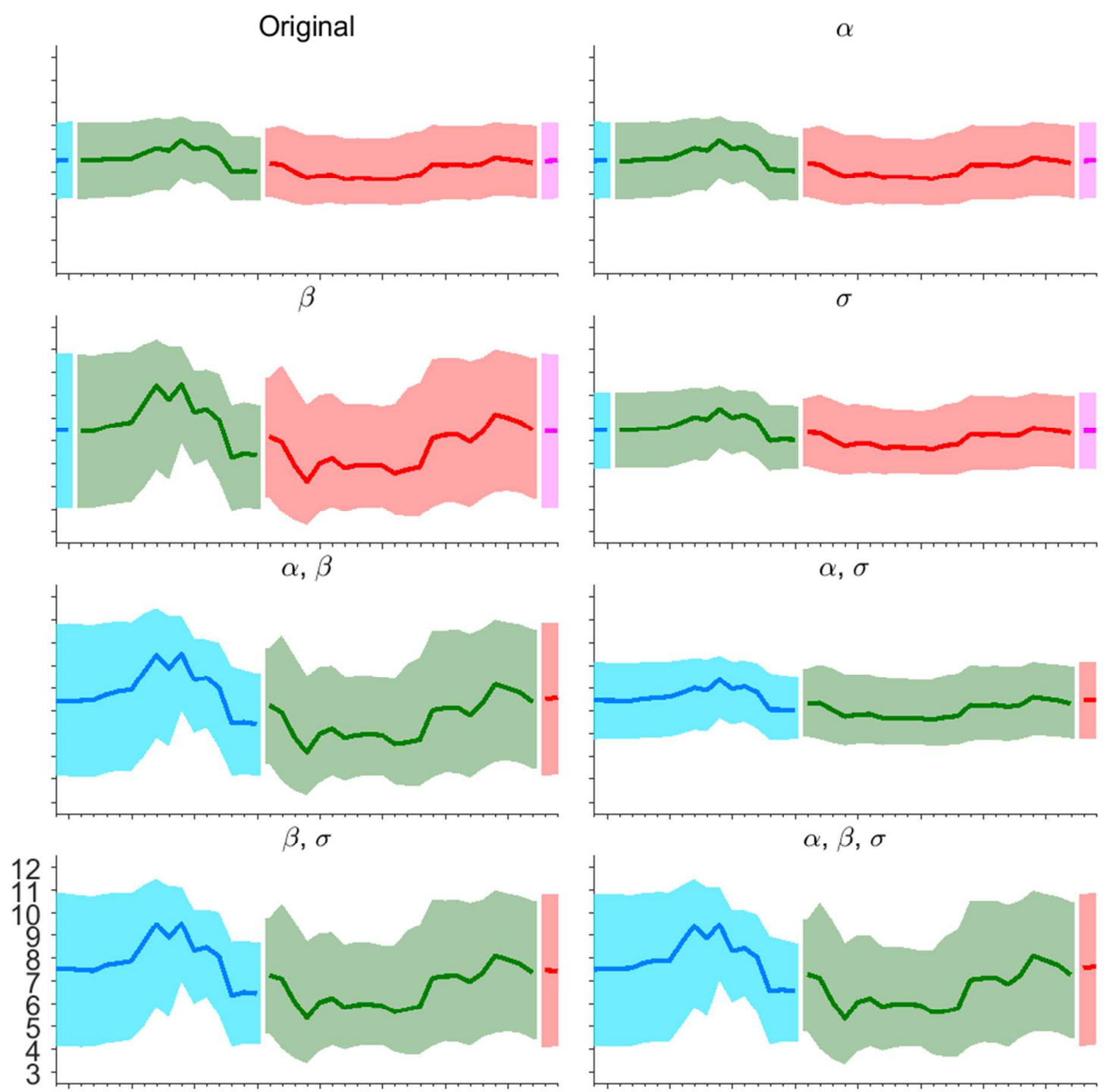

$$
\text { 잉 \& }
$$

145 Figure S15. The sequential update of the posterior marginal densities of the density

146 dependence parameter $\beta$ for different parameter prior supports for cod using the Ricker S-R

147 model. The symbols above the panels indicate for which parameter the width of the support

148 was doubled. The colored solid lines denote the posterior median and the shaded areas

149 indicate the $68 \%$ central posterior probability interval. The regimes as identified using the

150 most likely segmentation of the data are denoted by the change in the color of the shading. 

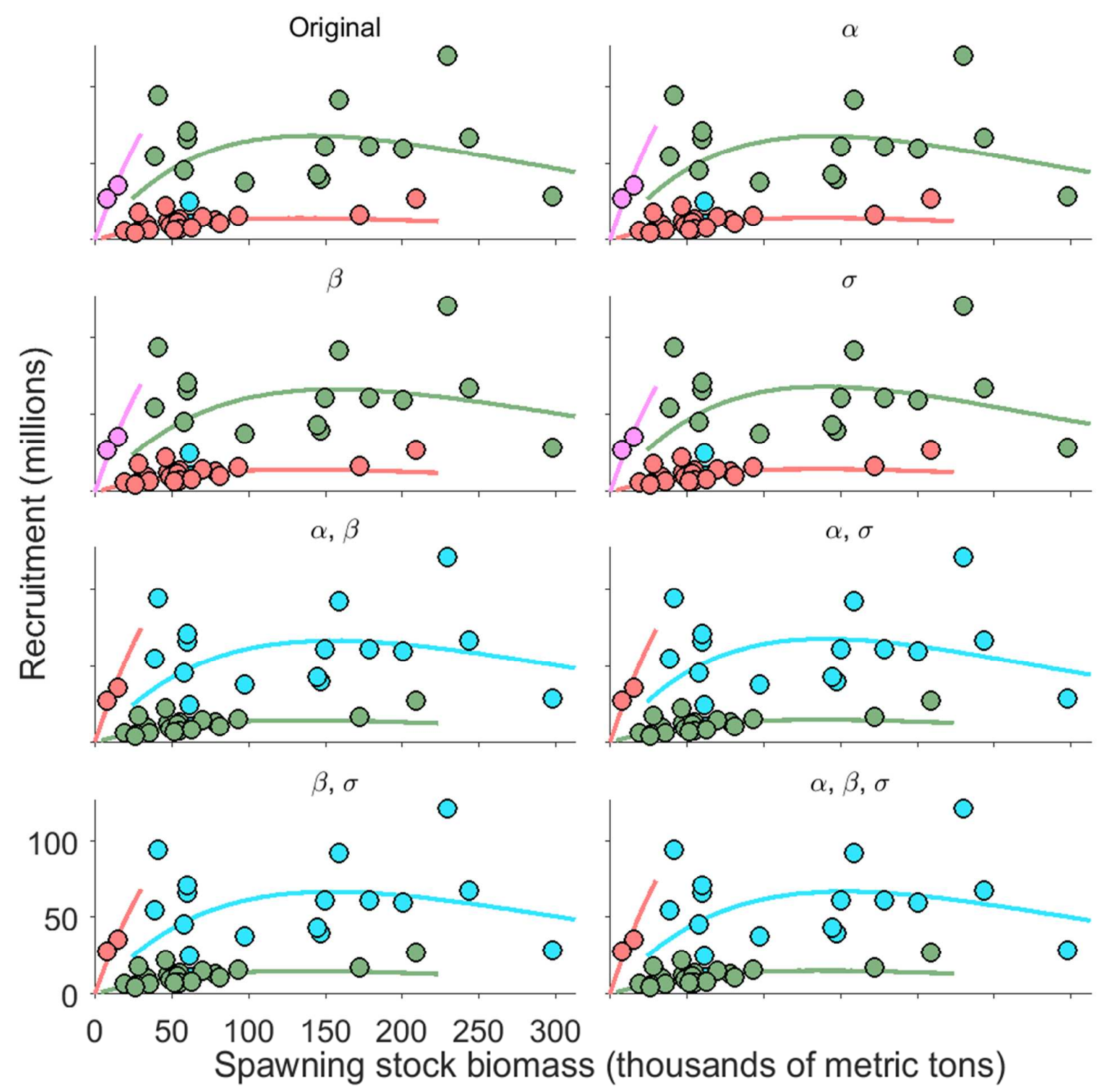

151 Figure S16. Medians of the posterior predictive distributions of $R_{t}$ for different parameter

152 prior supports for cod using the Ricker S-R model. The symbols above the panels indicate for

153 which parameter the width of the support was doubled. 


\section{Section D: Additional tables}

156 Table S1a. Catch rates of Atlantic cod and white hake in the annual bottom-trawl survey of

157 the southern Gulf of St. Lawrence. $\mathrm{R}$ is recruit abundance (fish/standard tow) and $\mathrm{S}$ is

158 spawning stock biomass ( $\mathrm{kg} /$ standard tow) in the year that the recruits were produced. Recruit

159 abundance is at age 3 years for cod and 2 years for hake. Analyses used the age-based catch

160 rates. Length-based catch rates are also included to show CVs to provide information on the

161 uncertainty in the indices for each species. (CVs are not available for the age-based indices.)

\begin{tabular}{|c|c|c|c|c|c|c|c|c|c|c|c|c|}
\hline \multirow[b]{3}{*}{ Year-Class } & \multicolumn{6}{|c|}{ Atlantic cod } & \multicolumn{6}{|c|}{ White hake } \\
\hline & \multicolumn{2}{|c|}{ Age-based } & \multicolumn{4}{|c|}{ Length-based } & \multicolumn{2}{|c|}{ Age-based } & \multicolumn{4}{|c|}{ Length-based } \\
\hline & $\mathrm{R}$ & $\mathrm{S}$ & $\mathrm{R}$ & $\mathrm{cv}$ & $\mathrm{S}$ & $\mathrm{cv}$ & $\mathrm{R}$ & $\mathrm{S}$ & $\mathrm{R}$ & $\mathrm{cv}$ & $\mathrm{S}$ & $\mathrm{cv}$ \\
\hline 1971 & 14.51 & 35.62 & 9.06 & 0.21 & 47.15 & 0.15 & 1.86 & 2.95 & 1.24 & 0.34 & 2.38 & 0.32 \\
\hline 1972 & 6.27 & 36.02 & 5.49 & 0.32 & 54.61 & 0.12 & 8.26 & 3.58 & 4.81 & 0.47 & 4.07 & 0.40 \\
\hline 1973 & 38.38 & 34.72 & 37.71 & 0.16 & 47.99 & 0.18 & 14.19 & 10.59 & 10.49 & 0.78 & 10.28 & 0.72 \\
\hline 1974 & 26.55 & 33.65 & 20.65 & 0.21 & 45.62 & 0.15 & 12.79 & 15.11 & 8.90 & 0.59 & 16.62 & 0.50 \\
\hline 1975 & 54.73 & 23.95 & 50.71 & 0.31 & 33.08 & 0.22 & 3.62 & 4.48 & 1.34 & 0.49 & 4.29 & 0.21 \\
\hline 1976 & 31.85 & 22.56 & 31.76 & 0.15 & 32.00 & 0.22 & 8.93 & 3.29 & 6.29 & 0.42 & 3.37 & 0.19 \\
\hline 1977 & 41.14 & 34.70 & 36.33 & 0.28 & 50.39 & 0.17 & 1.15 & 4.08 & 3.93 & 0.56 & 3.61 & 0.26 \\
\hline 1978 & 21.92 & 56.34 & 21.01 & 0.18 & 97.20 & 0.37 & 0.45 & 11.48 & 1.02 & 0.63 & 11.59 & 0.27 \\
\hline 1979 & 23.22 & 85.21 & 48.98 & 0.35 & 119.11 & 0.09 & 1.91 & 12.38 & 1.75 & 0.45 & 12.04 & 0.31 \\
\hline 1980 & 53.29 & 91.86 & 72.58 & 0.12 & 137.01 & 0.16 & 1.10 & 14.12 & 0.36 & 0.36 & 13.21 & 0.16 \\
\hline 1981 & 16.73 & 172.44 & 31.20 & 0.14 & 223.01 & 0.15 & 3.35 & 25.79 & 1.88 & 0.58 & 23.74 & 0.33 \\
\hline 1982 & 38.91 & 141.13 & 43.38 & 0.12 & 177.66 & 0.18 & 1.98 & 7.95 & 1.32 & 0.27 & 6.75 & 0.39 \\
\hline 1983 & 35.35 & 103.46 & 40.12 & 0.24 & 115.40 & 0.12 & 0.65 & 4.47 & 1.01 & 0.44 & 5.19 & 0.17 \\
\hline 1984 & 25.03 & 83.91 & 34.54 & 0.15 & 96.41 & 0.09 & 1.73 & 7.93 & 1.63 & 0.25 & 8.45 & 0.19 \\
\hline 1985 & 70.02 & 133.01 & 89.63 & 0.40 & 172.30 & 0.23 & 0.46 & 8.95 & 0.33 & 0.29 & 7.74 & 0.42 \\
\hline 1986 & 34.63 & 116.14 & 42.09 & 0.22 & 131.76 & 0.10 & 1.56 & 10.69 & 1.22 & 0.34 & 9.42 & 0.17 \\
\hline 1987 & 35.40 & 86.83 & 36.42 & 0.15 & 95.43 & 0.11 & 1.57 & 5.14 & 1.02 & 0.37 & 4.68 & 0.20 \\
\hline 1988 & 15.89 & 120.92 & 23.39 & 0.22 & 139.65 & 0.11 & 2.08 & 5.61 & 2.29 & 0.47 & 5.12 & 0.16 \\
\hline 1989 & 9.81 & 99.68 & 17.53 & 0.17 & 114.26 & 0.11 & 2.12 & 4.45 & 1.73 & 0.56 & 3.90 & 0.21 \\
\hline 1990 & 9.17 & 53.83 & 13.15 & 0.21 & 66.66 & 0.10 & 1.50 & 4.68 & 0.96 & 0.38 & 3.89 & 0.19 \\
\hline 1991 & 7.61 & 45.46 & 11.12 & 0.18 & 50.63 & 0.15 & 0.83 & 4.64 & 0.56 & 0.26 & 4.18 & 0.24 \\
\hline 1992 & 5.86 & 19.61 & 7.26 & 0.16 & 23.29 & 0.13 & 0.98 & 2.16 & 0.68 & 0.52 & 1.50 & 0.23 \\
\hline 1993 & 7.20 & 27.42 & 11.66 & 0.29 & 31.77 & 0.09 & 1.06 & 2.17 & 0.71 & 0.25 & 1.49 & 0.32 \\
\hline 1994 & 5.54 & 28.24 & 5.62 & 0.15 & 33.17 & 0.11 & 1.17 & 1.60 & 0.68 & 0.23 & 1.48 & 0.29 \\
\hline 1995 & 7.93 & 31.85 & 10.26 & 0.14 & 38.04 & 0.11 & 0.83 & 0.56 & 0.49 & 0.35 & 0.47 & 0.25 \\
\hline 1996 & 8.70 & 40.36 & 12.87 & 0.28 & 49.33 & 0.15 & 1.45 & 0.74 & 1.08 & 0.26 & 0.54 & 0.22 \\
\hline 1997 & 6.86 & 30.96 & 9.46 & 0.20 & 39.75 & 0.21 & 1.78 & 1.20 & 1.11 & 0.39 & 0.79 & 0.24 \\
\hline 1998 & 4.38 & 32.03 & 5.30 & 0.14 & 37.87 & 0.14 & 4.43 & 0.94 & 3.32 & 0.42 & 0.70 & 0.26 \\
\hline 1999 & 4.81 & 36.26 & 7.88 & 0.32 & 43.70 & 0.23 & 1.22 & 0.97 & 0.85 & 0.28 & 0.85 & 0.28 \\
\hline 2000 & NA & 28.82 & NA & NA & 36.07 & 0.26 & 1.71 & 1.70 & 1.17 & 0.42 & 0.69 & 0.16 \\
\hline 2001 & 12.92 & 26.81 & 17.83 & 0.33 & 34.86 & 0.28 & NA & 1.12 & NA & NA & 0.63 & 0.27 \\
\hline 2002 & 6.27 & 47.03 & 13.14 & 0.37 & 53.34 & 0.34 & 0.56 & 0.60 & 0.35 & 0.31 & 0.47 & 0.29 \\
\hline 2003 & 2.75 & NA & 4.10 & 0.25 & NA & NA & 2.51 & NA & 1.72 & 0.21 & NA & NA \\
\hline 2004 & 4.11 & 29.90 & 5.00 & 0.30 & 32.75 & 0.36 & 0.73 & 0.57 & 0.48 & 0.27 & 0.52 & 0.25 \\
\hline 2005 & 3.73 & 10.95 & 3.81 & 0.20 & 12.06 & 0.12 & 5.71 & 1.12 & 3.85 & 0.56 & 0.68 & 0.24 \\
\hline 2006 & 10.39 & 16.55 & 17.48 & 0.52 & 22.27 & 0.29 & 1.07 & 0.37 & 0.75 & 0.27 & 0.26 & 0.28 \\
\hline 2007 & 3.29 & 15.73 & 4.18 & 0.19 & 19.50 & 0.28 & 1.60 & 1.66 & 0.94 & 0.33 & 0.84 & 0.31 \\
\hline 2008 & 3.89 & 20.64 & 4.14 & 0.38 & 23.18 & 0.41 & 2.19 & 1.01 & 1.38 & 0.29 & 0.56 & 0.25 \\
\hline 2009 & 2.67 & 15.18 & 5.42 & 0.18 & 15.80 & 0.42 & 1.42 & 1.09 & 0.68 & 0.43 & 0.60 & 0.39 \\
\hline 2010 & 20.78 & 8.80 & 23.17 & 0.51 & 10.48 & 0.25 & 1.86 & 0.89 & 1.22 & 0.52 & 0.64 & 0.21 \\
\hline 2011 & 16.04 & 4.58 & 19.61 & 0.68 & 5.47 & 0.30 & 0.70 & 0.71 & 0.45 & 0.33 & 0.40 & 0.23 \\
\hline
\end{tabular}


162 Table S1b. Catch rates of American plaice and thorny skate in the annual bottom-trawl

163 survey of the southern Gulf of St. Lawrence. R is recruit abundance (fish/standard tow) and S

164 is spawning stock biomass ( $\mathrm{kg} /$ standard tow) in the year that the recruits were produced.

165 Recruit abundance is at age 4 years for plaice and 2 years post-emergence (4 years after egg

166 case deposition) for skate. Analyses used the age-based catch rates (except $\mathrm{S}$ of skate is

167 length-based). Length-based catch rates are included to show CVs, providing information on

168 the uncertainty in the indices for each species. (CVs are not available for the age-based

169 indices.)

\begin{tabular}{|c|c|c|c|c|c|c|c|c|c|c|c|c|}
\hline \multirow[b]{3}{*}{ Year-Class } & \multicolumn{6}{|c|}{ American plaice } & \multicolumn{6}{|c|}{ Thorny skate } \\
\hline & \multirow{2}{*}{\multicolumn{2}{|c|}{$\begin{array}{l}\text { Age-based } \\
\mathrm{R}\end{array}$}} & \multicolumn{4}{|c|}{ Length-based } & \multicolumn{2}{|c|}{ Age-based } & \multicolumn{4}{|c|}{ Length-based } \\
\hline & & & $\mathrm{R}$ & $\mathrm{cv}$ & $\mathrm{S}$ & $\mathrm{cv}$ & $\mathrm{R}$ & $\mathrm{S}$ & $\mathrm{R}$ & $\mathrm{cv}$ & $\mathrm{S}$ & $\mathrm{cv}$ \\
\hline 1971 & 79.41 & 32.55 & 88.20 & 0.33 & 31.29 & 0.20 & 0.049 & - & 0.091 & 0.42 & 1.354 & 0.18 \\
\hline 1972 & 123.78 & 31.48 & 177.35 & 0.23 & 36.79 & 0.26 & 0.044 & - & 0.090 & 0.57 & 1.182 & 0.27 \\
\hline 1973 & 190.24 & 41.36 & 268.20 & 0.19 & 38.28 & 0.23 & 0.136 & - & 0.275 & 0.42 & 2.183 & 0.23 \\
\hline 1974 & 64.06 & 60.92 & 102.54 & 0.17 & 61.62 & 0.14 & 0.010 & - & 0.015 & 0.82 & 1.390 & 0.17 \\
\hline 1975 & 50.52 & 56.41 & 141.58 & 0.17 & 61.49 & 0.16 & 0.038 & - & 0.055 & 0.30 & 0.954 & 0.23 \\
\hline 1976 & 68.45 & 75.92 & 109.28 & 0.24 & 97.85 & 0.11 & 0.209 & - & 0.390 & 0.82 & 0.707 & 0.25 \\
\hline 1977 & 54.32 & 71.26 & 103.64 & 0.31 & 86.21 & 0.17 & 0.079 & - & 0.160 & 0.34 & 1.563 & 0.34 \\
\hline 1978 & 15.51 & 51.90 & 28.58 & 0.39 & 67.09 & 0.31 & 0.036 & - & 0.066 & 0.66 & 0.509 & 0.26 \\
\hline 1979 & 25.95 & 79.70 & 39.32 & 0.21 & 90.59 & 0.14 & 0.026 & - & 0.049 & 0.40 & 1.434 & 0.20 \\
\hline 1980 & 17.63 & 47.25 & 22.95 & 0.17 & 68.04 & 0.20 & 0.001 & - & 0.000 & 0.00 & 0.926 & 0.21 \\
\hline 1981 & 17.39 & 50.46 & 27.88 & 0.43 & 58.56 & 0.23 & 0.051 & - & 0.104 & 0.27 & 0.999 & 0.27 \\
\hline 1982 & 30.57 & 36.15 & 33.34 & 0.25 & 45.52 & 0.21 & 0.059 & - & 0.116 & 0.25 & 0.846 & 0.36 \\
\hline 1983 & 30.45 & 39.56 & 34.33 & 0.22 & 39.69 & 0.19 & 0.067 & - & 0.128 & 0.21 & 0.684 & 0.28 \\
\hline 1984 & 26.19 & 20.68 & 32.46 & 0.17 & 25.04 & 0.09 & 0.098 & - & 0.182 & 0.24 & 0.352 & 0.23 \\
\hline 1985 & 27.31 & 27.89 & 38.16 & 0.13 & 30.39 & 0.09 & 0.119 & - & 0.222 & 0.18 & 0.321 & 0.20 \\
\hline 1986 & 56.57 & 31.24 & 70.89 & 0.15 & 36.66 & 0.14 & 0.203 & - & 0.392 & 0.25 & 0.544 & 0.17 \\
\hline 1987 & 51.17 & 26.22 & 51.05 & 0.17 & 31.72 & 0.13 & 0.146 & - & 0.284 & 0.24 & 0.526 & 0.31 \\
\hline 1988 & 40.59 & 28.04 & 48.01 & 0.24 & 35.13 & 0.19 & 0.166 & - & 0.296 & 0.30 & 0.508 & 0.15 \\
\hline 1989 & 39.14 & 21.36 & 44.27 & 0.13 & 26.91 & 0.12 & 0.099 & - & 0.195 & 0.23 & 0.238 & 0.23 \\
\hline 1990 & 25.83 & 28.49 & 41.27 & 0.16 & 38.91 & 0.11 & 0.198 & - & 0.371 & 0.42 & 0.517 & 0.14 \\
\hline 1991 & 29.24 & 35.99 & 36.96 & 0.10 & 48.28 & 0.12 & 0.133 & - & 0.241 & 0.30 & 0.381 & 0.17 \\
\hline 1992 & 18.56 & 21.83 & 32.68 & 0.12 & 28.19 & 0.09 & 0.148 & - & 0.270 & 0.21 & 0.274 & 0.26 \\
\hline 1993 & 19.48 & 17.34 & 26.67 & 0.13 & 22.05 & 0.10 & 0.156 & - & 0.295 & 0.29 & 0.283 & 0.27 \\
\hline 1994 & 22.71 & 18.45 & 32.19 & 0.14 & 24.00 & 0.11 & 0.135 & - & 0.266 & 0.35 & 0.272 & 0.28 \\
\hline 1995 & 23.29 & 16.29 & 28.85 & 0.10 & 19.11 & 0.09 & 0.055 & - & 0.094 & 0.27 & 0.236 & 0.36 \\
\hline 1996 & 17.65 & 15.42 & 27.19 & 0.12 & 19.69 & 0.09 & 0.069 & - & 0.132 & 0.32 & 0.262 & 0.25 \\
\hline 1997 & 17.46 & 9.17 & 22.88 & 0.10 & 12.94 & 0.08 & 0.040 & - & 0.074 & 0.28 & 0.149 & 0.20 \\
\hline 1998 & 10.47 & 12.97 & 18.86 & 0.11 & 15.13 & 0.09 & 0.053 & - & 0.091 & 0.33 & 0.359 & 0.24 \\
\hline 1999 & NA & 9.86 & NA & NA & 12.53 & 0.09 & NA & - & NA & NA & 0.191 & 0.24 \\
\hline 2000 & 25.71 & 9.86 & 29.18 & 0.13 & 12.32 & 0.12 & 0.053 & - & 0.094 & 0.40 & 0.105 & 0.29 \\
\hline 2001 & 19.07 & 8.20 & 33.60 & 0.18 & 11.89 & 0.13 & 0.048 & - & 0.085 & 0.39 & 0.179 & 0.26 \\
\hline 2002 & 19.85 & 7.13 & 30.54 & 0.09 & 9.41 & 0.07 & 0.104 & - & 0.201 & 0.25 & 0.121 & 0.37 \\
\hline 2003 & 14.19 & NA & 26.23 & 0.12 & NA & NA & 0.072 & - & 0.126 & 0.22 & NA & NA \\
\hline 2004 & 17.94 & 8.76 & 27.28 & 0.10 & 8.93 & 0.10 & 0.138 & - & 0.262 & 0.29 & 0.073 & 0.30 \\
\hline 2005 & 8.37 & 8.53 & 20.23 & 0.10 & 11.07 & 0.09 & 0.208 & - & 0.398 & 0.35 & 0.147 & 0.23 \\
\hline 2006 & 29.18 & 9.43 & 49.09 & 0.10 & 10.19 & 0.09 & 0.210 & - & 0.395 & 0.34 & 0.074 & 0.38 \\
\hline 2007 & 19.57 & 10.46 & 35.28 & 0.11 & 10.56 & 0.08 & 0.120 & . & 0.232 & 0.34 & 0.048 & 0.46 \\
\hline 2008 & & & & & & & 0.152 & & 0.270 & 0.50 & 0.070 & 0.50 \\
\hline 2009 & & & & & & & 0.087 & - & 0.164 & 0.31 & 0.117 & 0.34 \\
\hline 2010 & & & & & & & 0.084 & - & 0.159 & 0.48 & 0.043 & 0.54 \\
\hline
\end{tabular}


170 Table S2. Summary of the sensitivity analyses. We have listed all years when a change point

171 was found in any of the sensitivity test runs (columns) and marked whether the particular

172 change point was found in a given sensitivity run (rows). The bolded rows show the

173 parameterization used for the actual analyses. The third column from the left shows either the

174 change point prior probability used or the symbol of the parameter whose support was

175 doubled.

\begin{tabular}{|c|c|c|c|c|c|c|c|c|c|c|c|c|c|c|c|c|c|c|c|c|c|c|c|}
\hline & \multicolumn{4}{|c|}{$\operatorname{cod}$} & \multicolumn{5}{|c|}{ hake } & \multicolumn{6}{|c|}{ plaice } & \multicolumn{6}{|c|}{ skate } \\
\hline & & & 1976 & 1991 & 2001 & 2013 & 1979 & 1988 & 1993 & 1997 & 2013 & 1978 & 1982 & 1986 & 1994 & 2004 & 2010 & 1983 & 1988 & 1999 & 2006 & 2007 & 2013 \\
\hline \multirow{16}{*}{ 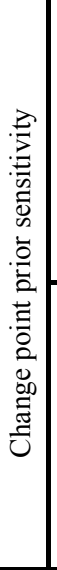 } & \multirow{8}{*}{ 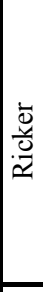 } & 0.0001 & & $\mathrm{X}$ & & $\mathrm{X}$ & & & & & & & & & & & & & & & & & \\
\hline & & 0.0005 & & $\mathrm{X}$ & & $X$ & & & & & & & & & & & & & & & & $X$ & \\
\hline & & 0.001 & & $X$ & & $X$ & & & & & & & & & & & & & & & & $\mathrm{X}$ & \\
\hline & & 0.005 & & $\mathrm{X}$ & & $\mathrm{X}$ & & & & $\mathrm{X}$ & & & $X$ & & & & & & & & $\mathrm{X}$ & & \\
\hline & & 0.01 & & $\mathrm{X}$ & & $X$ & $X$ & & & $\mathrm{X}$ & & & $X$ & & & & & & & & $X$ & & \\
\hline & & 0.05 & & $\mathrm{X}$ & & $\mathrm{X}$ & $\mathrm{X}$ & & & $\mathrm{X}$ & & & $X$ & & & & & & $X$ & $X$ & $\mathrm{X}$ & & \\
\hline & & 0.1 & $\mathbf{X}$ & $\mathbf{X}$ & & $\mathbf{X}$ & $\mathbf{X}$ & & & $\mathbf{X}$ & & & $\mathbf{X}$ & & & & & & $\mathbf{X}$ & $\mathbf{X}$ & $\mathbf{X}$ & & \\
\hline & & 0.2 & $\mathrm{X}$ & $\mathrm{X}$ & $\mathrm{X}$ & $\mathrm{X}$ & $\mathrm{X}$ & $\mathrm{X}$ & & $\mathrm{X}$ & $\mathrm{X}$ & & $X$ & & $\mathrm{X}$ & $\mathrm{X}$ & $\mathrm{X}$ & $\mathrm{X}$ & $X$ & $\mathrm{X}$ & $\mathrm{X}$ & & \\
\hline & \multirow{8}{*}{ 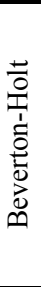 } & 0.0001 & & $\mathrm{X}$ & & $\mathrm{X}$ & & & & & & & & & & & & & & & $\mathrm{X}$ & & \\
\hline & & 0.0005 & & $\mathrm{X}$ & & $\mathrm{X}$ & & & & 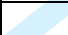 & 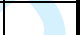 & & & & & & & & $\mathrm{X}$ & & $\mathrm{X}$ & & \\
\hline & & 0.001 & & $\mathrm{X}$ & & $X$ & & & & 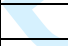 & 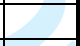 & & & & & & & & $X$ & & $\mathrm{X}$ & & \\
\hline & & 0.005 & & $\mathrm{X}$ & & $\mathrm{X}$ & $\mathrm{X}$ & & & $\mathrm{X}$ & 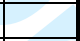 & & & & & & & & $\mathrm{X}$ & $\mathrm{X}$ & $\mathrm{X}$ & & \\
\hline & & 0.01 & & $\mathrm{X}$ & & $X$ & $\mathrm{X}$ & & & $\mathrm{X}$ & 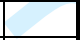 & 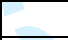 & & & & & & & $\mathrm{X}$ & $X$ & $\mathrm{X}$ & & \\
\hline & & 0.05 & & $X$ & & $X$ & $\mathrm{X}$ & & & $X$ & & $\mathbf{F}$ & $X$ & $X$ & & & & & $\mathrm{X}$ & $X$ & $X$ & & \\
\hline & & 0.1 & & $\mathbf{X}$ & & $\mathbf{X}$ & $\mathbf{X}$ & & & $\mathbf{X}$ & 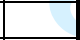 & $\mathrm{Z}$ & $\mathbf{X}$ & $\mathbf{X}$ & & & & & $\mathbf{X}$ & $\mathbf{X}$ & $\mathbf{X}$ & & \\
\hline & & 0.2 & $\mathrm{X}$ & $\mathrm{X}$ & $\mathrm{X}$ & $\mathrm{X}$ & $\mathrm{X}$ & $\mathrm{X}$ & & $\mathrm{X}$ & $\mathrm{X}$ & 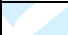 & $X$ & $\mathrm{X}$ & $\mathrm{X}$ & $\mathrm{X}$ & & & $\mathrm{X}$ & $\mathrm{X}$ & $\mathrm{X}$ & & $\mathrm{X}$ \\
\hline \multirow{16}{*}{ 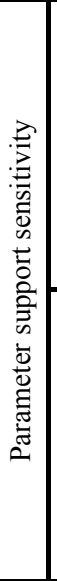 } & \multirow{8}{*}{$\frac{\vec{d}}{\frac{\vec{v}}{\vec{c}}}$} & Orig. & $\bar{X}$ & $\mathrm{X}$ & & $\bar{X}$ & $\bar{X}$ & & & $\mathrm{X}$ & & & $\bar{X}$ & & & & & & $\bar{X}$ & $\bar{X}$ & $\bar{X}$ & & \\
\hline & & $\alpha$ & $\mathrm{X}$ & $\mathrm{X}$ & & $\mathrm{X}$ & $\mathrm{X}$ & & & $\mathrm{X}$ & & & $\mathrm{X}$ & & & & & & $\mathrm{X}$ & $\mathrm{X}$ & $\mathrm{X}$ & & \\
\hline & & $\beta$ & $\mathrm{X}$ & $\mathrm{X}$ & & $\mathrm{X}$ & $\mathrm{X}$ & & $\mathrm{X}$ & $\mathrm{X}$ & & & $X$ & & & & & & $\mathrm{X}$ & $\mathrm{X}$ & $\mathrm{X}$ & & \\
\hline & & $\sigma$ & $X$ & $\mathrm{X}$ & & $X$ & $\mathrm{X}$ & & & $X$ & & & $X$ & & & & & & $\mathrm{X}$ & $X$ & $X$ & & \\
\hline & & $\alpha, \beta$ & & $\mathrm{X}$ & & $\mathrm{X}$ & $X$ & & $\mathrm{X}$ & $\mathrm{X}$ & & & $X$ & & & & & & $X$ & $\mathrm{X}$ & $\mathrm{X}$ & & \\
\hline & & $\alpha, \sigma$ & & $\mathrm{X}$ & & $X$ & $X$ & & & $X$ & & & $X$ & & & & & & $X$ & $X$ & $\mathrm{X}$ & & \\
\hline & & $\beta, \sigma$ & & $\mathrm{X}$ & & $X$ & $\mathrm{X}$ & & $X$ & $\mathrm{X}$ & & & $X$ & & & & & & $X$ & $\mathrm{X}$ & $X$ & & \\
\hline & & $\alpha, \beta, \sigma$ & & $\mathrm{X}$ & & $X$ & $\mathrm{X}$ & & $\mathrm{X}$ & $\mathrm{X}$ & & & $\mathrm{X}$ & & & & & & $X$ & $\mathrm{X}$ & $\mathrm{X}$ & & \\
\hline & \multirow{8}{*}{ 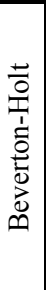 } & Orig. & & $\mathrm{X}$ & & $\bar{X}$ & $\mathbf{X}$ & & & $\mathbf{X}$ & & & $\bar{X}$ & $\bar{X}$ & & & & & $\bar{X}$ & $\mathbf{X}$ & $\bar{X}$ & & \\
\hline & & $\alpha$ & & $X$ & & $X$ & $X$ & & & $\mathrm{X}$ & & $\mathrm{X}$ & & & & & & & $\bar{X}$ & $\mathrm{X}$ & $\bar{X}$ & & \\
\hline & & $\beta$ & & $\mathrm{X}$ & & $\mathrm{X}$ & $\mathrm{X}$ & & & $\mathrm{X}$ & & & $X$ & $X$ & & & & & $X$ & $\mathrm{X}$ & $\mathrm{X}$ & & \\
\hline & & $\sigma$ & & $\mathrm{X}$ & & $\mathrm{X}$ & $\mathrm{X}$ & & & $\mathrm{X}$ & & & $\mathrm{X}$ & $X$ & & & & & $\mathrm{X}$ & $\mathrm{X}$ & $\mathrm{X}$ & & \\
\hline & & $\alpha, \beta$ & & $\mathrm{X}$ & & $\mathrm{X}$ & $X$ & & & $X$ & & $X$ & & & & & & & $X$ & $\mathrm{X}$ & $\mathrm{X}$ & & \\
\hline & & $\alpha, \sigma$ & & $X$ & & $X$ & $\mathrm{X}$ & & & $X$ & & $X$ & & & & & & & $X$ & $\mathrm{X}$ & $X$ & & \\
\hline & & $\beta, \sigma$ & & $\mathrm{X}$ & & $X$ & $\mathrm{X}$ & & & $X$ & & & $\mathbf{X}$ & $\mathbf{X}$ & & & & & $X$ & $X$ & $X$ & & \\
\hline & & $\alpha, \beta, \sigma$ & & $\mathrm{X}$ & & $\mathrm{X}$ & $\mathrm{X}$ & & & $\mathrm{X}$ & & $\mathrm{X}$ & & & & & & & $\mathrm{X}$ & $\mathrm{X}$ & $\mathrm{X}$ & & \\
\hline
\end{tabular}

176

177 
178 Table S3. The wider parameter supports used in the sensitivity analyses.

179

\begin{tabular}{|l|c|c|c|c|c|c|}
\cline { 2 - 7 } \multicolumn{1}{c|}{} & \multicolumn{3}{c|}{ Ricker } & \multicolumn{3}{c|}{ Beverton-Holt } \\
\cline { 2 - 7 } \multicolumn{1}{c|}{$\alpha$} & $\beta$ & $\sigma$ & $\alpha$ & $\beta$ & $\sigma$ \\
\hline cod & {$[0.3,2.7]$} & {$\left[2.5 \cdot 10^{-9}, 12.5 \cdot 10^{-9}\right]$} & {$[0.3,2.7]$} & {$[0.1,7.9]$} & {$\left[5 \cdot 10^{-9}, 25 \cdot 10^{-9}\right]$} & {$[0.3,2.7]$} \\
\hline hake & {$[0.1,11.9]$} & {$\left[10 \cdot 10^{-9}, 350 \cdot 10^{-9}\right]$} & {$[0.3,2.7]$} & {$[0.1,11.9]$} & {$\left[10 \cdot 10^{-9}, 690 \cdot 10^{-9}\right]$} & {$[0.3,2.7]$} \\
\hline plaice & {$[0.1,11.7]$} & {$\left[5 \cdot 10^{-9}, 25 \cdot 10^{-9}\right]$} & {$[0.3,2.7]$} & {$[0.1,11.7]$} & {$\left[10 \cdot 10^{-9}, 50 \cdot 10^{-9}\right]$} & {$[0.3,2.7]$} \\
\hline skate & {$[0.1,7.9]$} & {$\left[100 \cdot 10^{-9}, 3300 \cdot 10^{-9}\right]$} & {$[0.3,2.7]$} & {$[0.1,7.9]$} & {$\left[100 \cdot 10^{-9}, 6500 \cdot 10^{-9}\right]$} & {$[0.3,2.7]$} \\
\hline
\end{tabular}

